\title{
On the Spectral Evolution of Cool, Helium-Atmosphere White Dwarfs: Detailed Spectroscopic and Photometric Analysis of DZ Stars
}

\author{
P. Dufour ${ }^{1}$, P. Bergeron ${ }^{1}$, James Liebert ${ }^{2}$, H. C. Harris ${ }^{3}$, G.R. Knapp ${ }^{4}$, S.F. Anderson ${ }^{5}$, \\ Patrick B. Hall ${ }^{6}$, Michael A. Strauss ${ }^{4}$, Matthew J. Collinge ${ }^{4}$, and Matt C. Edwards ${ }^{4}$
}

\begin{abstract}
We present a detailed analysis of a large spectroscopic and photometric sample of DZ white dwarfs based on our latest model atmosphere calculations. We revise the atmospheric parameters of the trigonometric parallax sample of Bergeron, Leggett, \& Ruiz (12 stars) and analyze 147 new DZ white dwarfs discovered in the Sloan Digital Sky Survey. The inclusion of metals and hydrogen in our model atmosphere calculations leads to different atmospheric parameters than those derived from pure helium models. Calcium abundances are found in the range from $\log (\mathrm{Ca} / \mathrm{He})=-12$ to -8 . We also find that fits of the coolest objects show peculiarities, suggesting that our physical models may not correctly describe the conditions of high atmospheric pressure encountered in the coolest DZ stars. We find that the mean mass of the 11 DZ stars with trigonometric parallaxes, $\langle M\rangle=0.63 M_{\odot}$, is significantly lower than that obtained from pure helium models, $\langle M\rangle=0.78 M_{\odot}$, and in much better agreement with the mean mass of other types of white dwarfs. We determine hydrogen abundances for $27 \%$ of the DZ stars in our sample, while only upper limits are obtained for objects with low signal-to-noise ratio spectroscopic data. We confirm with a high level of
\end{abstract}

\footnotetext{
${ }^{1}$ Département de Physique, Université de Montréal, C.P. 6128, Succ. Centre-Ville, Montréal, Québec, Canada H3C 3J7; dufourpa@astro.umontreal.ca, bergeron@astro.umontreal.ca

${ }^{2}$ Steward Observatory, University of Arizona, 933 North Cherry Avenue, Tucson, AZ 85721; liebert@as.arizona.edu

${ }^{3}$ US Naval Observatory, P.O. Box 1149, Flagstaff, AZ 86002-1149; hch@nofs.navy.mil

${ }^{4}$ Princeton Univ. Obs., Peyton Hall,Princeton, NJ 08544; gk@astro.princeton.edu

${ }^{5}$ Department of Astronomy, University of Washington, Box 351580, Seattle, WA 98195; anderson@astro.washington.edu

${ }^{6}$ Department of Physics and Astronomy, York University, 128 Petrie Science and Engineering Building, 4700 Keele Street, Toronto, ON M3J 1P3, Canada
} 
confidence that the accretion rate of hydrogen is at least two orders of magnitude smaller than that of metals (and up to five in some cases) to be compatible with the observations. We find a correlation between the hydrogen abundance and the effective temperature, suggesting for the first time empirical evidence of a lower temperature boundary for the hydrogen screening mechanism. Finally, we speculate on the possibility that the DZA white dwarfs could be the result of the convective mixing of thin hydrogen-rich atmospheres with the underlying helium convection zone.

Subject headings: stars: abundances - stars: atmospheres - stars: evolution white dwarfs

\section{INTRODUCTION}

Cool helium-rich white dwarfs showing traces of heavy elements (other than carbon) in their optical spectra are collectively known as DZ stars (stars with $T_{\text {eff }} \geq 12,000 \mathrm{~K}$ show He I lines and are thus classified DBZ stars). They are easily recognized by the presence of the strong resonance $\mathrm{Ca}$ II H \& K doublet in the optical and sometimes Ca I $\lambda 4226, \mathrm{Mg}$ I $\lambda 3835$ or Fe I $\lambda 3730$ (see Sion et al. 1990a, Wesemael et al. 1993, and Harris et al. 2003 for representative spectra).

Analyses of DZ white dwarfs were pioneered by Weidemann (1960), Wegner (1972), and Grenfell (1974), who were the first to analyze the classical DZ stars vMa2, Ross 640, and L745-46A using model atmosphere calculations to reproduce the optical spectrum. Other important analyses of DZ stars relying on optical data only include those of Liebert (1977), Wehrse \& Liebert (1980), Kapranidis \& Liebert (1986), Liebert et al. (1987), Sion et al. (1990b), and Dufour et al. (2006). Further progress in our understanding of these stars has also been achieved from ultraviolet observations with the International Ultraviolet Explorer (IUE) by Cottrell \& Greenstein (1980a), Cottrell \& Greenstein (1980b), Zeidler-K.T. et al. (1986), Weidemann \& Koester (1989), and Koester et al. (1990), and with the Hubble Space Telescope (HST) by Koester \& Wolff (2000) and Wolff et al. (2002). The UV region of the energy distribution is most useful for a detailed abundance analysis of elements with no strong resonance lines in the optical, and thus most of our current knowledge of DZ stars comes from UV observations.

Since the heavy elements present in the atmospheric regions sink below the photosphere in a timescale much shorter than the white dwarf cooling time (Paquette et al. 1986), the presence of metals in DZ stars must be explained in terms of episodic accretion from the 
interstellar medium, a model put on a more quantitative basis in a series of papers by Dupuis et al. (1992, 1993a, b). Following the discussion by Koester \& Wolff (2000), the most notable problem with this scenario is the low hydrogen abundances observed in these objects. Being the lightest element, hydrogen should only accumulate in the atmospheric regions of the star over time, but the observed abundances (or limits in some cases) indicate that the hydrogen accretion rate must be at least two orders of magnitude lower than that of metals (Wolff et al. 2002; Dupuis et al. 1993b). This conclusion is based on a relatively small sample of DBZ/DZ stars, albeit this was the largest homogeneous data set available at that time, and the analysis of a larger sample is required to advance our understanding of the accretion problem in cool DZ stars. Such a large sample has recently become available, thanks to the discovery of hundreds of new DZ white dwarfs by the Sloan Digital Sky Survey (SDSS, York et al. 2000; see Harris et al. 2003, for typical SDSS DZ spectra). Since these spectra cover only the optical range, their analysis will be restricted to the determination of the calcium and hydrogen abundances, since UV observations of these faint $(17 \leq g \leq 21)$ SDSS stars will not be possible in the near future. Nevertheless, the addition of these new objects will allow a clear picture to be drawn of the chemical evolution of white dwarf stars and to improve the statistical significance of earlier results since, to our knowledge, only 17 DZ stars with both $T_{\text {eff }}$ and $\mathrm{Ca} / \mathrm{He}$ measurements are found in the literature.

One of the goals of the theory of spectral evolution is to explain quantitatively the chemical evolution of cool white dwarfs. For instance, the ratio of hydrogen- to helium-dominated atmospheres is observed to vary significantly over the white dwarf cooling sequence, indicating the existence of a physical mechanism to convert one type into another. Moreover, the spectroscopic features observed in helium-rich white dwarfs vary quite significantly with temperature from the DB spectral type to the cooler DQ, DC, and DZ types. The exact reason for this evolution into either of these types is still not fully understood. To complicate the picture even further, in a fraction of cool DA stars, the upper hydrogen layers is expected to convectively mix with the underlying helium layers to form helium-rich atmosphere white dwarf stars.

Recently, Bergeron, Ruiz, \& Leggett (1997) and Bergeron, Leggett, \& Ruiz (2001, hereafter referred to as BRL97 and BLR01, respectively) analyzed the energy distributions of a large sample of cool white dwarfs with the aim of improving our understanding of their chemical evolution. Among their sample, we find several DZ stars that were analyzed under the assumption of pure helium compositions, the only models available at that time. Dufour et al. (2005) showed that the analysis of the energy distribution of DQ stars based on pure helium models overestimates the effective temperature compared to that obtained from models including carbon. This effect is due to an increase of the $\mathrm{He}^{-}$free-free opacity resulting from the additional free electrons from carbon. A similar effect is thus expected in 
the case of DZ white dwarfs. In this paper, we first include explicitly the presence of metals in our model atmosphere calculations and reanalyze the available photometric and spectroscopic data of DZ stars from BRL97 and BLR01. We then present a similar analysis of a much larger sample of DZ stars discovered in the SDSS (Gunn et al. 1998, 2006; Pier et al. 2003; Tucker et al. 2006; Stoughton et al. 2002; Abazajian et al. 2003; Adelman-McCarthy et al. 2006). In $\S 2$, we describe the observations. Our theoretical framework including our model atmosphere and synthetic spectrum calculations are presented in $\S 3$. The detailed analysis follows in $\S$ 4, and the results are interpreted and discussed in $\S$. Our conclusions are summarized in $\S 6$.

\section{OBSERVATIONS}

The first sample used for this study is drawn from the BRL97 and BLR01 analyses, which include $12 \mathrm{DZ}$ stars with optical $B V R I$ and infrared $J H K$ photometry as well as trigonometric parallax measurements, with the exception of ESO 445-271 (WD 1338-311), for which the parallax and $J H K$ measurements are not available. The photometric data can be found in Tables 1 of BRL97 and BLR01. Our analysis also relies on spectroscopic observations taken from various sources. New high signal-to-noise ratio $(\mathrm{S} / \mathrm{N})$ spectroscopic observations at a resolution of $\sim 6 \AA \mathrm{FWHM}$, covering the Ca II H \& K doublet region have been secured with the Mont Mégantic Observatory $1.6 \mathrm{~m}$ telescope in 2004 September (WD 0046+051, WD 0802+386, WD 1626-368, and WD 1705+030) and with the Steward Observatory $2.3 \mathrm{~m}$ telescope in 2004 May (WD 2251-070 and WD 2312-024). Optical spectra for the remaining stars were secured by BRL97 and BLR01 (details of the observations are provided in these references).

Our second sample consists of DZ white dwarfs spectroscopically identified in the SDSS. We selected all DZ stars from the First and Fourth Data Release white dwarf catalogs (Kleinman et al. 2004; Eisenstein et al. 2006). These catalogs are not complete in any way (see below) and several more DZ white dwarfs are certainly present in the SDSS archive. Additional DZ stars have also been added to our list as they were discovered serendipitously by examination of the SDSS spectroscopic archive. Our final sample consists of 147 SDSS DZ stars with spectra covering the 3800-9200 $\AA$ region at a resolution of $\sim 3 \AA$ FWHM. Also available are SDSS photometric observations on the ugriz system (Fukugita et al. 1996; Hogg et al. 2001; Smith et al. 2002; Ivezić et al. 2004). Combining the two data sets, we have a large sample of 159 DZ white dwarfs that can be analyzed in a homogeneous fashion. This is a considerably larger sample than the $17 \mathrm{DZ}$ stars with both $T_{\text {eff }}$ and Ca/He determinations analyzed by various groups in the last $\sim 50$ years or so. 


\section{MODEL ATMOSPHERE AND SYNTHETIC SPECTRUM CALCULATIONS}

Our LTE model atmosphere code is similar to that described in Dufour et al. (2006) for the study of the DZ white dwarf G165-7. It is based on a modified version of the code described at length in Bergeron et al. (1995), which is appropriate for pure hydrogen and pure helium atmospheric compositions, as well as mixed hydrogen and helium compositions, while energy transport by convection is treated within the mixing-length theory. One important modification is that metals and molecules are now included in the equation-of-state and opacity calculations (see Dufour et al. 2005, 2006, for details). As was the case for DQ stars, $\mathrm{He}^{-}$free-free absorption is found to be the dominant source of opacity in DZ stars. It is thus important to account for all possible sources of electrons in the equation-of-state, and we have included here all elements with $Z \leq 26$. The chemical abundances cannot be determined individually, however, since most of these elements are not observed spectroscopically. We thus initially assume that the relative abundances are consistent with solar ratios; this is a reasonable assumption, at least for the observed elements, according to our analysis of G1657 (Dufour et al. 2006) and that of Wolff et al. (2002) for several DZ stars (see their Fig. 7). We show below that this assumption is not very critical for our atmospheric parameter determinations.

More critical is the assumed hydrogen abundance for stars not showing $\mathrm{H} \alpha$ absorption. To explore this issue, we calculated 4 grids with respectively $\log (\mathrm{H} / \mathrm{He})=-3,-4,-5$, and -30 respectively. Our model grids cover a range of atmospheric parameters from $T_{\text {eff }}=4000$ to $12,000 \mathrm{~K}$ in steps of $500 \mathrm{~K}$ at a fixed value of $\log g=8$, and $\log (\mathrm{Ca} / \mathrm{He})=-12$ to -7 in steps of 0.5 dex, while the relative abundances of all elements heavier than helium are set with respect to the calcium abundance in solar ratios. These assumptions will need to be verified a posteriori. We also calculated a smaller grid with $\log (\mathrm{H} / \mathrm{He})=-6, T_{\text {eff }}=9000$ to $12,000 \mathrm{~K}$, and $\log (\mathrm{Ca} / \mathrm{He})=-11$ to -8 (with identical steps as above). In order to assess the influence of the Ly $\alpha$ line wing opacity, we calculated an additional grid with $\log (\mathrm{H} / \mathrm{He})=-3$ including the Ly $\alpha$ profile calculations described in Koester \& Wolff (2000), kindly made available to us by D. Koester (2005, private communication). To take into account, in an approximate fashion, the non-ideal effects on heavy elements, we also calculated models at $\log (\mathrm{H} / \mathrm{He})=-5$ with the occupation probability formalism of Hummer \& Mihalas (1988). This formalism may not be adequate for stars with extremely high atmospheric pressure, however (see, e.g., Saumon et al. 1995), and it is used mainly to allow a better comparison with other models in the literature (Koester \& Wolff 2000; Wolff et al. 2002). Finally, we computed additional models with different relative abundances of metals and values of $\log g$, to explore the sensitivity of our results to these assumptions. 
In addition to the increased $\mathrm{He}^{-}$free-free continuum opacity, important metal absorption features in the ultraviolet may potentially affect the energy distributions and thus the atmospheric structures compared to those obtained from pure helium models. Thus, over 4000 of the strongest metal lines $-\sim 2600$ lines from Fe I alone - are included explicitly in both the model and synthetic spectrum calculations. These lines are selected by taking all lines contributing more than one tenth of the $\mathrm{He}^{-}$free-free opacity in the range $\tau_{R}=0.1-1.0$ from several models at $\log \mathrm{Ca} / \mathrm{He}=-7$ and $T_{\text {eff }}$ between 5000 and 12,000 $\mathrm{K}$. We are confident that this line list includes all the important contributors to the atomic opacity, since spectra calculated by increasing the number of lines by an order of magnitude did not have any detectable effect on the emergent spectrum. The line absorption coefficient is calculated using a Voigt profile for every line at every depth point. The line broadening is treated within the impact approximation with van der Waals broadening by neutral helium. Central wavelengths of the transitions, $g f$ values, energy levels, and damping constants are extracted from the GFALL line list of R. L. Kuruc21.

Illustrative spectra from our model grid at $\log g=8$ are displayed in Figure 1 for various values of the effective temperature, metal, and hydrogen abundances. We first notice that large hydrogen abundances of $\log (\mathrm{H} / \mathrm{He}) \sim-3$ lead to a reduction of the width and depth of the Ca II H \& K doublet compared to hydrogen-free models. This can be explained in terms of the increased $\mathrm{He}^{-}$free-free opacity produced by the free electrons coming from hydrogen when its abundance is sufficiently large, which actually outnumber the contribution from metals. Since the pressure gradient is inversely proportional to the Rosseland mean opacity, an increase in the opacity results in a drop of the atmospheric pressure and corresponding pressure broadening of the atomic lines. The competition between metals and hydrogen as electron donors is illustrated in Figure 1 by comparing spectra at $T_{\text {eff }}=9000 \mathrm{~K}$ with $\log (\mathrm{Ca} / \mathrm{He})=-8.0$ and -10.0 . The effect of the presence of hydrogen in the latter case can already be observed at $\log (\mathrm{H} / \mathrm{He})=-5$, while the contribution of hydrogen can only be seen at much larger abundances in the $\log (\mathrm{Ca} / \mathrm{He})=-8.0$ model. At 12,000 K, the effect is less pronounced since the contribution from helium to the free electrons becomes significant, and models with $\log (\mathrm{H} / \mathrm{He})=-5$ are practically identical to those without hydrogen. At $\log (\mathrm{H} / \mathrm{He})=-3$, hydrogen becomes the principal electron donor at 12,000 $\mathrm{K}$ (note also the presence of $\mathrm{H} \delta$ at $4101 \AA$ ). Of course, if one is interested in the determination of the hydrogen abundance in this temperature range, direct observations at $\mathrm{H} \alpha$ are certainly more useful since this line can be detected for hydrogen abundances as low as $\log (\mathrm{H} / \mathrm{He}) \sim-5$ at $T_{\text {eff }}=10,000 \mathrm{~K}$ (not shown here).

\footnotetext{
${ }^{1}$ see http://kurucz.harvard.edu/LINELISTS.html
} 
At lower effective temperatures, the hydrogen abundance can be directly determined through $\mathrm{H} \alpha$ only when the line is spectroscopically visible, which occurs at relatively large abundances - approximately $\log (\mathrm{H} / \mathrm{He}) \sim-3$ at $6500 \mathrm{~K}$ and $\log (\mathrm{H} / \mathrm{He}) \sim-6$ near 10,000 $\mathrm{K}$. However, in the intermediate temperature range between $T_{\text {eff }}=6000$ and $8500 \mathrm{~K}$, the hydrogen abundance can be determined indirectly from observations of the core depth and wing profiles of the $\mathrm{Ca}$ II $\mathrm{H} \& \mathrm{~K}$ doublet, as demonstrated with the $7500 \mathrm{~K}$ spectra shown in Figure 1. At lower temperatures, the effect of hydrogen on the synthetic spectrum becomes less important since the low ionization potential of metals relative to hydrogen favors the contribution of free electrons from metals rather than hydrogen. Finally, metallic absorption features become very strong at low effective temperatures $\left(T_{\text {eff }} \sim 6000 \mathrm{~K}\right)$ for abundances typical of those found in hotter DZ stars, $\log (\mathrm{Ca} / \mathrm{He}) \sim-8.0$, producing objects similar to the unique DZ star G165-7. Also at low effective temperatures, collision induced absorption (CIA) by molecular hydrogen quickly dominates the infrared opacity, providing a severe constraint on the presence of hydrogen (see below).

\section{DETAILED ANALYSIS}

\subsection{Atmospheric Parameter Determination}

The method used to fit the photometric and spectroscopic data is similar to that described at length in Dufour et al. (2005) and Dufour et al. (2006). Briefly, we first estimate the effective temperature of the star by fitting the global energy distribution as provided by the $B V R I$ and $J H K$ (or ugriz for SDSS stars) photometric observations. The fitting procedure relies on the nonlinear least-squares method of Levenberg-Marquardt (Press et al. 1992). Here, both $T_{\text {eff }}$ and the solid angle $\pi(R / D)^{2}$, which relates the flux at the surface of the star to that received at Earth, are considered free parameters ( $R$ is the radius of the star and $D$ its distance from Earth). Since the temperature obtained from the energy distribution depends on the assumed chemical composition, we use the spectroscopic observations to constrain the metal abundances. We thus assume the $T_{\text {eff }}$ value obtained from the photometry and determine the chemical composition by fitting the Ca II H \& K doublet region with our grid of synthetic spectra. We also measure the hydrogen abundance by fitting the $\mathrm{H} \alpha$ absorption line if present; otherwise, we proceed at a fixed value of log $(\mathrm{H} / \mathrm{He})$ (see below). A new estimate of the effective temperature is then obtained by fitting the photometric observations with models interpolated at the metal and hydrogen abundances determined from the spectroscopic fit (all metal abundances are assumed solar with respect to calcium). The

procedure is then iterated until the atmospheric parameters have converged to a consistent photometric and spectroscopic solution. 
For 11 DZ stars with known distances obtained from trigonometric parallax measurements, we can obtain the radius of the star from the solid angle from our modelling, which in turn can be converted into $\log g$ (or mass) using evolutionary models similar to those described in Fontaine et al. (2001) but with C/O cores, $q(\mathrm{He}) \equiv \log M_{\mathrm{He}} / M_{\star}=10^{-2}$, and $q(\mathrm{H})=10^{-10}$, which are representative of helium-atmosphere white dwarf 2 .

\subsection{Reappraisal of the BRL97 and BLR01 analyses}

We begin by re-evaluating the atmospheric parameters of the 12 DZ stars in the BRL97 and BLR01 samples using our new models that incorporate metals. In addition to the contribution of metals as electron donors in the atmospheres of DZ stars, the contribution from hydrogen also needs to be properly evaluated. Hydrogen abundances can be determined directly from spectroscopic observations for only three stars in our sample, namely Ross 640, L745-46A, and G165-7, which explicitly show $\mathrm{H} \alpha$. For the remaining DZ stars, upper limits on the hydrogen abundance can be obtained from the absence of a detectable $\mathrm{H} \alpha$ feature, or for the coolest stars, from the infrared energy distribution, since the CIA opacity becomes particularly important in a mixed $\mathrm{H} / \mathrm{He}$ atmosphere due to the collisions of $\mathrm{H}_{2}$ with neutral helium. The assumed hydrogen abundance for the analysis of DZ stars is more important than it was for DQ stars (see Dufour et al. 2005) because the high carbon abundances (relative to hydrogen) in DQ stars make hydrogen a minor contributor to the total electron population. The uncertainties of our atmospheric parameter determination introduced by the use of models with various hydrogen abundances is discussed at length below.

For DZ stars showing $\mathrm{H} \alpha$ (the DZA stars), we use the observed line profile to determine $\log (\mathrm{H} / \mathrm{He}), \log (\mathrm{Ca} / \mathrm{He})$, and $T_{\text {eff }}$ in a consistent manner as described above. When $\mathrm{H} \alpha$ is not detected spectroscopically, we fit each star with $\log (\mathrm{H} / \mathrm{He})$ fixed at $-30,-5,-4$, and -3 , and carefully examine each solution and reject those that predict a detectable $\mathrm{H} \alpha$ absorption feature given the $\mathrm{S} / \mathrm{N}$ of each spectrum. We also reject solutions that predict an infrared flux deficiency due to the CIA opacity that is incompatible with the observed photometry at $J H K$. As discussed above (see Fig. 1), we find some cases where the Ca II $\mathrm{H} \& \mathrm{~K}$ lines (core and/or wings) are better reproduced with models including a trace of hydrogen. This is a direct consequence of the increased opacity (more free electrons from hydrogen), which reduces the atmospheric pressure, producing narrower line profiles. Note that the surface gravity may also affect the atmospheric pressure, but models calculated at $\log g=8.0 \pm 0.25$ reveal that the Ca II H \& K line wings are not as strongly affected.

\footnotetext{
${ }^{2}$ see http://www.astro.umontreal.ca/ bergeron/CoolingModels/
} 
Figure 2 and 3 illustrate examples of solutions with various hydrogen abundances for the DZ stars WD 1705+030 and WD 2312-024, where we can clearly see that the solutions with $\log (\mathrm{H} / \mathrm{He})=-3$ and -4 , respectively, provide a better match to the observed calcium lines than do models without hydrogen. Such indirect hydrogen abundance determinations are inherently more uncertain than a direct determination through the $\mathrm{H} \alpha$ line profile, however, since it is possible for elements spectroscopically invisible to contribute to the number of free electrons in a significant way. Thus for those particular stars with no detectable $\mathrm{H} \alpha$, we obtain new atmospheric parameters by fitting explicitly the Ca II H \& K profiles, but this time by considering the hydrogen abundance as a free parameter in our fitting procedure.

We present in Table 1 our atmospheric parameter determinations (effective temperature, surface gravity, stellar mass, and calcium abundance) for the BRL97 and BLR01 samples. Quantities in parentheses represent the formal $1 \sigma$ uncertainties of each parameter obtained from our fitting procedure, with the exception of $\log g$ and the mass, for which the uncertainties are obtained by propagating the error of the trigonometric parallax measurement. Note that these formal errors are relatively small for some $\log (\mathrm{Ca} / \mathrm{He})$ and $\log (\mathrm{H} / \mathrm{He})$ values, and this certainly does not reflect the true uncertainties. Indeed, the values given here represent only the formal internal uncertainties of the fitted atmospheric parameters obtained from the covariance matrix (see Press et al. 1992 for details). We find, from visual inspection, that changing the abundance by about 0.2 dex still gives adequate fits. We thus estimate instead that an uncertainty of \pm 0.2 dex is probably more realistic (except for the coolest stars, see below). A similar conclusion was reached by Provencal et al. (2002, see their Fig. 6) in the case of Procyon B. To bracket the possible range of solutions for stars whose hydrogen abundance could not be determined directly or indirectly, we provide two solutions in Table 1 , one with the maximum amount of hydrogen allowed by the photometric and spectroscopic observations, and the other solution without any hydrogen ("no H" in Table 1). However, Wolff et al. (2002) found, on the basis of their analysis of UV observations, that hydrogen is present in virtually all white dwarfs in their sample. We thus believe that one should not artificially separate the DZ stars showing traces of hydrogen (the DZA stars) from those showing no hydrogen features, since this distinction is mostly due to the level of visibility of hydrogen and the $\mathrm{S} / \mathrm{N}$ of the spectra. Hence, our solutions that include hydrogen in Table 1 are probably more realistic.

The corresponding fits to the $B V R I$ and $J H K$ photometric observations and calcium lines are displayed in Figures 4 and 5, To our knowledge, the calcium abundances for the DZ stars WD 0552-041 (G99-44), WD 1313-198 (LHS 2710), WD 1338-311 (ESO 445-271), WD 1705+030 (G139-13), and WD 2345-447 (ESO 292-43) are determined here for the first time. Some stars deserve additional comments: 
WD 0046+051 (vMa 2): Our solution, $T_{\text {eff }}=6220 \mathrm{~K}, \log (\mathrm{Ca} / \mathrm{He})=-10$, and $\log (\mathrm{H} / \mathrm{He})=$ -3.19 , differs slightly from that obtained by Wolff et al. (2002), $T_{\text {eff }}=5700 \mathrm{~K}, \log (\mathrm{Ca} / \mathrm{He})=$ -10.65 , and $\log (\mathrm{H} / \mathrm{He})=-5$. Their solution requires a small trace of hydrogen in order to reproduce the IUE flux level, which is reduced significantly by their improved Ly $\alpha$ line profile calculations. Our hydrogen abundance determination, on the other hand, is obtained indirectly from the $\mathrm{Ca}$ II $\mathrm{H} \& \mathrm{~K}$ profiles. If we assume instead a value of $\log (\mathrm{H} / \mathrm{He})=$ -5 , we find $T_{\text {eff }}=6010 \mathrm{~K}, \log (\mathrm{Ca} / \mathrm{He})=-10.65$, closer to the Wolff et al. solution, although our fits to the calcium line cores and wings are not as satisfactory with this smaller hydrogen abundance. Note that Wolff et al. also include non-ideal effects using the HummerMihalas occupation probability formalism. To evaluate the effects of the different input physics between the two sets of models, we calculated a single model with the Wolff et al. atmospheric parameters by also including the Hummer-Mihalas formalism for metals and the same Ly $\alpha$ theoretical profiles. Our best fit to this single model with our original grid at $\log (\mathrm{H} / \mathrm{He})=-3$ yields $T_{\text {eff }}=6147 \mathrm{~K}$ and $\log (\mathrm{Ca} / \mathrm{He})=-10.48$, close to our original estimate of the effective temperature. In other words, had we calculated a full grid with the Hummer-Mihalas occupation probability formalism, we would probably have found atmospheric parameters close to that obtained by Wolff et al. (2002). Hence the differences between both solutions can be partially explained in terms of the small differences in the input physics.

WD 0552-041 (LP 658-2): Wolff et al. (2002) obtain a better match to Faint Object Spectrograph (FOS) observations by including a small trace of hydrogen of $\mathrm{H} / \mathrm{He}=5 \times 10^{-4}$, but they do this at $T_{\text {eff }}=5050 \mathrm{~K}$, the effective temperature obtained by BLR01 under the assumption of a pure helium composition. Metals must have an influence on both the temperature structure and UV absorptions that are not taken into account in the BLR01 solution. Our iterative procedure does indeed suggest a much lower effective temperature for this object, $T_{\text {eff }} \sim 4300 \mathrm{~K}$. Note that at this temperature, $J H K$ photometry would be affected by the collision induced opacity (not included in Wolff et al. 2002) if $\log (\mathrm{H} / \mathrm{He})$ is higher than $\sim-5$. Our metal abundance is obtained from the extremely weak Ca II H \& K lines. However, our solution also predicts a strong Ca I $\lambda 4226$ line that is not observed spectroscopically. We can only speculate at this point on the reason for this discrepancy. One possibility is that neutral calcium could be pressure ionized under the extreme conditions found in this star, although it is strange to see a strong Ca I $\lambda 4226$ feature in WD 2251-070, a DZ star with presumably even higher photospheric pressures. We were also not able to achieve an acceptable fit using Koester's Ly $\alpha$ profiles or the occupation probability formalism in our model calculations. A good fit to both sets of lines is possible only by changing the effective temperature in such a way that the photometric fit is no longer acceptable. Clearly, more effort in the modeling of high atmospheric pressure white dwarfs is required. 
WD 0738-172 (L745-46A), WD 1626+368 (Ross 640): Our solutions for these stars, $T_{\text {eff }}=$ $7590 \mathrm{~K}, \log (\mathrm{Ca} / \mathrm{He})=-10.91$ and $T_{\text {eff }}=8440 \mathrm{~K}, \log (\mathrm{Ca} / \mathrm{He})=-8.83$, respectively, are very close to those obtained by Koester \& Wolff (2000), $T_{\text {eff }}=7500 \mathrm{~K}, \log (\mathrm{Ca} / \mathrm{He})=-10.6$ and $T_{\text {eff }}=8500 \mathrm{~K}, \log (\mathrm{Ca} / \mathrm{He})=-8.65$, respectively. The latter were obtained from fits to the Ca II lines and from FOS spectra. In order to reproduce the observed fluxes in the UV, Wolff et al. had to include their improved Ly $\alpha$ theoretical calculations. Although our models do not include this opacity and fail to reproduce the UV fluxes, our atmospheric parameters do not differ significantly from those of Wolff et al. The reason for this result is that the UV flux absorbed by the Ly $\alpha$ wings represents only a small fraction of the total flux, and its redistribution to other wavelengths affects the thermodynamic structure only slightly. To test this hypothesis, we calculated several models including the Ly $\alpha$ profile and fitted the resulting synthetic spectra and energy distributions with our original grid. The differences in $T_{\text {eff }}$ and $\log (\mathrm{Ca} / \mathrm{He})$ are always smaller than $\sim 150 \mathrm{~K}$ and 0.20 dex, respectively, well within the measurement uncertainties for these objects. Given that these differences are small, we refrain from including systematically this Ly $\alpha$ profile in our model calculations since the approach used by Wolff et al. has been successfully tested for only a few stars that are relatively hot $(7500$ and $8500 \mathrm{~K})$, and it is not clear whether this formalism remains valid at lower temperatures.

WD 0802+386 (LP 257-28): This object is the hottest DZ star $\left(T_{\text {eff }}=10,980 \mathrm{~K}\right)$ in the BRL97/BLR01 sample. At this temperature, helium-rich white dwarfs are expected to show $\mathrm{H} \alpha$ even for hydrogen abundances as low as $\log (\mathrm{H} / \mathrm{He})=-5.0$. Our featureless spectrum near $\mathrm{H} \alpha$ is thus extremely surprising considering that accretion of hydrogen with a rate as low as $10^{-20} M_{\odot} \mathrm{yr}^{-1}$ should provide enough hydrogen to be easily detected spectroscopically. We postpone the discussion of this object after the analysis of the DZ stars from the SDSS, which include several white dwarfs similar to LP 257-28.

WD 1328+307 (G165-7): This star has a magnetic field and has been analyzed separately by Dufour et al. (2006). The atmospheric parameters given in Table 1 and the fit with magnetic models displayed in Figure 4 are taken from that analysis.

WD 2251-070 (LP 701-29): The best fit obtained for this star by Kapranidis \& Liebert (1986) reproduces the Ca I $\lambda 4226$ line but fails to provide an acceptable fit to the Ca II lines. The authors argue that pressure ionization probably affects the metal ionization equilibrium, but an exact treatment was not available at that time and could not be tested further. We find that the photometric and spectroscopic observations are fairly well reproduced by our hydrogen-free models; note that our temperature estimate for this star, $T_{\text {eff }}=4000 \mathrm{~K}$, is at the limit of our grid. In this temperature range, even though the $\mathrm{H} \alpha$ line is spectroscopically invisible, the hydrogen abundance can be easily constrained from the infrared JHK pho- 
tometry since the $\mathrm{H}_{2}$-He CIA opacity becomes important even for hydrogen abundances as low as $\log (\mathrm{H} / \mathrm{He})=-5$. We note also that Kapranidis \& Liebert (1986) used pure helium stratifications for their synthetic spectrum calculations. Our models reveal significant differences between the pressure and temperature structures of pure helium models and those including metals. It is unclear, however, how this would translate in the models of Kapranidis \& Liebert calculated using a different theoretical framework based on a Thomas-Fermi equation-of-state, which also includes electron thermal conduction as an energy transfer mechanism. Although not perfect, our best fit reproduces the neutral calcium line well, and shows a hint of ionized calcium. Our models fail, however, to reproduce the metallic blend near $4500 \AA$. It is clearly inconsistent to invoke pressure ionization to explain the absence of Ca I $\lambda 4226$ in WD 0552-041 when the same line is very strong in a star characterized with even higher atmospheric pressures. This result suggests that the physics of cool helium-rich models at high densities is not fully understood yet, and that significant improvements are still required.

The mean mass for the $11 \mathrm{DZ}$ stars with trigonometric parallax measurements analyzed in this paper is $\langle M\rangle=0.63 M_{\odot}$. As discussed above, however, the atmospheric parameters of the five coolest stars are certainly more uncertain than those of the rest of the sample. If we exclude these objects, the mean mass rise slightly to $\langle M\rangle=0.66 M_{\odot}$, significantly lower than the average mass obtained by BRL97/BLR01, $\langle M\rangle=0.78$ (or $\langle M\rangle=0.82 M_{\odot}$ if we exclude L745-46A and Ross 640 which were analyzed with mixed H/He models by BRL97 and BLR01). This difference in average mass can be readily explained in terms of the free electrons provided by the metals as well as hydrogen, which both increase the opacity in our model calculations.

Beauchamp (1995) found that the mass distribution of DBA stars significantly differs from that of DB stars, with a higher fraction of massive white dwarfs above $M \sim 0.65 M_{\odot}$. Moreover, the three most massive stars in their sample were DBA stars, suggesting a possible link between the presence of hydrogen and the mass of the star. We do not find such a trend in our sample of DZ stars, the possible descendents of DB white dwarfs. In fact, one of the most massive stars in our sample, WD $0802+386$, has an extremely small upper limit on its hydrogen content. However, considering the limited size of our sample, we remain cautious and will refrain from drawing any definitive conclusion on the interpretation of the mass distribution of $\mathrm{DZ}$ white dwarfs. 


\subsection{Analysis of DZ Stars from the SDSS}

Prior to this work, abundance analyses of DZ white dwarfs $\left(T_{\text {eff }}<12,000 \mathrm{~K}\right)$ had been carried out for only 17 stars. Our SDSS sample alone contains 147 new DZ stars. These additional objects discovered in the SDSS allow a significant improvement in the statistics of DZ stars. The method we adopt to fit the SDSS data is similar to that described above, with the exception that the SDSS ugriz photometry is used instead of the $B V R I$ measurements. These photometric bands cover the entire optical range from the UV atmospheric cutoff $(3200 \AA)$ to the red sensitivity cutoff of the detector $(\sim 10,000 \AA)$. Furthermore, since trigonometric parallax measurements are not available for the SDSS stars, we assume a value of $\log g=8.0$ for all objects. As was the case for the BRL97 and BLR01 samples, the hydrogen abundance for most stars is unknown. We thus fit all stars with our model grids with various hydrogen abundances, and then reject the solutions that are incompatible with the observations at $\mathrm{H} \alpha$, or determine directly the hydrogen abundances when $\mathrm{H} \alpha$ is visible, or indirectly through the Ca II H \& K line profiles whenever possible (i.e. for spectra with good enough $\mathrm{S} / \mathrm{N}$ ratio).

Our adopted atmospheric parameters are presented in Table 2. We have not applied any correction for Galactic extinction since the stars are relatively close, therefore only a small fraction of the absorption should be applied (see Dufour et al. 2005, for more on this issue). Figures 6 to 24 show our best fits to the energy distribution, to the Ca II H \& K lines, and to the $\mathrm{H} \alpha$ region for the maximum hydrogen abundance allowed by the spectroscopic observations, or for the hydrogen abundance obtained from fits to $\mathrm{H} \alpha$ when it is visible spectroscopically.

There is another set of Ca II lines available in the red portion of the SDSS spectra at 8498, 8542, and 8662 А (the "infrared triplet") that can be used as an internal consistency check. However, these lines are not as strong as the $\mathrm{H} \& \mathrm{~K}$ lines, and they are detected only at high calcium abundances above $\log (\mathrm{Ca} / \mathrm{He})>-9$ at the $\mathrm{S} / \mathrm{N}$ typical of our spectra. Our models predict the presence of these lines in several objects, but a direct comparison could only be achieved for stars with good signal-to-noise ratio spectra. Figure 25 shows the DZ stars with recognizable Ca II lines together with our synthetic spectra interpolated at the atmospheric parameter solution obtained from the $\mathrm{Ca}$ II $\mathrm{H} \& \mathrm{~K}$ lines. We find a good internal consistency between the two sets of lines for three out of four stars with sufficiently high $\mathrm{S} / \mathrm{N}$ to allow a useful comparison (the bottom four objects). Higher $\mathrm{S} / \mathrm{N}$ spectroscopic observations would be required for the other DZ stars in our sample. For SDSS J103809.19-003622.5, we observe a discrepancy between both sets of lines, the Ca II lines in the red favoring a higher effective temperature than that inferred from the photometry (our photometric and spectroscopic fits shown in Fig. 15] are not very good for this object). 
One possible solution is that this star is an unresolved degenerate binary. We also find one peculiar object (SDSS J155852.59+031242.9) showing strong absorption features at the position of the Ca II lines that are at odds with the predicted profiles.

The calcium lines displayed in Figure 25] are also more sensitive to the presence of a weak magnetic field since the separation of the Zeeman components is proportional to the square of the central wavelength of the line, $\Delta \lambda=9.34 \times 10^{-13} \lambda_{c}^{2} g_{\text {eff }} B_{s}$ (see for instance the analysis of G165-7 by Dufour et al. 2006). The absence of any line splitting translates into an upper limit of $\sim 150 \mathrm{kG}$ for the four stars shown in Figure 25. Higher S/N spectroscopic observations of the other stars in our sample could potentially provide interesting limits on the presence of magnetic fields in DZ white dwarfs.

\subsection{Comments on the Assumed Solar Composition}

Our synthetic spectrum calculations assume that the relative abundance of heavy elements is solar; the metal content is thus fixed by our fit to the calcium lines. Is this a reasonable assumption? Most heavy elements are not observed in the optical spectrum and thus there is no way to determine individual abundances. As for the elements that are visible only occasionally (e.g., magnesium, iron), detailed abundance analyses in the optical are extremely limited, and one has to rely on UV observations to obtain a reasonable estimate of the metal content. Unfortunately, UV observations are available only for a small number of DZ stars, the most complete analysis being that of Wolff et al. (2002). According to their Figure 7, most cool stars exhibit metal-to-metal ratios that are compatible with solar ratios within 1 dex. Our calculations indicate that small variations of metal abundances (other than calcium) with respect to solar ratios do not have any significant effect on our atmo-

spheric parameter determinations. It thus seems reasonable to assume that the metals are present in the atmospheres of DZ stars in solar ratios, or at least not too far from solar. Also, models calculated with the abundances of all elements never observed in DZ stars set to zero are practically identical to those calculated with solar values with respect to calcium. This is not surprising in view of the fact that hydrogen, magnesium, and iron are always the principal electron donors.

Our SDSS sample contains only a few objects showing elements other than calcium in their optical spectrum (see, e.g., the Mg and Fe lines near $3850 \AA$ in Figs. 6] to 24). Although the $\mathrm{S} / \mathrm{N}$ ratio is relatively low, the predicted and observed metallic features for these few stars are at least compatible with solar ratios. The DZ star SDSS J095645.15+591240.6 shown in Figures 13 (blue part) and 26 (red part) represents an extreme example of a DZ star with metal abundances consistent with solar ratios based on an examination of the various 
iron and magnesium lines. Also displayed in Figure 26 are white dwarf stars that exhibit the Mg I "b" blend at $\sim 5175 \AA$. The feature is strongest in SDSS J095645.15+591240.6, where the famous asymmetry of the blue wing can clearly be seen. Dufour et al. (2006) argued that the asymmetry in the magnetic DZ star G165-7 was possibly due to molecular absorption by $\mathrm{MgH}$. However, at $T_{\text {eff }}=8230 \mathrm{~K}$, SDSS J095645.15+591240.6 is too hot for this molecule to be visible, and another explanation must be sought. Note that an increase of the magnesium abundance would produce lines in the $3800 \AA$ region that would be incompatible with the observations. SDSS J103809.19-003622.5, discussed in the previous section, is also problematic here.

A metal-to-metal ratio analysis similar to those of Dupuis et al. (1993b) and Wolff et al. (2002) is not possible here since our sample contains only a few objects with more than one heavy element observed. However, our study can be used to identify the best candidates for future UV observations (for abundance analyses) and IR observations (to find possible debris disks or dust clouds around white dwarfs; see similar studies around DAZ stars in Reach et al. 2005; Kilic et al. 2005; Becklin et al. 2005; Kilic et al. 2006). In conclusion, models calculated with solar abundance ratios reproduce all features observed in the optical (with the exception of the Mg I "b" blend), and they do not predict absorption features that are not observed.

\section{Results}

\subsection{Calcium Abundances}

Our results are summarized in Figure 27 where the calcium abundance for all DZ stars analyzed in this paper are shown as a function of effective temperature. The various symbols are described in the figure caption. The two continuous curves correspond to the predicted equilibrium abundances reached in the low and high phases of the two-phase accretion scenario proposed by Dupuis et al. (1993b), which correspond to accretion rates of $5 \times 10^{-20}$ and $5 \times 10^{-15} M_{\odot} \mathrm{yr}^{-1}$, respectively. Whether the origin of metals is related to encounters with interstellar clouds or attributed to comets or asteroids, it is comforting to find that the accretion rates required to explain the calcium abundances in DZ stars are about the

same as for the DAZ stars (Koester \& Wilken 2006), a result that suggests that the metals observed in DZ and DAZ stars have the same origin.

We first notice that the atmospheric parameters of our DZ stars fill most of the $\mathrm{Ca} / \mathrm{He}$ - $T_{\text {eff }}$ plane between the two continuous curves, with the exception of some regions discussed here. First and most obvious is the empty triangular region at high effective temperatures 
and low calcium abundances. No stars are found in this region simply because the Ca II H \& $\mathrm{K}$ transitions are not sufficiently excited to be detected spectroscopically. Stars in this particular region would thus appear as DC white dwarfs, and only ultraviolet observations would allow one to fill this part of the plane. The dashed line represents the detection threshold for the Ca II H \& K lines calculated for a limit set at $5 \AA$ of total equivalent width (a typical value for calcium lines observed in low $\mathrm{S} / \mathrm{N}$ spectra).

We also find a region near $\sim 6500 \mathrm{~K}$ and high calcium abundances where the number of stars is significantly reduced (the only star in this region is G165-7 discussed below). Figure 28 shows that cool DZ stars form a parabola in the $(g-r, u-g)$ color-color diagram at a given effective temperature, and that models with high calcium abundances overlap the stellar locus region (highest density point region). Objects in the SDSS are selected for follow-up spectroscopic observations mainly on the basis of their colors. High priority is given to potential QSOs that have colors outside the stellar locus (Richards et al. 2002). The paucity of stars in the regions of the $\mathrm{Ca} / \mathrm{He}-T_{\text {eff }}$ plane discussed above can thus be explained in part as a selection effect in the SDSS targeting procedure. The absence of cool stars can also be explained by the fact that most of our stars come from the DR4 catalog of Eisenstein et al. (2006). As shown by their Figure 1, a color cut was made to eliminate objects close or in the stellar locus before they search for white dwarfs candidates. Cool DZ stars in these regions, if they exist, should be found by a more careful search of the SDSS spectroscopic archive, or with the help of reduced proper motion surveys similar to that undertaken by Kilic et al. (2006).

The only star in the upper-right corner of Figure 27 is G165-7. However, the low effective temperature and high metallic content of G165-7 produce strong absorption features and unusual colors that put it on the other side of the stellar locus, far to the right and outside the color range displayed in Figure28. Note that the parameters given in Table 1 suggest that G165-7 would be near the stellar locus. However, these parameters were obtained partially with $B V R I$ and $J H K$ photometry, and the fit to $u$ and $g$ in Figure 6 of Dufour et al. (2006) is not very good.

Finally, we find no DZ stars between $T_{\text {eff }} \sim 5000$ and $6000 \mathrm{~K}$. Note that vMa 2 should probably be in that temperature range according to the analysis of Wolff et al. (2002) based on models calculated with a non-ideal equation of state. As explained above, such cool stars have colors that overlap the stellar locus, and it is therefore not surprising to not find any DZ star in this particular range of temperature in the SDSS sample. There is also no DZ star in the BRL97 and BLR01 samples in the same temperature range. However, a significant deficiency of helium atmosphere white dwarfs has been observed, the so-called non DA gap, in the $(V-I, V-K)$ color-color diagram shown in Figure 9 of BLR01. But since our sample 
contains only a few DZ stars in the vicinity of the gap, and since the physics in this high pressure regime is not fully understood, the absence of DZ stars between $\sim 5000$ and $6000 \mathrm{~K}$ is not statistically significant, and no firm conclusion concerning the nature of the gap can be reached from our results.

\subsection{Hydrogen Abundances}

Our SDSS sample contains 27 objects with a detectable $\mathrm{H} \alpha$ feature, while we achieve a better fit to the $\mathrm{Ca}$ II $\mathrm{H} \& \mathrm{~K}$ lines for 10 additional objects if hydrogen is included, for a total of $37 / 147$ stars (or $\sim 25 \%$ ) with hydrogen detected directly or indirectly. The SDSS sample is nowhere complete in volume or magnitude, but since SDSS white dwarfs are selected randomly and independently of the spectral type, stars with hydrogen should not be preferentially chosen, so we believe that this ratio is representative of the entire DZ population. For the BRL97 and BLR01 samples, there are three stars showing $\mathrm{H} \alpha$, and three more for which the hydrogen abundance could be determined indirectly. We thus have a total of 43/159 stars (or 27\%) with hydrogen abundances determined in our entire sample.

Figure 29 shows our hydrogen abundance determinations (or upper limits) as a function of effective temperature for our full sample. Also indicated are the hydrogen-to-helium ratios predicted from continuous accretion from the ISM in solar proportions at various rates, which are needed to reproduce the amount of hydrogen measured (or constrained) if the accretion starts at $T_{\text {eff }}=20,000 \mathrm{~K}$. The exact choice for the starting temperature is not critical since the cooling time scales increase rapidly with decreasing effective temperatures. We find that most DZ stars in Figure 29 with hydrogen abundance determinations are consistent with accretion rates in the range from $10^{-20}$ to $10^{-18} M_{\odot} \mathrm{yr}^{-1}$. This is about two to four orders

of magnitude smaller than the average metal accretion rate of $\sim 10^{-16} M_{\odot} \mathrm{yr}^{-1}$ used by Dupuis et al. (1993b). Our results confirm the conclusions reached by Dupuis et al. (1993b) and Wolff et al. (2002) that the hydrogen accretion rate must be at least two orders of magnitude lower that that of metals.

The conclusion that the hydrogen accretion rate must be significantly lower than that of metals is further demonstrated in Figure 30, where we show the $\mathrm{Ca} / \mathrm{H}$ abundance ratios as a function of effective temperature for our sample of DZ stars. The apparent correlation of $\mathrm{Ca} / \mathrm{H}$ with $T_{\text {eff }}$ is due in part to the fact that there are no stars with low calcium abundances at high effective temperatures, since the Ca II lines are not observable in this range of temperature (see Fig. 27). The dotted line indicates the solar $\mathrm{Ca} / \mathrm{H}$ abundance ratio. Because hydrogen can only accumulate with time in the mixed $\mathrm{H} / \mathrm{He}$ convection zone, the maximum $\mathrm{Ca} / \mathrm{H}$ abundance ratio can only become smaller with decreasing effective temperature. Thus, 
the observed $\mathrm{Ca} / \mathrm{H}$ abundance ratios that are close to the solar value in a few stars are only coincidental; this does not necessarily imply accretion in solar proportions, but indicates instead that the accretion of hydrogen was reduced relative to metals. The maximum value of $\mathrm{Ca} / \mathrm{H}$ that can be expected from accretion of material with solar composition is indicated by the solid line (from equation 6 of Dupuis et al. 1993b).

\subsection{Correlation with Effective Temperature}

More importantly, the results shown in Figure 29 reveal for the first time a correlation between the hydrogen abundances and the effective temperature. Such a correlation has never been observed before because the number of known DZ stars was simply too small. Moreover, as noted above, the absence of $\mathrm{H} \alpha$ in WD $0802+386$ near $T_{\text {eff }} \sim 11,000 \mathrm{~K}$ implies an unexpectedly low hydrogen abundance of $\log (\mathrm{H} / \mathrm{He})<-6.0$. We find seven additional DZ stars in the SDSS sample with $T_{\text {eff }}>9500 \mathrm{~K}$ and similar low hydrogen abundances. Figure 29 indicates that the accretion rate for these stars must be as low as $10^{-21} M_{\odot} \mathrm{yr}^{-1}$. An accretion rate slightly above $10^{-20} M_{\odot} \mathrm{yr}^{-1}$ would be sufficient to produce a detectable $\mathrm{H} \alpha$ absorption feature at these temperatures. The presence of such small amounts of hydrogen is very surprising, especially considering that several DZ stars in our sample do show $\mathrm{H} \alpha$ in the same temperature range. It seems very unlikely that white dwarfs experience such a diversity of average conditions while traveling large distances through the interstellar medium over their lifetime.

The inverse problem occurs at the other end of the diagram, where cool DZ stars with $\log (\mathrm{H} / \mathrm{He}) \sim-3$ can be accounted for with accretion rates around $10^{-19} M_{\odot} \mathrm{yr}^{-1}$ and above. However, DZ stars accreting hydrogen at such a high rate should be easily recognized at higher effective temperatures if the accretion rate has remained constant during the cooling of the star. Yet, our sample contains no hot $\left(T_{\text {eff }}>10,000 \mathrm{~K}\right)$ DZ star with high hydrogen abundances around $\log (\mathrm{H} / \mathrm{He}) \sim-4$ expected from the high accretion rate inferred from the cool DZ stars. Actually, the fact that the accretion rate required to explain the hydrogen abundances in Figure 29 increases from $\sim 10^{-20} M_{\odot} \mathrm{yr}^{-1}$ at $T_{\text {eff }}=11,000 \mathrm{~K}$ up to $\sim 10^{-19} M_{\odot} \mathrm{yr}^{-1}$ at $T_{\text {eff }}=7000 \mathrm{~K}$ could be interpreted as evidence that the physical mechanism that prevents hydrogen from being accreted onto the surface of these stars becomes less efficient with decreasing effective temperature. For instance, Wesemael \& Truran (1982) proposed a model where protons are prevented from accreting onto the surface of the white dwarf by a rotating magnetic field, while metals, most probably in the form of grains, are unaffected by this mechanism and thus reach the surface. Our results suggest that this so-called propeller mechanism may become less effective below $T_{\text {eff }} \sim 9000 \mathrm{~K}$. If 
this interpretation is correct, this would represent the first empirical evidence for a decrease in efficiency with temperature of the hydrogen screening mechanism at work in cool helium atmosphere white dwarfs. This mechanism is still operating quite efficiently, however, since the inferred accretion rates at low effective temperatures remain several orders of magnitude lower than those required to explain the presence of metals in DZ stars.

\subsection{Connection with Hotter DB Stars}

As mentioned above, $27 \%$ of the DZ stars in our sample contain hydrogen, a higher ratio than the $\sim 16 \%$ found in DBA stars (Shipman et al. 1987 gives 6/32, but after removal of the subdwarf PG $2224+034$, we get $5 / 31$, or $16 \%$ ). It is generally believed that this is the ratio among DB3 $\left(T_{\text {eff }} \sim 12,000-19,000 \mathrm{~K}\right)$ white dwarfs. However, these statistics for DB stars are based on the detection of $\mathrm{H} \beta$ and $\mathrm{H} \gamma$ only.

As part of a campaign undertaken by the Montreal group (see, e.g., Hunter et al. 2001), nearly 80 bright northern DB white dwarfs have been spectroscopically observed at medium and high resolutions. This sample also includes all known DB2 stars with spectra obtained at $\mathrm{H} \alpha$, a more sensitive line to study the ratio of DBA to DB stars. $\mathrm{H} \alpha$ is found in $7 / 18$ stars (or 38\%), more than twice the ratio obtained by Shipman et al. (1987) for the cooler DB3 stars. If this fraction for DB2 stars extends to the cooler DB3 stars, a systematic search for $\mathrm{H} \alpha$ in these objects is expected to perhaps double the number of DBA3 stars. Indeed, the atmospheres of DB stars become increasingly transparent with lower effective temperatures, which makes $\mathrm{H} \alpha$ easier to detect spectroscopically, even if the mass of the helium convection zone has significantly increased and further diluted the amount of hydrogen present in the photospheric regions.

We thus argue that the canonical $20 \%$ ratio of DBA to DB white dwarfs is certainly underestimated. Some authors have speculated that all DB stars must contain at least some traces of hydrogen, although explicit spectroscopic determinations are difficult (from observations of Ly $\alpha$, see Koester \& Weidemann 1989; Provencal et al. 2000), if not impossible, because the amounts of hydrogen expected are below the visibility limit. At the cooler end of the white dwarf sequence, Wolff et al. (2002) detected hydrogen in nearly all the DBZ and DZ stars in their sample. Several studies now favor the accretion of hydrogen from the ISM (as opposed to primordial origin) to explain the DBA phenomenon, since the amount of hydrogen found in cool DBA stars is incompatible with the scenario that involves the transformation of DA stars into DB stars near the red edge of the DB gap at $T_{\text {eff }} \sim 30,000 \mathrm{~K}$. However, a measure of the hydrogen accretion rate in DB stars is complicated by the fact that the mass of the helium convection zone varies rapidly with decreasing effective tem- 
perature, and also by the lack of accurate determinations of hydrogen abundances for large samples of DB and DBA stars.

Depending on the evolutionary models used, the total mass of hydrogen present in DBA stars ranges from about $10^{-14}$ to $10^{-11} M_{\odot}$. Such small amounts of hydrogen imply that cool helium-rich white dwarfs below $T_{\text {eff }} \sim 12,000 \mathrm{~K}$ must have completely forgotten their past hydrogen history as they become DC, DZ or DQ stars, since the increasing depth of the helium convection zone with decreasing effective temperature (see Fig. 10 of Tassoul et al. 1990) will dilute hydrogen to extremely small abundances, typically to $\log (\mathrm{H} / \mathrm{He})<-5$. Thus the evidence to date is that a large fraction - probably larger than $35 \%$ - of DB stars do accrete hydrogen at a rate we cannot yet estimate precisely, but which probably lies in the range from $10^{-22}$ to $10^{-19} M_{\odot} \mathrm{yr}^{-1}$, and that $\sim 27 \%$ of DZ stars have hydrogen abundances that can only be accounted for by invoking unusually low hydrogen accretion rates (this ratio for DZ stars will eventually go up when higher $\mathrm{S} / \mathrm{N}$ ratio spectroscopy around $\mathrm{H} \alpha$ becomes available for SDSS stars).

\subsection{Speculations on Spectral Evolution}

We discuss here an alternative, and very speculative scenario aimed at explaining various problems related to the abundance patterns observed in helium-rich white dwarfs. Koester (1976) argued that the accretion rate from the ISM should be lower than the fluid rate obtained from the Bondi-Hoyle equation since the physical conditions required for the hydrodynamic treatment are perhaps not met in a low interacting medium. If we assume that the accretion rate is indeed much lower than the fluid rate $\left(\dot{M}<10^{-21} M_{\odot} \mathrm{yr}^{-1}\right)$, we could naturally explain the low hydrogen abundances of $\log (\mathrm{H} / \mathrm{He})<-6$ determined for the hot DZ stars in our study. Such a low hydrogen accretion rate would also be compatible with the hydrogen abundance pattern observed in the hotter DB white dwarfs. The presence of metals in DZ stars would then have to be explained by other means than accretion from the ISM, such as cometary material or tidal disruption of planets or asteroids, models that are growing in popularity since the recent discoveries of infrared excesses in DAZ white dwarfs (see, e.g., Kilic et al. 2006, and references therein).

In Figure 31, we show the results already displayed in Figure 29 in a different way by plotting as a function of effective temperature the total mass of hydrogen present in each star, obtained by combining the mass of the helium convection zone at a given effective temperature with our determinations of the $\mathrm{H} / \mathrm{He}$ abundance ratios. Also reproduced in Figure 31 are the hydrogen layer masses predicted for continuous accretion from the ISM in solar proportions at various rates needed to account for the amount of hydrogen measured 
(or constrained) if the accretion starts at $T_{\text {eff }}=20,000 \mathrm{~K}$.

We speculate here that the high hydrogen abundances, $\log (\mathrm{H} / \mathrm{He})=-5$ to -3 , observed in a fraction of our DZA stars could be explained in terms of convectively mixed DA stars at low effective temperatures. This process is believed to occur when the bottom of the thin hydrogen convection zone in a hydrogen-atmosphere white dwarf penetrates the deeper and more massive underlying helium layer. Figure 32 illustrates the extent of the hydrogen convection zone as a function of effective temperature in a $0.6 M_{\odot}$ DA white dwarf. A DA star with a very thin hydrogen layer of $q(\mathrm{H}) \equiv \Delta M / M_{\star}=10^{-11}$ would thus mix at an effective temperature near $10,000 \mathrm{~K}$, while a DA star with a much thicker hydrogen layer of $q(\mathrm{H})=10^{-8}$ would mix at a lower temperature of $\sim 6500 \mathrm{~K}$. The mixing process, although not yet well understood, would presumably turn a hydrogen-atmosphere white dwarf into a helium-dominated atmosphere with only small traces of hydrogen, since the mass of the helium convection zone is much larger than the hydrogen layer mass. Although there are no detailed quantitative calculations available in the literature, the expected $\mathrm{H} / \mathrm{He}$ abundance ratios as a function of effective temperature would follow, at least qualitatively, the abundance pattern observed in Figures 29 and 31 .

If the convective mixing scenario proposed here to explain the hydrogen abundances observed in cool DZ stars survives closer examination, it could also naturally explain the absence of a correlation between the position and motion of DZ stars with the distribution of local interstellar matter, as discussed by Aannestad et al. (1993). The mixing scenario could also provide an explanation for another fact that has not received much attention in the literature: the lack of DQ white dwarfs with hydrogen abundances comparable to those observed in DZ stars. If we combine the 40 new DQ stars analyzed by Koester \& Knist (2006) with the 56 analyzed by Dufour et al. (2005) (and other DQ stars from the literature), we have a sample of over a hundred DQ stars, none of which show $\mathrm{H} \alpha$. We note that the increased opacity provided by carbon affects the detection limit of hydrogen features compared to DZA stars, especially for DQ stars with high carbon abundances $(\log (\mathrm{C} / \mathrm{He})>-4)$, but according to our models, $\mathrm{H} \alpha$ should still be easily detected in DQ stars with $\log (\mathrm{H} / \mathrm{He}) \sim-4$ to -3 at $T_{\text {eff }}=7000-9000 \mathrm{~K}$. In fact, only one DQ star is known to contain hydrogen, G99-37, and it is only indirectly detected from the presence of a $\mathrm{CH}$ molecular band. If accretion from the interstellar medium is responsible for the presence of hydrogen in DZ white dwarfs, we would then expect at least a few DQ stars to show $\mathrm{H} \alpha$. We propose instead that DQ white dwarfs do not show large amounts of hydrogen in their atmospheres simply because they are the direct descendants of the DB and DBA stars, while DZ and DZA white dwarfs originate respectively from two separate populations of white dwarfs, namely DB stars that have not accreted a detectable quantity of hydrogen and convectively mixed DA stars. This scenario also predicts that the proportion of DZA stars should increase with decreasing effective 
temperature. However, it is premature to test this idea with our sample since too many stars may have small traces of hydrogen that will become detectable only through higher $\mathrm{S} / \mathrm{N}$ ratio spectroscopy.

\section{SUMMARY AND CONCLUSIONS}

We presented a detailed photometric and spectroscopic analysis of 159 DZ white dwarfs drawn from two samples (12 stars from BRL97 and BLR01, and 147 from the SDSS). This is more than a ninefold increase in the number of DZ stars ever analyzed and represents the largest set analyzed in a homogeneous fashion.

Our reanalysis of the DZ stars from the BRL97/BLR01 sample reveals that the effective temperatures and stellar masses derived with models including hydrogen and metals are significantly different from the values obtained from pure helium models. For instance, our mean mass for the $11 \mathrm{DZ}$ stars with trigonometric parallax measurements, $\langle M\rangle=0.63 M_{\odot}$, is significantly lower than the average mass obtained by BRL97/BLR01, $\langle M\rangle=0.78 M_{\odot}$, and closer to the mean mass of other types of white dwarfs. The atmospheric parameter determinations for the coolest DZ stars in our sample are more uncertain due to possible pressure effects in white dwarf atmospheres below $T_{\text {eff }} \sim 6000 \mathrm{~K}$. As such, the coolest DZ stars represent a useful probe of non-ideal effects at high gas densities.

Hydrogen is present in 43 of the 159 DZ stars (or 27\%) in our complete sample, a fraction that will most certainly rise when higher signal-to-noise follow-up spectroscopy near $\mathrm{H} \alpha$ becomes available for SDSS objects. Our analysis also revealed for the first time a correlation between the hydrogen abundance and the effective temperature of DZ stars. The amount of hydrogen measured in the photospheric regions of DZ stars can be explained from accretion from the ISM only if the accretion rate is at least two (and possibly up to five) orders of magnitude lower than the corresponding rate for metals. Also, the hydrogen

accretion rate inferred from the results of our analysis suggests an increase in the rate of about one order of magnitude when the effective temperature decreases, providing perhaps the first empirical evidence of a lower temperature boundary for the hydrogen screening mechanism. We finally speculated about an alternative scenario where the hydrogen pattern in DZA white dwarfs could be explained as the result of the convective mixing of the thin hydrogen layer in DA stars with the more massive underlying helium convective zone.

We wish to thank D. Koester for providing us with his theoretical Ly $\alpha$ line profile calculations, A. Gianninas for a careful reading of our manuscript, and an anonymous referee for many constructive comments. We would also like to thank the director and staff of 
Steward Observatory for the use of their facilities. This work was supported in part by the NSERC Canada. P. Bergeron is a Cottrell Scholar of Research Corporation.

Funding for the SDSS and SDSS-II has been provided by the Alfred P. Sloan Foundation, the Participating Institutions, the National Science Foundation, the U.S. Department of Energy, the National Aeronautics and Space Administration, the Japanese Monbukagakusho, the Max Planck Society, and the Higher Education Funding Council for England.

The SDSS is managed by the Astrophysical Research Consortium for the Participating Institutions. The Participating Institutions are the American Museum of Natural History, Astrophysical Institute Potsdam, University of Basel, Cambridge University, Case Western Reserve University, University of Chicago, Drexel University, Fermilab, the Institute for Advanced Study, the Japan Participation Group, Johns Hopkins University, the Joint Institute for Nuclear Astrophysics, the Kavli Institute for Particle Astrophysics and Cosmology, the Korean Scientist Group, the Chinese Academy of Sciences (LAMOST), Los Alamos National Laboratory, the Max-Planck-Institute for Astronomy (MPIA), the Max-Planck-Institute for Astrophysics (MPA), New Mexico State University, Ohio State University, University of Pittsburgh, University of Portsmouth, Princeton University, the United States Naval Observatory, and the University of Washington.

\section{REFERENCES}

Aannestad, P. A., Kenyon, S. J., Hammond, G. L., \& Sion, E. M. 1993, AJ, 105, 1033

Abazajian, K., et al. 2003, AJ, 126, 2081

Adelman-McCarthy, J. K., et al. 2006, ApJS, 162, 38

Beauchamp, A. 1995, Ph.D. Thesis, Université de Montréal

Becklin, E. E., Farihi, J., Jura, M., Song, I., Weinberger, A. J., \& Zuckerman, B. 2005, ApJ, 632, L119

Bergeron, P., Leggett, S. K., \& Ruiz, M. T. 2001, ApJS, 133, 413 (BLR01)

Bergeron, P., Ruiz, M. T., \& Leggett, S. K. 1997, ApJS, 108, 339 (BRL97)

Bergeron, P., Saumon, D., \& Wesemael, F. 1995, ApJ, 443, 764

Cottrell, P. L., \& Greenstein, J. L. 1980a, ApJ, 238, 941

Cottrell, P. L., \& Greenstein, J. L. 1980b, ApJ, 242, 195 
Dufour, P., Bergeron, P., \& Fontaine, G. 2005, ApJ, 627, 404

Dufour, P., Bergeron, P., Schmidt, G. D., Liebert, J., Harris, H. C., Knapp, G. R., Anderson, S. F., \& Schneider, D. P. 2006, ApJ, 651, 1112

Dupuis, J., Fontaine, G., Pelletier, C., \& Wesemael, F. 1992, ApJS, 82, 505

Dupuis, J., Fontaine, G., Pelletier, C., \& Wesemael, F. 1993a, ApJS, 84, 73

Dupuis, J., Fontaine, G., \& Wesemael, F. 1993b, ApJS, 87, 345

Eisenstein, D. J., et al. 2006, ApJS, 167, 40

Fontaine, G., Brassard, P., \& Bergeron, P. 2001, PASP, 113, 409

Fukugita, M., Ichikawa, T., Gunn, J. E., Doi, M., Shimasaku, K., \& Schneider, D. P. 1996, AJ, 111, 1748

Grenfell, T. C. 1974, A\&A, 31, 303

Gunn, J. E., et al. 1998, AJ, 116, 3040

Gunn, J. E., et al. 2006, AJ, 131, 2332

Harris, H. C., et al. 2003, AJ, 126, 1023

Hogg, D. W., Finkbeiner, D. P., Schlegel, D.J., \& Gunn, J. E., 2001, AJ, 122, 2129

Hummer, D. G., \& Mihalas, D. 1988, ApJ, 331, 794

Hunter, C., Wesemael, F., Saffer, R. A., Bergeron, P., \& Beauchamp, A. 2001, in ASP Conf. Ser. Vol. 226, 12th European Workshop on White Dwarf Stars, eds. J. L. Provencal, H. L. Shipman, J. MacDonald, \& S. Goodchild, 153

Ivezić, Z., et al. 2004, Astron. Nachr., 325, 583

Kapranidis, S., \& Liebert, J. 1986, ApJ, 305, 863

Kilic, M., von Hippel, T., Leggett, S. K., \& Winget, D. E. 2005, ApJ, 632, L115

Kilic, M., et al. 2006a, AJ, 131, 582

Kilic, M., von Hippel, T., Leggett, S. K., \& Winget, D. E. 2006b, ApJ, 646, 474

Kleinman, S. J., et al. 2004, ApJ, 607, 426 
Koester, D. 1976, A\&A, 52, 415

Koester, D., \& Knist, S. 2006, A\&A, 454, 951

Koester, D., Wegner, G., \& Kilkenny, D. 1990, ApJ, 350, 329

Koester, D., \& Weidemann, V. 1989, A\&A, 219, 276

Koester, D., \& Wilken, D. 2006, A\&A, 453, 1051

Koester, D., \& Wolff, B. 2000, A\&A, 357, 587

Liebert, J. 1977, A\&A, 60, 101

Liebert, J., Wehrse, R., \& Green, R. F. 1987, A\&A, 175, 173

Paquette, C., Pelletier, C., Fontaine, G., \& Michaud, G. 1986, ApJS, 61, 197

Pier, J. R., Munn, J. A., Hindsley, R. B., Hennessy, G. S., Kent, S. M., Lupton, R. H., \& Ivezić, Ž. 2003, AJ, 125, 1559

Press, W. H., Teukolsky, S. A., Vetterling, W. T., \& Flannery, B. P. 1992, Numerical Recipes in FORTRAN, 2nd edition (Cambridge: Cambridge University Press), 644

Provencal, J. L., Shipman, H. L., Koester, D., Wesemael, F., \& Bergeron, P. 2002, ApJ, 568, 324

Provencal, J. L., Shipman, H. L., Thejll, P., \& Vennes, S. 2000, ApJ, 542, 1041

Reach, W. T., Kuchner, M. J., von Hippel, T., Burrows, A., Mullally, F., Kilic, M., \& Winget, D. E. 2005, ApJ, 635, L161

Richards, G. T., et al. 2002, AJ, 123, 2945

Saumon, D., Chabrier, G., \& Van Horn, H. M. 1995, ApJS, 99, 713

Shipman, H. L., Liebert, J., \& Green, R. F. 1987, ApJ, 315, 239

Sion, E. M., Hammond, G. L., Wagner, R. M., Starrfield, S. G., \& Liebert, J. 1990, ApJ, 362,691

Sion, E. M., Kenyon, S. J., \& Aannestad, P. A. 1990, ApJS, 72, 707

Smith, J. A., et al. 2002, AJ, 123, 2121

Stoughton, C., et al. 2002, AJ, 123, 485 
Tassoul, M., Fontaine, G., \& Winget, D.E. 1990, ApJS, 72, 335

Tucker, D. L., et al. 2006, Astronomische Nachrichten, 327, 821

Wegner, G. 1972, ApJ, 172, 451

Wehrse, R., \& Liebert, J. 1980, A\&A, 86, 139

Weidemann, V. 1960, ApJ, 131, 638

Weidemann, V., \& Koester, D. 1989, A\&A, 210, 311

Wesemael, F., Greenstein, J. L., Liebert, J., Lamontagne, R., Fontaine, G., Bergeron, P., \& Glaspey, J. W. 1993, PASP, 105, 761

Wesemael, F., \& Truran, J. W. 1982, ApJ, 260, 807

Wolff, B., Koester, D., \& Liebert, J. 2002, A\&A, 385, 995

York, D. G., et al. 2000, AJ, 120, 1579

Zeidler-K.T., E.-M., Weidemann, V., \& Koester, D. 1986, A\&A, 155, 356 
Table 1. Atmospheric Parameters of DZ Stars from the BRL97 and BLR01 Samples

\begin{tabular}{clccccc}
\hline \hline WD & \multirow{2}{*}{ Name } & $T_{\text {eff }}(\mathrm{K})$ & $\log g$ & $M / M_{\odot}$ & $\log (\mathrm{Ca} / \mathrm{He})$ & $\log (\mathrm{H} / \mathrm{He})$ \\
\hline $0046+051$ & vMa 2 & $6220(240)$ & $8.19(0.04)$ & $0.69(0.02)$ & $-10.00(0.05)$ & $-3.19(0.18)^{\mathrm{a}}$ \\
$0552-041$ & LP 658-2 & $4270(70)$ & $7.80(0.02)$ & $0.45(0.01)$ & $-10.92(0.04)$ & $<-5.0$ \\
& & $4350(60)$ & $7.87(0.02)$ & $0.49(0.01)$ & $-10.99(0.03)$ & no H \\
$0738-172$ & L745-46A & $7590(220)$ & $8.07(0.03)$ & $0.62(0.02)$ & $-10.91(0.03)$ & $-3.41(0.03)$ \\
$0802+386$ & LP 257-28 & $10980(490)$ & $8.31(0.19)$ & $0.78(0.12)$ & $-9.76(0.09)$ & $<-6.0$ \\
$1313-198$ & LHS 2710 & $4520(160)$ & $7.86(0.08)$ & $0.48(0.04)$ & $-11.18(0.03)$ & $<-5.0$ \\
& & $4570(90)$ & $7.89(0.08)$ & $0.50(0.04)$ & $-11.23(0.04)$ & no H \\
$1328+307$ & G165-7 & $6440(210)$ & $7.99(0.29)$ & $0.57(0.17)$ & $-8.10(0.15)$ & $-3.0(0.20)$ \\
$1338-311$ & ESO 445-271 & $8210(460)$ & 8.00 & 0.58 & $-10.03(0.02)$ & $<-4.0$ \\
& & $8560(600)$ & 8.00 & 0.58 & $-10.43(0.02)$ & no H \\
$1626+368$ & Ross 640 & $8440(320)$ & $8.02(0.05)$ & $0.59(0.03)$ & $-8.83(0.04)$ & $-3.63(0.05)$ \\
$1705+030$ & G139-13 & $6580(200)$ & $8.20(0.15)$ & $0.70(0.09)$ & $-10.05(0.05)$ & $-3.55(0.14)^{\mathrm{a}}$ \\
$2251-070$ & LP 701-29 & $4000(200)$ & $8.01(0.06)$ & $0.58(0.04)$ & $-10.45(0.02)$ & $<-6.0$ \\
$2312-024$ & LHS 3917 & $6220(190)$ & $8.19(0.26)$ & $0.69(0.16)$ & $-10.55(0.05)$ & $-4.90(0.30)^{\mathrm{a}}$ \\
$2345-447$ & ESO 292-43 & $4620(110)$ & $8.35(0.09)$ & $0.80(0.06)$ & $-11.35(0.04)$ & $<-5.0$ \\
& & $4650(70)$ & $8.36(0.08)$ & $0.81(0.06)$ & $-11.43(0.04)$ & no H \\
\hline
\end{tabular}

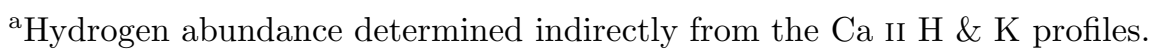


Table 2. Atmospheric Parameters of DZ Stars from the SDSS

\begin{tabular}{|c|c|c|c|c|c|c|c|}
\hline Name & Plate & MJD & Fiber & $T_{\text {eff }}(\mathrm{K})$ & $\log (\mathrm{Ca} / \mathrm{He})$ & $D(\mathrm{pc})$ & $\log (\mathrm{H} / \mathrm{He})$ \\
\hline SDSS J000557.20+001833.3 & 388 & 51793 & 394 & $7970(190)$ & $-9.62(0.07)$ & 136 & $<-4.0$ \\
\hline SDSS J001849.43+001204.7 & 1491 & 52996 & 27 & $9600(170)$ & $-9.83(0.07)$ & 157 & $<-5.0$ \\
\hline SDSS J003601.38-111214.0 & 655 & 52162 & 300 & $7280(70)$ & $-9.26(0.04)$ & 55 & $<-4.0$ \\
\hline SDSS J004123.58+151109.0 & 419 & 51868 & 395 & $7770(170)$ & $-10.72(0.16)$ & 177 & $<-4.0$ \\
\hline SDSS J004646.16+002430.9 & 691 & 52199 & 503 & $8770(220)$ & $-8.57(0.09)$ & 225 & $<-4.0$ \\
\hline SDSS J005906.77+001725.2 & 395 & 51783 & 508 & $10400(420)$ & $-9.80(0.19)$ & 248 & $<-5.0$ \\
\hline SDSS J010629.85-010344.2 & 396 & 51816 & 55 & $10240(350)$ & $-9.21(0.09)$ & 192 & $-4.30(0.13)$ \\
\hline SDSS J011338.35+000632.7 & 694 & 52209 & 24 & $7840(160)$ & $-9.79(0.09)$ & 179 & $<-4.0$ \\
\hline SDSS J011358.98-095913.3 & 660 & 52177 & 277 & $10610(290)$ & $-8.87(0.05)$ & 130 & $-5.06(0.06)$ \\
\hline SDSS J012339.77+132433.4 & 424 & 51893 & 132 & $7300(270)$ & $-9.44(0.22)$ & 223 & $<-3.0$ \\
\hline SDSS J013831.12+003101.6 & 698 & 52203 & 500 & $9060(270)$ & $-10.34(0.11)$ & 225 & $<-5.0$ \\
\hline SDSS J020001.99+004018.4 & 701 & 52179 & 430 & $9860(170)$ & $-9.90(0.09)$ & 173 & $<-5.0$ \\
\hline SDSS J020132.24-003932.0 & 404 & 51812 & 303 & $9650(210)$ & $-8.33(0.05)$ & 110 & $<-5.0$ \\
\hline SDSS J021836.70-091945.0 & 668 & 52162 & 138 & $9560(300)$ & $-10.63(0.07)$ & 97 & $<-5.0$ \\
\hline SDSS J022851.97-000938.8 & 406 & 51869 & 31 & $8310(310)$ & $-8.08(0.22)$ & 279 & $<-3.0$ \\
\hline SDSS J030800.40-065659.9 & 459 & 51924 & 345 & $8520(400)$ & $-8.81(0.23)$ & 285 & $<-3.0$ \\
\hline SDSS J031448.24-082755.2 & 459 & 51924 & 46 & $10290(610)$ & $-9.51(0.31)$ & 358 & $<-5.0$ \\
\hline SDSS J041145.90-054848.5 & 465 & 51910 & 52 & $9320(580)$ & $-8.95(0.32)$ & 359 & $<-3.0$ \\
\hline SDSS J073835.97+384438.2 & 431 & 51877 & 601 & $9530(360)$ & $-8.36(0.13)$ & 271 & $-4.42(0.35)$ \\
\hline SDSS J074743.56+400110.1 & 432 & 51884 & 36 & $8520(200)$ & $-10.87(0.17)$ & 161 & $<-4.0$ \\
\hline SDSS J074751.39+373217.0 & 433 & 51873 & 13 & $9600(390)$ & $-10.00(0.14)$ & 269 & $<-5.0$ \\
\hline SDSS J074821.86+350648.7 & 542 & 51993 & 515 & $10130(430)$ & $-10.43(0.18)$ & 356 & $<-5.0$ \\
\hline SDSS J074942.87+312424.6 & 890 & 52583 & 268 & $6910(80)$ & $-9.86(0.13)$ & 102 & $-3.14(0.23)^{\mathrm{a}}$ \\
\hline SDSS J074958.26+434306.0 & 434 & 51885 & 401 & $8950(470)$ & $-9.62(0.18)$ & 350 & $<-5.0$ \\
\hline SDSS J075846.90+322523.3 & 890 & 52583 & 588 & $10090(460)$ & $-10.24(0.20)$ & 336 & $<-5.0$ \\
\hline SDSS J080211.42+301256.7 & 860 & 52319 & 388 & $9400(470)$ & $-9.17(0.19)$ & 385 & $<-4.0$ \\
\hline SDSS J080331.46+450257.8 & 439 & 51877 & 351 & $9290(320)$ & $-9.53(0.23)$ & 276 & $-3.85(0.28)$ \\
\hline SDSS J080537.64+383212.4 & 758 & 52253 & 383 & $10660(40)$ & $-10.03(0.03)$ & 49 & $<-6.0$ \\
\hline SDSS J080602.91+374720.6 & 758 & 52253 & 122 & $9430(280)$ & $-8.38(0.14)$ & 244 & $<-5.0$ \\
\hline SDSS J080615.25+364018.1 & 758 & 52253 & 80 & $10130(570)$ & $-9.23(0.24)$ & 389 & $<-5.0$ \\
\hline
\end{tabular}


Table 2-Continued

\begin{tabular}{|c|c|c|c|c|c|c|c|}
\hline Name & Plate & MJD & Fiber & $T_{\text {eff }}(\mathrm{K})$ & $\log (\mathrm{Ca} / \mathrm{He})$ & $D(\mathrm{pc})$ & $\log (\mathrm{H} / \mathrm{He})$ \\
\hline SDSS J082720.59+330437.9 & 932 & 52620 & 372 & $7870(210)$ & $-8.97(0.12)$ & 221 & $<-5.0$ \\
\hline SDSS J082927.85+075911.4 & 1758 & 53084 & 154 & $9670(180)$ & $-8.68(0.07)$ & 176 & $<-5.0$ \\
\hline SDSS J083434.68+464130.6 & 549 & 51981 & 560 & $6610(100)$ & $-9.85(0.06)$ & 110 & $<-5.0$ \\
\hline SDSS J083556.31+090619.4 & 1759 & 53081 & 338 & $8390(150)$ & $-9.35(0.09)$ & 165 & $<-4.0$ \\
\hline SDSS J084200.24+362540.0 & 864 & 52320 & 101 & $10320(240)$ & $-9.08(0.08)$ & 218 & $-4.78(0.13)$ \\
\hline SDSS J084502.70+411547.6 & 829 & 52296 & 367 & $8300(220)$ & $-8.55(0.11)$ & 218 & $<-4.0$ \\
\hline SDSS J084525.00+535208.8 & 446 & 51899 & 176 & $7180(160)$ & $-10.71(0.13)$ & 144 & $<-3.0$ \\
\hline SDSS J084709.11+450734.8 & 763 & 52235 & 146 & $8520(350)$ & $-9.47(0.18)$ & 267 & $<-4.0$ \\
\hline SDSS J084828.00+521422.5 & 447 & 51877 & 499 & $8330(190)$ & $-10.83(0.05)$ & 110 & $<-5.0$ \\
\hline SDSS J084849.42+354857.8 & 934 & 52672 & 553 & $8090(80)$ & $-10.46(0.04)$ & 105 & $<-4.0$ \\
\hline SDSS J084857.88+002834.9 & 467 & 51901 & 150 & $11950(320)$ & $-8.25(0.13)$ & 221 & $<-6.0$ \\
\hline SDSS J084906.69+071030.0 & 1298 & 52964 & 29 & $8320(260)$ & $-8.37(0.14)$ & 237 & $-3.36(0.25)$ \\
\hline SDSS J084911.86+403649.7 & 830 & 52293 & 253 & $9230(140)$ & $-10.35(0.07)$ & 134 & $<-5.0$ \\
\hline SDSS J085141.72+053852.1 & 1189 & 52668 & 139 & $7480(240)$ & $-9.03(0.14)$ & 212 & $<-4.0$ \\
\hline SDSS J090517.71+013307.6 & 471 & 51924 & 221 & $8180(190)$ & $-10.21(0.11)$ & 193 & $<-4.0$ \\
\hline SDSS J090556.22+523533.1 & 553 & 51999 & 380 & $7510(180)$ & $-9.15(0.09)$ & 151 & $<-4.0$ \\
\hline SDSS J091643.07+010531.5 & 472 & 51955 & 577 & $7500(340)$ & $-9.77(0.24)$ & 240 & $<-3.0$ \\
\hline SDSS J092801.78+612434.1 & 485 & 51909 & 527 & $8570(340)$ & $-8.71(0.21)$ & 288 & $<-4.0$ \\
\hline SDSS J093210.54+485601.7 & 901 & 52641 & 259 & $8210(250)$ & $-8.71(0.19)$ & 262 & $<-4.0$ \\
\hline SDSS J093423.17+082225.3 & 1304 & 52993 & 222 & $9130(110)$ & $-9.82(0.03)$ & 117 & $<-5.0$ \\
\hline SDSS J093545.45+003750.9 & 476 & 52314 & 417 & $9030(470)$ & $-8.67(0.22)$ & 369 & $<-4.0$ \\
\hline SDSS J093704.99+364647.2 & 1275 & 52996 & 114 & $11190(430)$ & $-9.94(0.26)$ & 263 & $-4.89(0.18)$ \\
\hline SDSS J093942.30+555048.7 & 556 & 51991 & 167 & $8680(80)$ & $-8.51(0.02)$ & 65 & $-4.20(0.05)$ \\
\hline SDSS J094148.75+502214.5 & 901 & 52641 & 590 & $10940(420)$ & $-9.12(0.17)$ & 294 & $<-5.0$ \\
\hline SDSS J094206.21+575556.0 & 452 & 51911 & 32 & $11430(400)$ & $-8.54(0.23)$ & 312 & $<-6.0$ \\
\hline SDSS J094210.50+074354.7 & 1234 & 52724 & 220 & $9140(190)$ & $-9.32(0.09)$ & 190 & $<-5.0$ \\
\hline SDSS J094415.33+393943.0 & 1215 & 52725 & 576 & $11190(560)$ & $-8.97(0.14)$ & 314 & $<-6.0$ \\
\hline SDSS J094451.59+440856.7 & 941 & 52709 & 326 & $10460(470)$ & $-9.35(0.22)$ & 288 & $<-5.0$ \\
\hline SDSS J094530.20+084624.8 & 1234 & 52724 & 514 & $10550(220)$ & $-10.03(0.15)$ & 227 & $<-6.0$ \\
\hline SDSS J094743.09+423841.3 & 941 & 52709 & 131 & $9680(320)$ & $-8.50(0.14)$ & 270 & $<-4.0$ \\
\hline
\end{tabular}


Table 2-Continued

\begin{tabular}{|c|c|c|c|c|c|c|c|}
\hline Name & Plate & MJD & Fiber & $T_{\text {eff }}(\mathrm{K})$ & $\log (\mathrm{Ca} / \mathrm{He})$ & $D(\mathrm{pc})$ & $\log (\mathrm{H} / \mathrm{He})$ \\
\hline SDSS J095119.85+403322.4 & 1216 & 52709 & 141 & $8370(60)$ & $-10.27(0.04)$ & 84 & $-4.16(0.13)$ \\
\hline SDSS J095435.86+563518.2 & 557 & 52253 & 105 & $8940(260)$ & $-9.79(0.13)$ & 219 & $<-5.0$ \\
\hline SDSS J095645.15+591240.6 & 453 & 51915 & 621 & $8230(80)$ & $-7.54(0.06)$ & 128 & $-3.20(0.09)$ \\
\hline SDSS J100237.36+031325.5 & 573 & 52325 & 291 & $9800(200)$ & $-9.19(0.12)$ & 212 & $<-5.0$ \\
\hline SDSS J100406.64+423151.3 & 1217 & 52672 & 577 & $8270(340)$ & $-9.98(0.14)$ & 242 & $<-4.0$ \\
\hline SDSS J100421.25+045117.2 & 573 & 52325 & 363 & $7910(180)$ & $-9.21(0.13)$ & 193 & $<-4.0$ \\
\hline SDSS J100823.75+024840.0 & 502 & 51957 & 325 & $7940(220)$ & $-11.26(0.12)$ & 120 & $<-4.0$ \\
\hline SDSS J101007.84+394852.2 & 1356 & 53033 & 640 & $8470(90)$ & $-8.66(0.05)$ & 128 & $-3.94(0.16)$ \\
\hline SDSS J101558.21+414131.7 & 1357 & 53034 & 523 & $9390(200)$ & $-8.90(0.10)$ & 206 & $-4.66(0.25)$ \\
\hline SDSS J101805.17+034435.6 & 574 & 52355 & 104 & $8690(370)$ & $-8.62(0.17)$ & 275 & $<-4.0$ \\
\hline SDSS J103126.19+120340.4 & 1599 & 53089 & 455 & $8350(70)$ & $-8.62(0.04)$ & 102 & $<-4.0$ \\
\hline SDSS J103300.11+624747.8 & 772 & 52375 & 542 & $7590(120)$ & $-9.92(0.45)$ & 137 & $-3.71(0.89)^{\mathrm{a}}$ \\
\hline SDSS J103651.09+483754.0 & 875 & 52354 & 122 & $11460(330)$ & $-9.02(0.20)$ & 292 & $-5.21(0.26)$ \\
\hline SDSS J103809.19-003622.5 & 274 & 51913 & 265 & $6770(30)$ & $-9.44(0.06)$ & 49 & $<-5.0$ \\
\hline SDSS J103941.86+461224.3 & 962 & 52620 & 132 & $6770(120)$ & $-10.05(0.17)$ & 109 & $-5.62(0.55)^{\mathrm{a}}$ \\
\hline SDSS J104511.21+625442.2 & 773 & 52376 & 554 & $9020(470)$ & $-9.77(0.32)$ & 340 & $<-4.0$ \\
\hline SDSS J104911.53+515423.5 & 1010 & 52649 & 84 & $6680(40)$ & $-9.43(0.05)$ & 72 & $-4.41(0.24)^{\mathrm{a}}$ \\
\hline SDSS J104915.06-000706.2 & 275 & 51910 & 111 & $8680(160)$ & $-8.53(0.05)$ & 171 & $-3.91(0.14)$ \\
\hline SDSS J105221.56+065915.4 & 1001 & 52670 & 557 & $11000(440)$ & $-9.60(0.32)$ & 317 & $<-5.0$ \\
\hline SDSS J105601.50+012825.0 & 507 & 52353 & 14 & $10200(240)$ & $-9.24(0.06)$ & 122 & $-4.76(0.08)$ \\
\hline SDSS J105616.90-000449.4 & 277 & 51908 & 311 & $10950(460)$ & $-9.17(0.25)$ & 355 & $-4.63(0.32)$ \\
\hline SDSS J105641.71+571448.9 & 949 & 52427 & 99 & $7420(70)$ & $-10.70(0.08)$ & 99 & $<-4.0$ \\
\hline SDSS J105853.70+604136.8 & 774 & 52286 & 262 & $8870(460)$ & $-8.96(0.32)$ & 316 & $<-4.0$ \\
\hline SDSS J110438.38+071129.8 & 1003 & 52641 & 172 & $10910(160)$ & $-10.45(0.15)$ & 169 & $<-6.0$ \\
\hline SDSS J111215.05+070052.3 & 1004 & 52723 & 111 & $6890(120)$ & $-9.02(0.08)$ & 142 & $<-3.0$ \\
\hline SDSS J112258.34+504146.7 & 879 & 52365 & 289 & $8740(200)$ & $-7.93(0.12)$ & 217 & $-4.40(0.34)$ \\
\hline SDSS J112617.16+524155.1 & 879 & 52365 & 407 & $9460(150)$ & $-9.50(0.06)$ & 159 & $-4.86(0.16)$ \\
\hline SDSS J112956.98-015229.5 & 326 & 52375 & 590 & $8480(190)$ & $-9.04(0.10)$ & 192 & $-3.33(0.17)$ \\
\hline SDSS J113001.61+103614.8 & 1223 & 52781 & 570 & $8620(170)$ & $-8.59(0.08)$ & 168 & $<-4.0$ \\
\hline SDSS J113711.28+034324.7 & 838 & 52378 & 292 & $7120(60)$ & $-10.91(0.08)$ & 73 & $<-3.0$ \\
\hline
\end{tabular}


Table 2-Continued

\begin{tabular}{|c|c|c|c|c|c|c|c|}
\hline Name & Plate & MJD & Fiber & $T_{\text {eff }}(\mathrm{K})$ & $\log (\mathrm{Ca} / \mathrm{He})$ & $D(\mathrm{pc})$ & $\log (\mathrm{H} / \mathrm{He})$ \\
\hline SDSS J114054.87+532827.4 & 1015 & 52734 & 95 & $9860(250)$ & $-8.54(0.12)$ & 232 & $-4.51(0.23)$ \\
\hline SDSS J114712.18+492801.0 & 967 & 52636 & 28 & $7810(80)$ & $-9.70(0.05)$ & 111 & $<-4.0$ \\
\hline SDSS J115547.42+432751.4 & 1447 & 53120 & 538 & $8390(120)$ & $-10.46(0.07)$ & 125 & $-4.00(0.00)^{\mathrm{a}}$ \\
\hline SDSS J121218.69+540938.7 & 1019 & 52707 & 293 & $8710(140)$ & $-9.41(0.08)$ & 142 & $<-5.0$ \\
\hline SDSS J121837.12+002304.0 & 288 & 52000 & 423 & $6090(100)$ & $-9.92(0.10)$ & 122 & $<-4.0$ \\
\hline SDSS J122204.48+634354.5 & 780 & 52370 & 367 & $8790(340)$ & $-10.11(0.17)$ & 247 & $<-4.0$ \\
\hline SDSS J122733.45+633029.5 & 780 & 52370 & 443 & $7270(60)$ & $-8.71(0.04)$ & 95 & $<-4.0$ \\
\hline SDSS J122929.04+425414.4 & 1452 & 53112 & 541 & $11090(690)$ & $-9.48(0.33)$ & 429 & $<-5.0$ \\
\hline SDSS J122953.17+512925.2 & 884 & 52374 & 94 & $9230(250)$ & $-9.60(0.16)$ & 218 & $<-4.0$ \\
\hline SDSS J123455.96-033047.1 & 335 & 52000 & 247 & $8860(180)$ & $-10.83(0.06)$ & 114 & $<-5.0$ \\
\hline SDSS J124006.36-003700.9 & 290 & 51941 & 24 & $8370(170)$ & $-9.23(0.09)$ & 118 & $<-5.0$ \\
\hline SDSS J125413.79-023608.5 & 337 & 51997 & 153 & $6960(120)$ & $-10.45(0.19)$ & 89 & $-3.34(0.50)^{\mathrm{a}}$ \\
\hline SDSS J130746.34+030742.0 & 524 & 52027 & 589 & $8170(180)$ & $-9.64(0.06)$ & 130 & $-3.55(0.10)$ \\
\hline SDSS J130905.26+491359.7 & 1281 & 52753 & 439 & $8620(60)$ & $-10.16(0.03)$ & 83 & $<-5.0$ \\
\hline SDSS J131336.96+573800.5 & 1319 & 52791 & 409 & $8900(50)$ & $-9.39(0.02)$ & 68 & $-4.70(0.09)$ \\
\hline SDSS J132506.96+652132.2 & 603 & 52056 & 401 & $11650(370)$ & $-8.97(0.19)$ & 262 & $-4.41(0.21)$ \\
\hline SDSS J133824.97-013017.1 & 912 & 52427 & 457 & $10730(120)$ & $-9.44(0.06)$ & 147 & $<-6.0$ \\
\hline SDSS J134144.10-011238.4 & 299 & 51671 & 137 & $8090(220)$ & $-8.73(0.11)$ & 222 & $<-4.0$ \\
\hline SDSS J134226.93+052248.6 & 854 & 52373 & 371 & $7960(110)$ & $-8.97(0.10)$ & 146 & $<-4.0$ \\
\hline SDSS J134459.35+650512.5 & 497 & 51989 & 124 & $10010(450)$ & $-9.65(0.20)$ & 320 & $<-5.0$ \\
\hline SDSS J135118.47+425316.0 & 1345 & 52814 & 32 & $6770(120)$ & $-11.35(0.08)$ & 45 & $<-3.0$ \\
\hline SDSS J135137.07+613607.0 & 786 & 52319 & 571 & $7190(150)$ & $-10.26(0.14)$ & 166 & $<-3.0$ \\
\hline SDSS J135637.78+404703.4 & 1378 & 53061 & 444 & $7100(70)$ & $-8.89(0.05)$ & 125 & $<-3.0$ \\
\hline SDSS J140316.91-002450.0 & 301 & 51942 & 30 & $4660(110)$ & $-10.92(0.08)$ & 67 & $<-4.0$ \\
\hline SDSS J140445.10-023237.2 & 915 & 52443 & 114 & $7620(150)$ & $-9.36(0.11)$ & 143 & $<-4.0$ \\
\hline SDSS J141426.55-011354.7 & 303 & 51615 & 124 & $8940(200)$ & $-10.75(0.06)$ & 109 & $<-5.0$ \\
\hline SDSS J142516.43-005048.7 & 305 & 51613 & 197 & $7220(90)$ & $-11.42(0.11)$ & 81 & $<-4.0$ \\
\hline SDSS J142931.17+583927.9 & 789 & 52342 & 93 & $9220(310)$ & $-8.52(0.15)$ & 235 & $<-4.0$ \\
\hline SDSS J143235.82+035423.3 & 585 & 52027 & 143 & $10760(430)$ & $-8.91(0.13)$ & 233 & $-4.35(0.18)$ \\
\hline SDSS J144022.52-023222.2 & 919 & 52409 & 36 & $6860(80)$ & $-10.44(0.20)$ & 100 & $-3.25(0.50)^{\mathrm{a}}$ \\
\hline
\end{tabular}


Table 2-Continued

\begin{tabular}{|c|c|c|c|c|c|c|c|}
\hline Name & Plate & MJD & Fiber & $T_{\text {eff }}(\mathrm{K})$ & $\log (\mathrm{Ca} / \mathrm{He})$ & $D(\mathrm{pc})$ & $\log (\mathrm{H} / \mathrm{He})$ \\
\hline SDSS J144453.67+574147.2 & 791 & 52435 & 419 & $9120(400)$ & $-7.44(0.36)$ & 360 & $<-4.0$ \\
\hline SDSS J144516.24-020849.6 & 920 & 52411 & 197 & $6530(80)$ & $-10.71(0.17)$ & 94 & $-3.53(0.58)^{\mathrm{a}}$ \\
\hline SDSS J151054.84+381450.0 & 1399 & 53172 & 198 & $9130(240)$ & $-8.55(0.13)$ & 229 & $-4.17(0.26)$ \\
\hline SDSS J151441.87+501209.7 & 1330 & 52822 & 533 & $9930(510)$ & $-9.40(0.36)$ & 377 & $<-4.0$ \\
\hline SDSS J153129.26+424015.7 & 1052 & 52466 & 291 & $7130(80)$ & $-10.36(0.04)$ & 88 & $<-5.0$ \\
\hline SDSS J154025.18+514921.6 & 796 & 52401 & 397 & $8070(250)$ & $-9.45(0.17)$ & 238 & $<-4.0$ \\
\hline SDSS J154540.22+492145.3 & 796 & 52401 & 77 & $9130(510)$ & $-8.79(0.35)$ & 334 & $<-4.0$ \\
\hline SDSS J154623.07+392723.6 & 1680 & 53171 & 588 & $8680(220)$ & $-8.92(0.10)$ & 192 & $<-5.0$ \\
\hline SDSS J154953.26+023929.7 & 594 & 52045 & 65 & $7990(380)$ & $-9.07(0.31)$ & 286 & $<-3.0$ \\
\hline SDSS J155852.59+031242.9 & 595 & 52023 & 484 & $7170(110)$ & $-9.30(0.08)$ & 136 & $<-4.0$ \\
\hline SDSS J161801.34+445220.8 & 814 & 52443 & 152 & $9560(290)$ & $-7.98(0.11)$ & 226 & $<-5.0$ \\
\hline SDSS J164026.65+315453.9 & 1340 & 52781 & 484 & $6840(100)$ & $-9.89(0.08)$ & 110 & $<-3.0$ \\
\hline SDSS J165741.59+373824.2 & 820 & 52433 & 443 & $7650(160)$ & $-9.79(0.11)$ & 182 & $<-4.0$ \\
\hline SDSS J165741.59+373824.2 & 820 & 52438 & 448 & $7660(160)$ & $-9.87(0.10)$ & 182 & $<-4.0$ \\
\hline SDSS J171139.82+220152.9 & 1689 & 53177 & 33 & $9160(350)$ & $-8.93(0.16)$ & 286 & $<-4.0$ \\
\hline SDSS J171436.43+550112.4 & 367 & 51997 & 304 & $9190(510)$ & $-9.52(0.23)$ & 377 & $<-4.0$ \\
\hline SDSS J171513.64+280403.8 & 980 & 52431 & 351 & $8240(150)$ & $-10.83(0.11)$ & 139 & $<-4.0$ \\
\hline SDSS J171900.63+562350.5 & 367 & 51997 & 402 & $9300(210)$ & $-9.29(0.05)$ & 192 & $<-5.0$ \\
\hline SDSS J205059.11-011021.9 & 1115 & 52914 & 281 & $9560(290)$ & $-7.59(0.15)$ & 291 & $<-5.0$ \\
\hline SDSS J210303.47-010842.5 & 1114 & 53179 & 4 & $8140(170)$ & $-8.88(0.16)$ & 182 & $<-4.0$ \\
\hline SDSS J210733.93+005557.7 & 985 & 52431 & 100 & $7510(100)$ & $-10.19(0.10)$ & 124 & $-3.10(0.18)$ \\
\hline SDSS J212424.69-011452.5 & 987 & 52523 & 74 & $7690(110)$ & $-9.18(0.08)$ & 125 & $<-4.0$ \\
\hline SDSS J222802.05+120733.3 & 737 & 52518 & 9 & $6760(20)$ & $-9.96(0.02)$ & 34 & $<-4.0$ \\
\hline SDSS J223222.32+010920.7 & 376 & 52143 & 565 & $6900(140)$ & $-10.46(0.14)$ & 133 & $<-3.0$ \\
\hline SDSS J223841.05+010150.3 & 674 & 52201 & 531 & $7140(140)$ & $-10.30(0.25)$ & 132 & $-4.97(0.79)^{\mathrm{a}}$ \\
\hline SDSS J234245.90+000632.4 & 682 & 52525 & 27 & $8570(410)$ & $-9.15(0.15)$ & 316 & $-3.20(0.18)$ \\
\hline SDSS J235516.61+143136.1 & 749 & 52226 & 119 & $6940(110)$ & $-10.80(0.25)$ & 129 & $-3.67(0.89)^{\mathrm{a}}$ \\
\hline
\end{tabular}

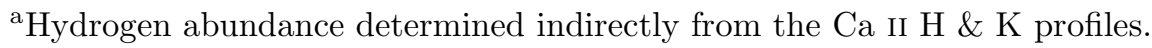


Fig. 1.- Representative synthetic spectra of DZ white dwarfs taken from our model grid at $\log g=8.0$ for various metal and hydrogen abundances. The spectra are normalized to unity at $4170 \AA$ and offset by an arbitrary factor for clarity. The labels indicate the effective temperature and the calcium abundance, $\log (\mathrm{Ca} / \mathrm{He})$, while the color of each spectrum corresponds to a different hydrogen abundance, as indicated in the lower right corner.

Fig. 2. - Fits to the Ca II H \& K lines with various hydrogen abundances for the DZ star G139-13 (WD 1705+030). [See the electronic version of the Journal for a color version of this figure.]

Fig. 3.- Fits to the Ca II H \& K lines with various hydrogen abundances for the DZ star G157-35 (WD 2312-024). [See the electronic version of the Journal for a color version of this figure.]

Fig. 4.- Fits to the energy distribution (left panels) and calcium lines (right panels) with helium-rich models including traces of metals and hydrogen (the abundances are indicated in each panel). The $B V R I$ and $J H K$ photometric observations are represented by error bars, while the average model fluxes are shown by filled circles. [See the electronic version of the Journal for a color version of this figure.]

Fig. 5.- Same as Fig. 4.

Fig. 6. - Fits to the energy distribution, calcium lines, and $\mathrm{H} \alpha$ profile (from left to right) for all DZ stars in our SDSS sample. The ugriz photometric observations are represented by error bars, while the average model fluxes are shown by filled circles. The atmospheric parameters are indicated in each panel. Since some of the spectroscopic observations have low signal-to-noise ratios, we have applied for clarity a three-point average window to the data displayed here. [See the electronic version of the Journal for a color version of this figure.]

Fig. 7.- Same as Fig. 6.

Fig. 8.- Same as Fig. 6.

Fig. 9.- Same as Fig. 6.

Fig. 10.- Same as Fig. 6.

Fig. 11. - Same as Fig. 6.

Fig. 12.- Same as Fig. 6. 
Fig. 13.- Same as Fig. 6.

Fig. 14.- Same as Fig. 6.

Fig. 15.- Same as Fig. 6.

Fig. 16. - Same as Fig. 6.

Fig. 17.- Same as Fig. 6.

Fig. 18. - Same as Fig. 6.

Fig. 19.- Same as Fig. 6.

Fig. 20.- Same as Fig. 6.

Fig. 21. - Same as Fig. 6.

Fig. 22.- Same as Fig. 6.

Fig. 23. - Same as Fig. 6.

Fig. 24. - Same as Fig. 6.

Fig. 25. - SDSS spectra with observed Ca II triplets compared with synthetic spectra interpolated at the atmospheric parameter solution obtained from the $\mathrm{Ca}$ II $\mathrm{H} \& \mathrm{~K}$ lines. [See the electronic version of the Journal for a color version of this figure.]

Fig. 26. - SDSS spectra of DZ stars showing the Mg I "b" blend at $\sim 5175 \AA$ compared with synthetic spectra interpolated at the atmospheric parameter solution obtained from the Ca II H \& K lines. The feature at $\sim 4860 \AA$ is $\mathrm{H} \beta$. [See the electronic version of the Journal for a color version of this figure.]

Fig. 27. - Relative abundance of calcium with respect to helium as a function of effective temperature for the BRL97/BLR01 and SDSS samples. The two continuous curves define the range of expected abundances for a low accretion rate in solar proportion of $5 \times 10^{-20} M_{\odot}$ $\mathrm{yr}^{-1}$ (lower curve) and a high rate of $5 \times 10^{-15} M_{\odot} \mathrm{yr}^{-1}$ (upper curve). The dashed line indicates the detection limit of the $\mathrm{Ca}$ II $\mathrm{H} \& \mathrm{~K}$ lines (taken as $5 \AA$ of total equivalent width). The error bars in the bottom left corner represent the average uncertainties of $T_{\text {eff }}$ $(\sim 240 \mathrm{~K})$ and $\log (\mathrm{Ca} / \mathrm{He})(\sim 0.15 \mathrm{dex})$. Filled symbols correspond to DZ stars with hydrogen abundances determined directly from $\mathrm{H} \alpha$ or indirectly from the Ca II lines (these are marked with an additional + sign), while open symbols correspond to objects with only upper limits. The approximate hydrogen abundance for each object (or upper limits) can 
be deduced from the number of sides of the polygon (triangles: $\log (\mathrm{H} / \mathrm{He}) \sim-3$, squares: $\log (\mathrm{H} / \mathrm{He}) \sim-4$, pentagons: $\log (\mathrm{H} / \mathrm{He}) \sim-5$; circles represent pure helium solutions $)$.

Fig. 28. - The $(g-r, u-g)$ color-color diagram. Small dots represent objects with stellar images while filled triangles correspond to the SDSS DZ stars from Table 2. The curves show our DZ photometric sequences at $\log g=8, \log (\mathrm{H} / \mathrm{He})=-30$, for $T_{\text {eff }}=12,000 \mathrm{~K}$ (bottom left parabola) down to $5000 \mathrm{~K}$ (upper right parabola) by steps of $500 \mathrm{~K}$, and for calcium abundances of $\log (\mathrm{Ca} / \mathrm{He})=-7$ (bottom sequence) down to -12 by steps of 0.5 dex. The uppermost sequence corresponds to the pure helium models.

Fig. 29.- Hydrogen-to-helium abundance ratios as a function of effective temperature for the BRL/BLR and SDSS samples. Filled circles correspond to objects with hydrogen abundance determinations; objects with abundances measured indirectly from the calcium lines are marked with an additional + sign. The crosses indicate white dwarfs with only upper limit determinations. Solid lines represent the expected abundances for continuous accretion of material from the ISM with a solar composition and accretion rates of $10^{-21}$ to $10^{-18} M_{\odot}$ $\mathrm{yr}^{-1}$.

Fig. 30.- Calcium-to-hydrogen abundance ratios as a function of effective temperature for the BRL/BLR and SDSS samples. The horizontal dashed line indicates the solar $\mathrm{Ca} / \mathrm{H}$ abundance ratio, while the solid line corresponds to the maximum value expected from accretion of material with solar compositions. The various symbols are explained in the caption of Figure 27.

Fig. 31. - Total mass of hydrogen present in the helium convection zone as a function of effective temperature. The symbols are the same as in Figure 29. The solid curves indicate the amount of hydrogen expected from continuous accretion of material with solar composition and accretion rates of $10^{-21}$ to $10^{-18} M_{\odot} \mathrm{yr}^{-1}$.

Fig. 32. - Location of the hydrogen convection zone (hatched area) as a function of $T_{\text {eff }}$ in a $0.6 M_{\odot}$ DA white dwarf. The y-axis expresses on a logarithmic scale the mass fraction $\Delta M$ above a certain point with respect to the total mass of the star $M_{\star}$. The thick solid line indicates the location of the photosphere. 


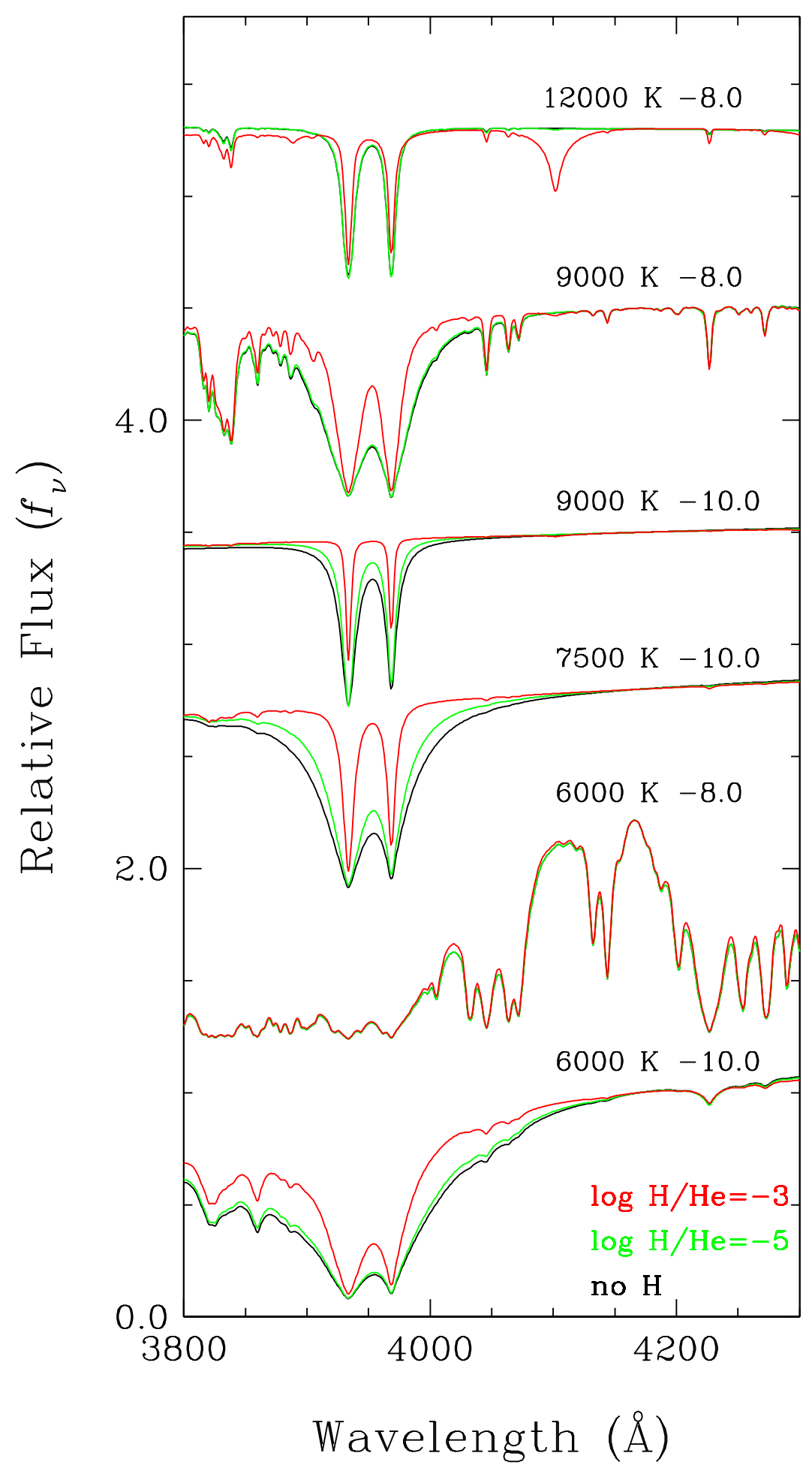

Figure 1 


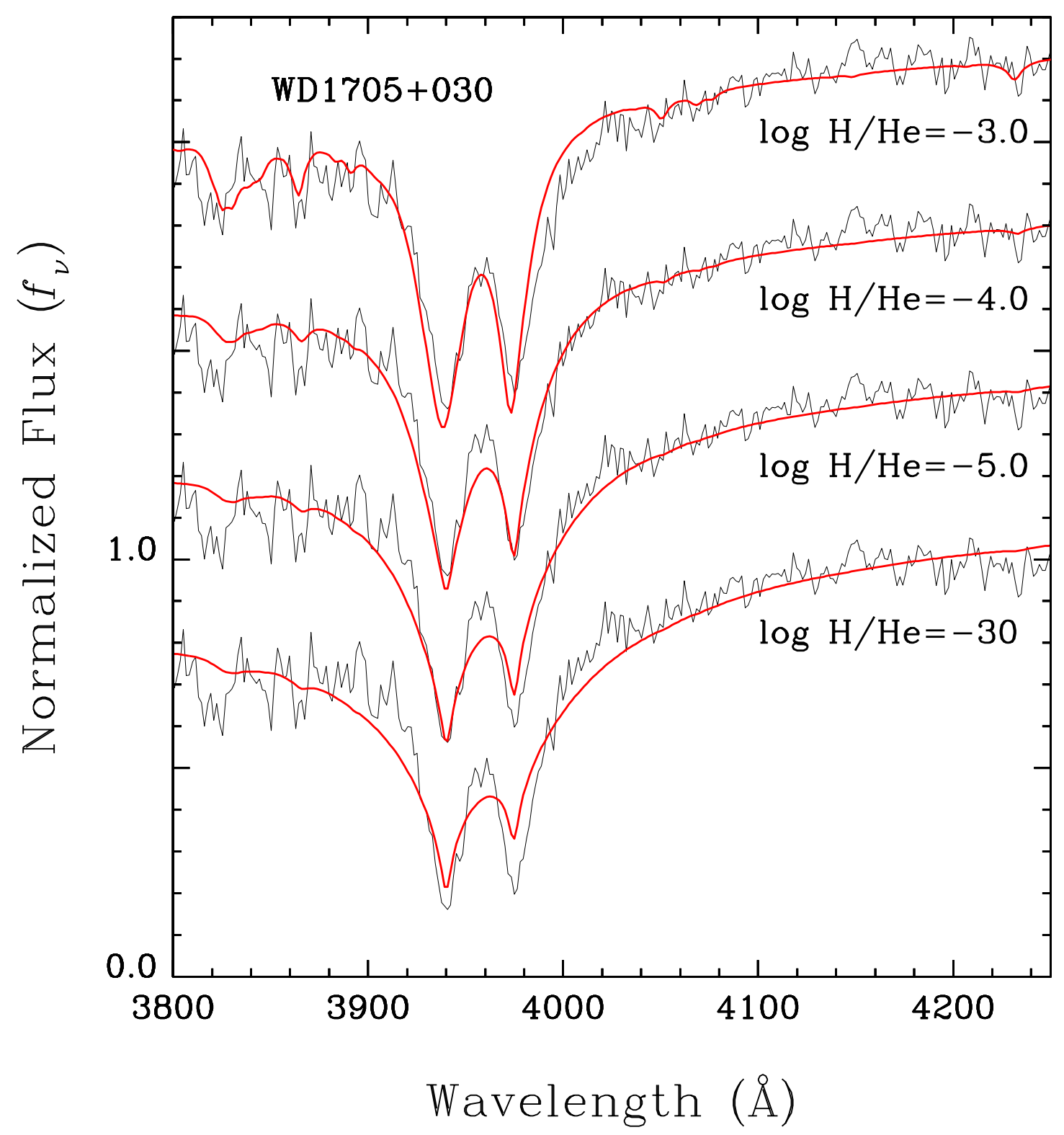

Figure 2 


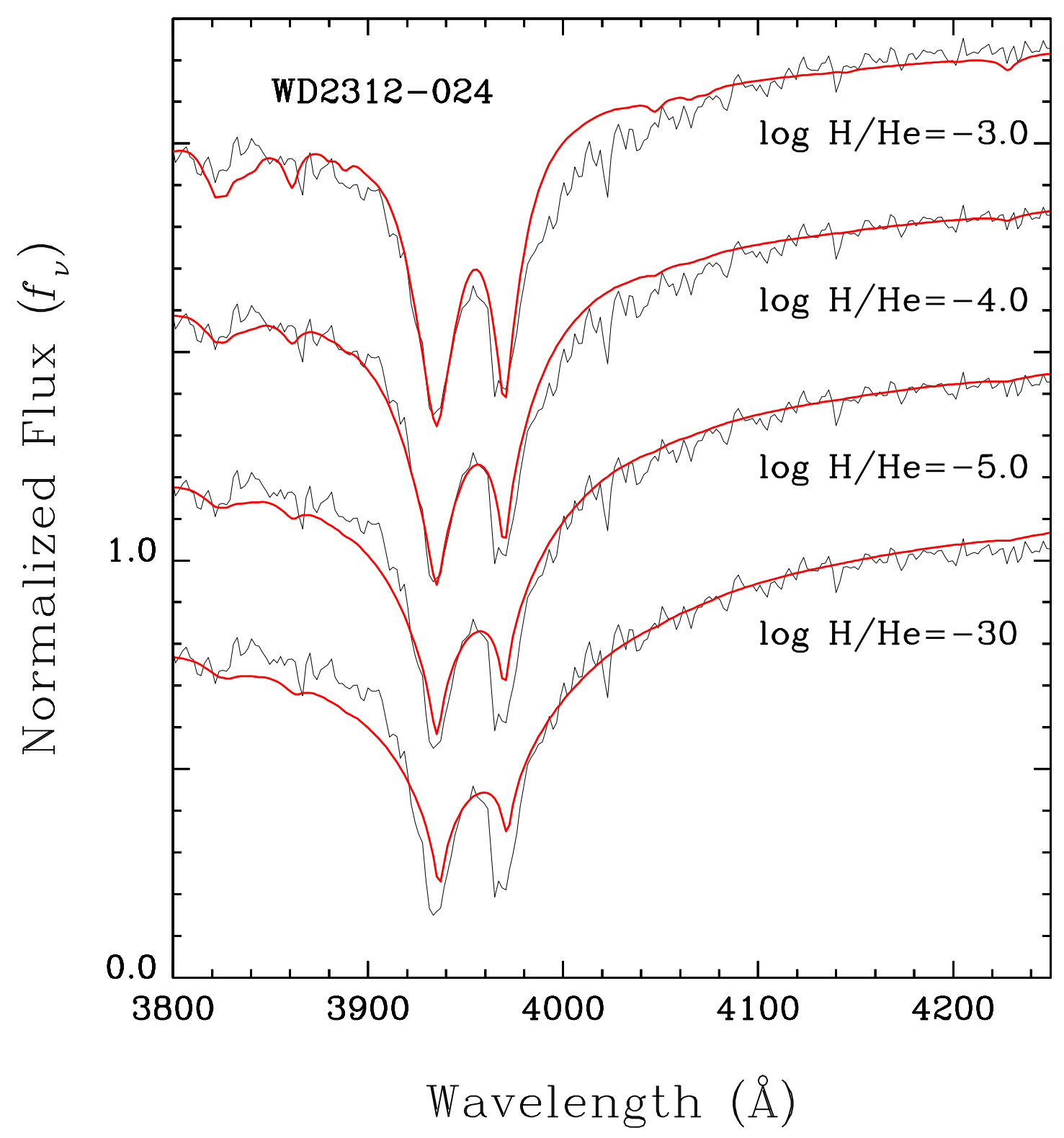

Figure 3 


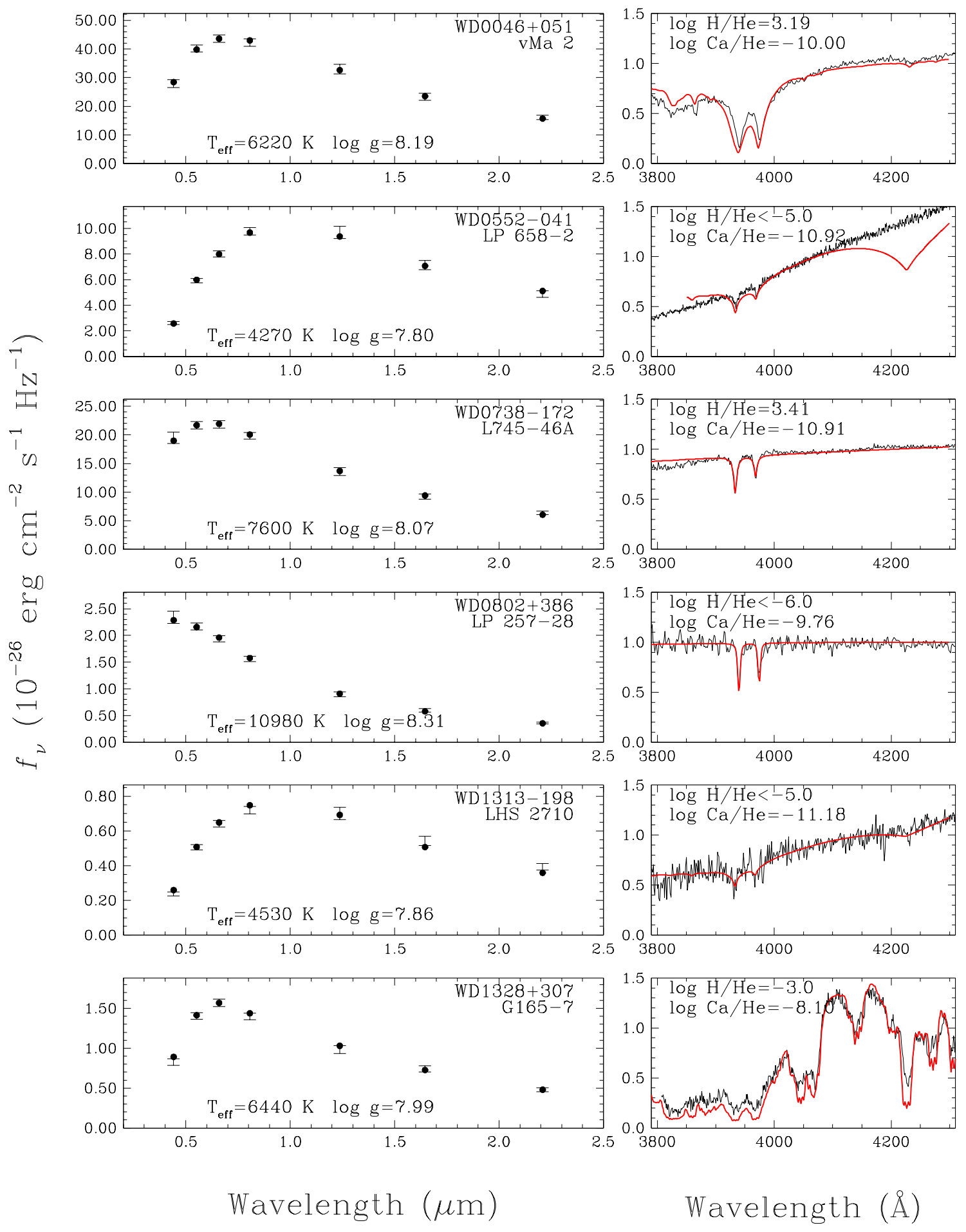

Figure 4 

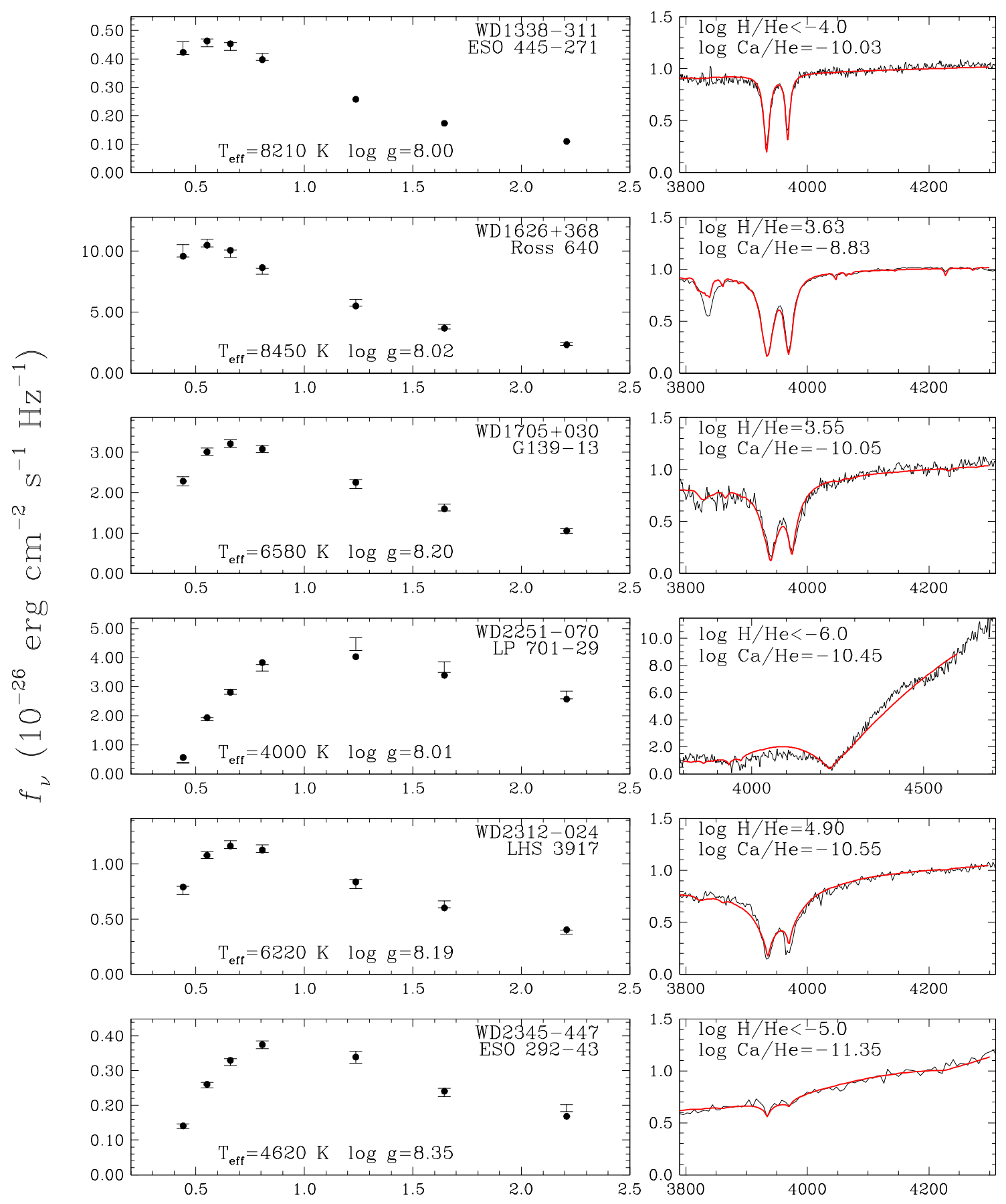

Wavelength $(\mu \mathrm{m})$

Wavelength $(\AA)$ 

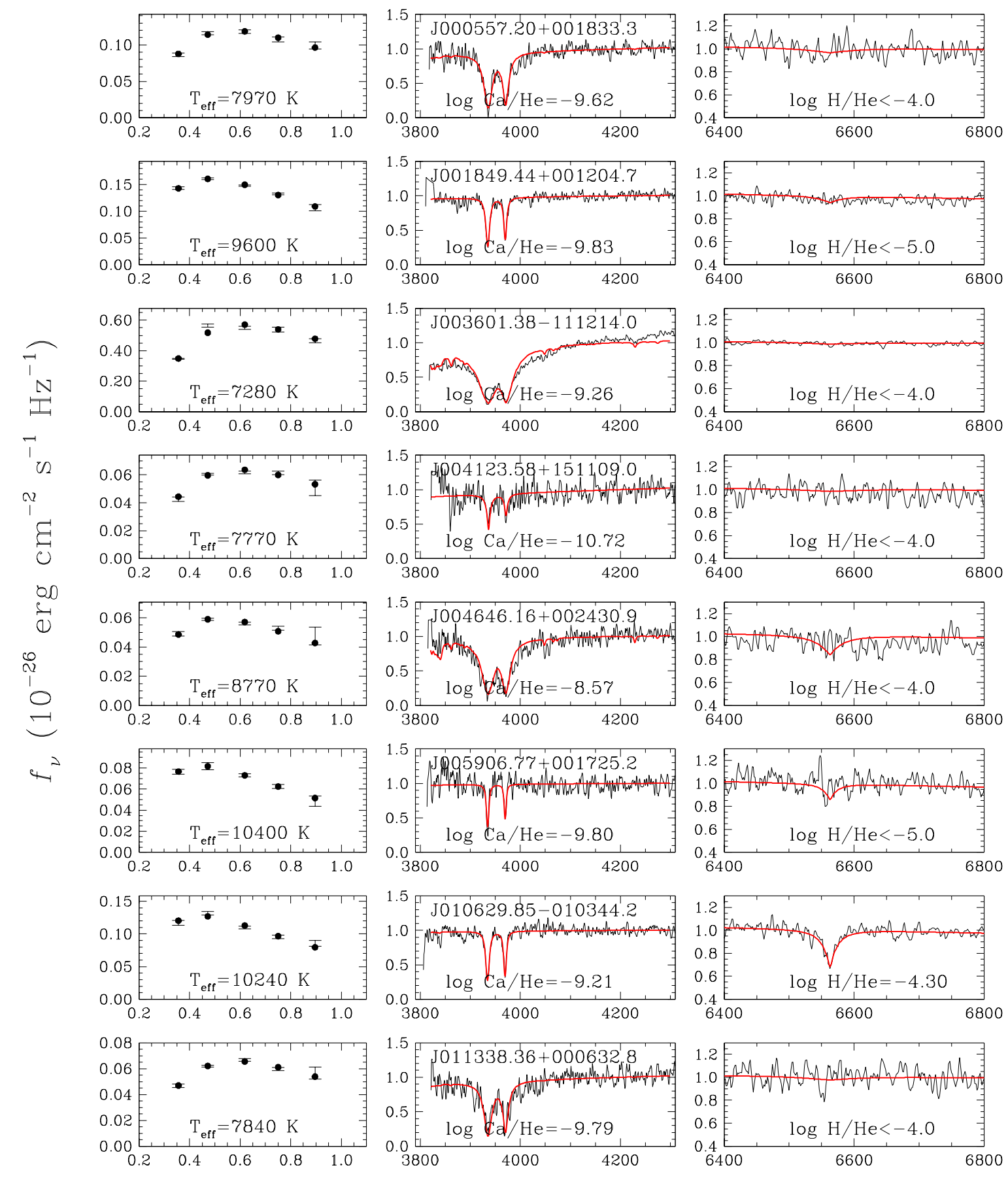

Wavelength ( $\mu \mathrm{m})$
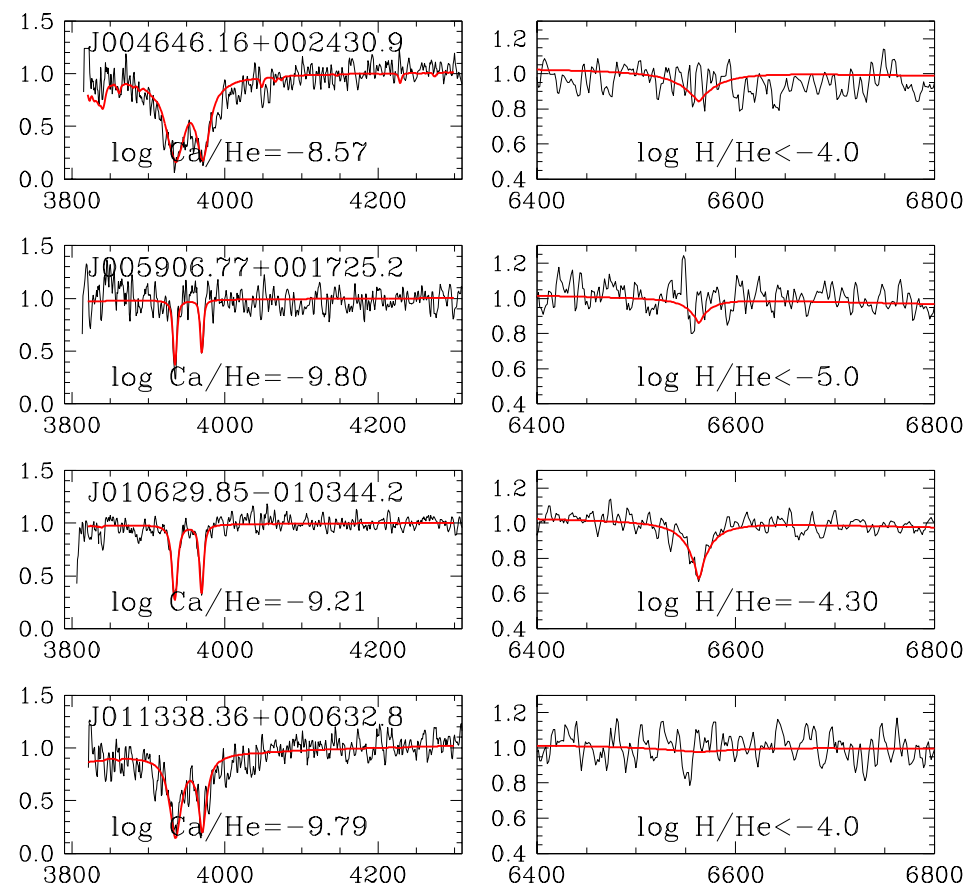

Wavelength $(\AA)$

Figure 6 

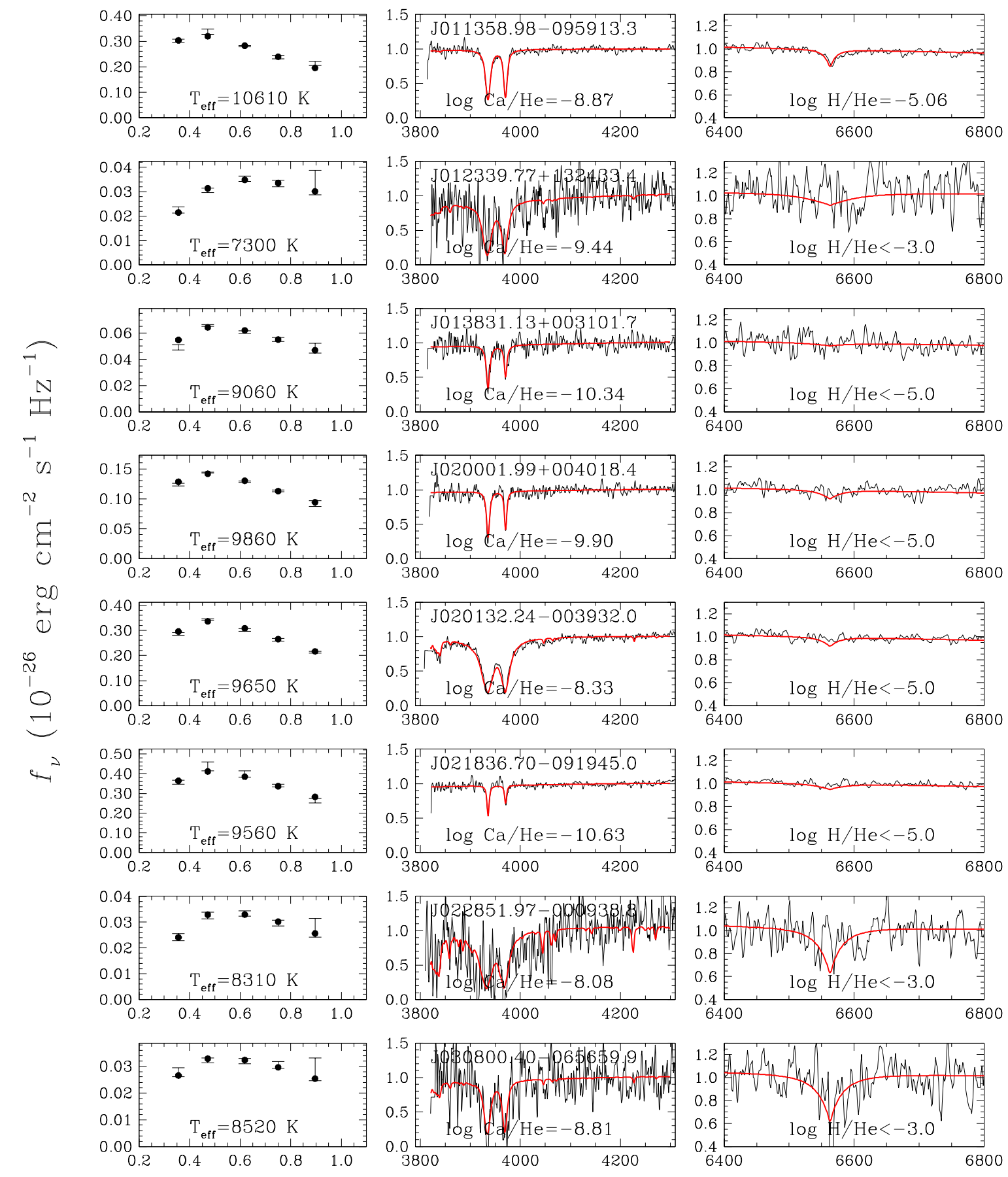

Wavelength ( $\mu \mathrm{m})$
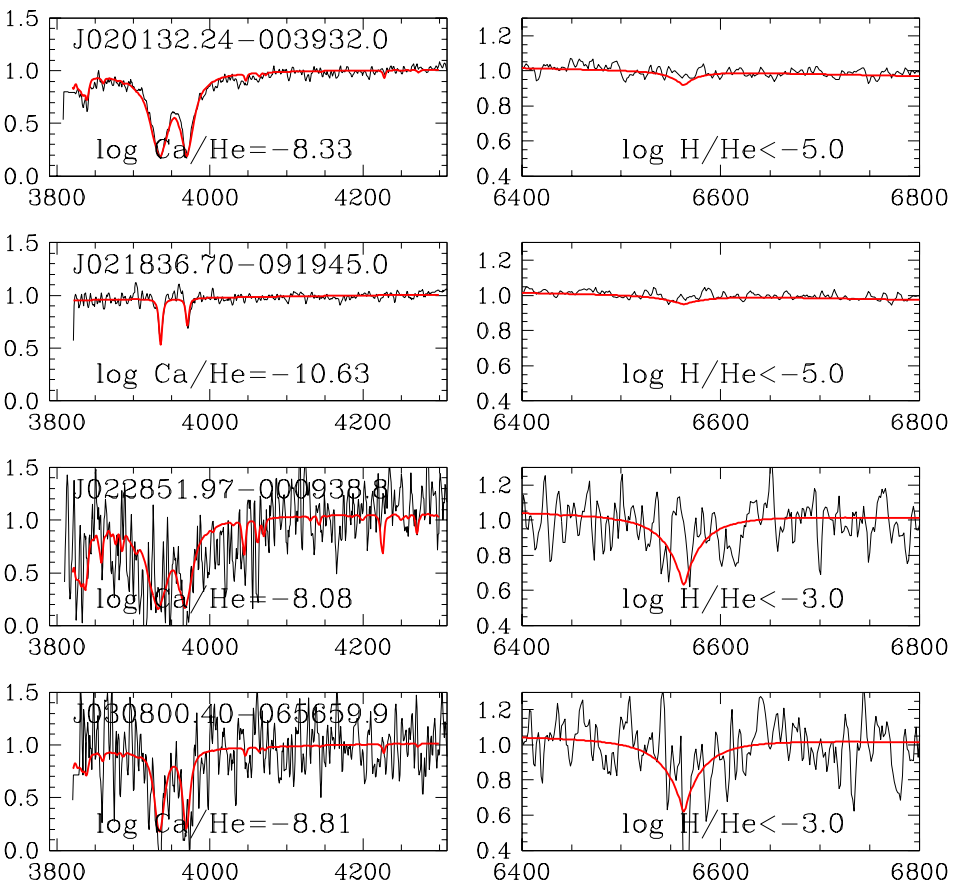

Wavelength $(\AA)$ 

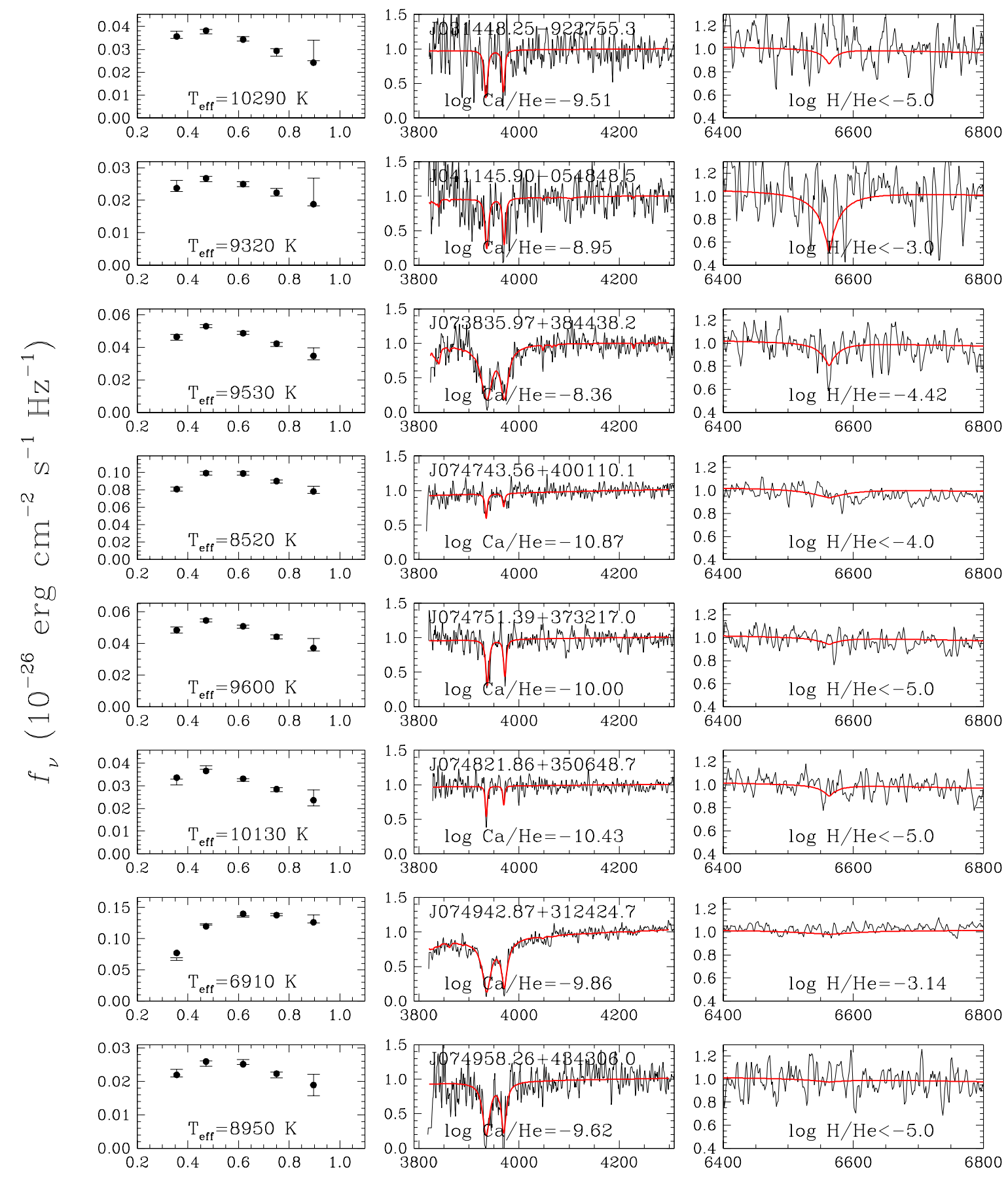

Wavelength ( $\mu \mathrm{m})$
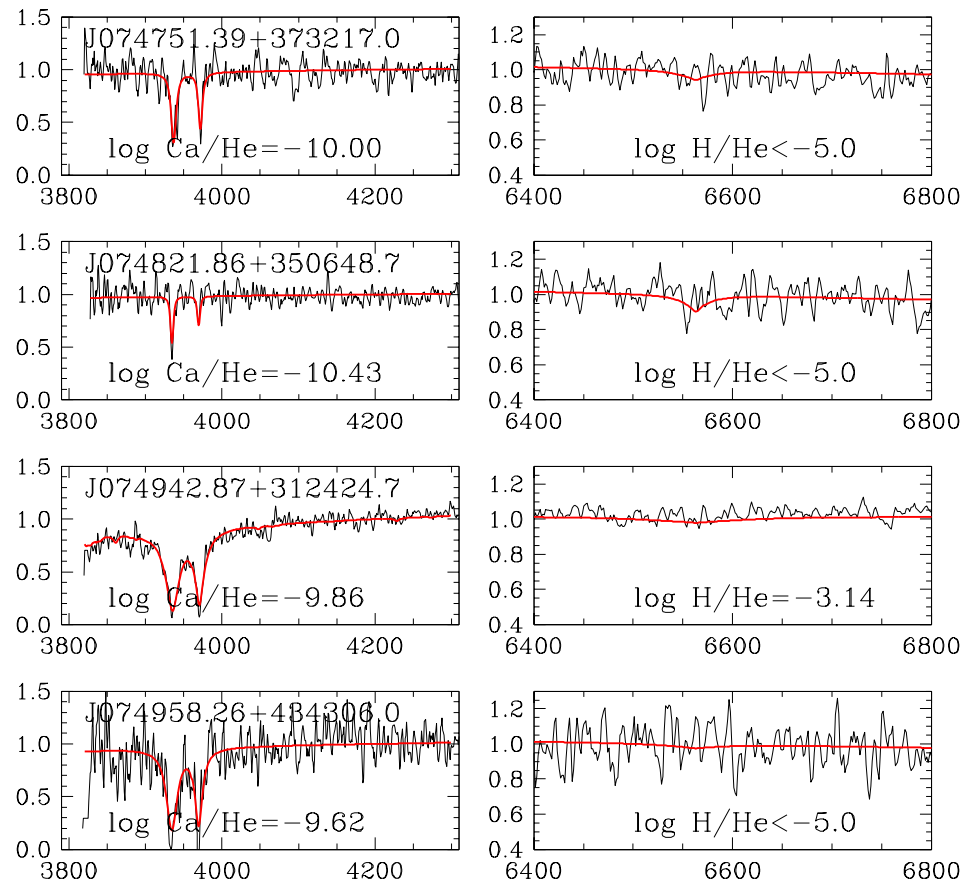

Wavelength $(\AA)$

Figure 8 

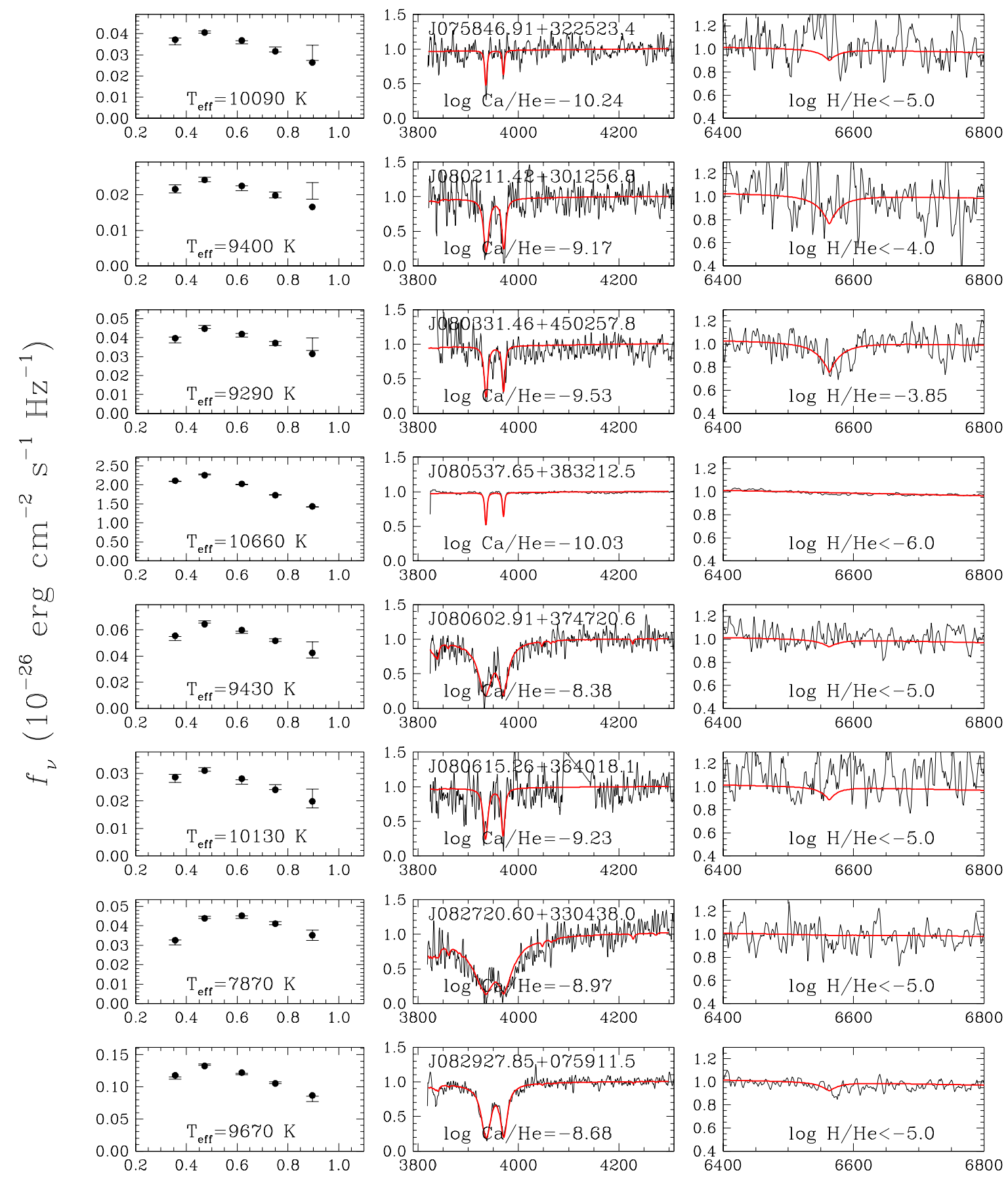

Wavelength ( $\mu \mathrm{m})$
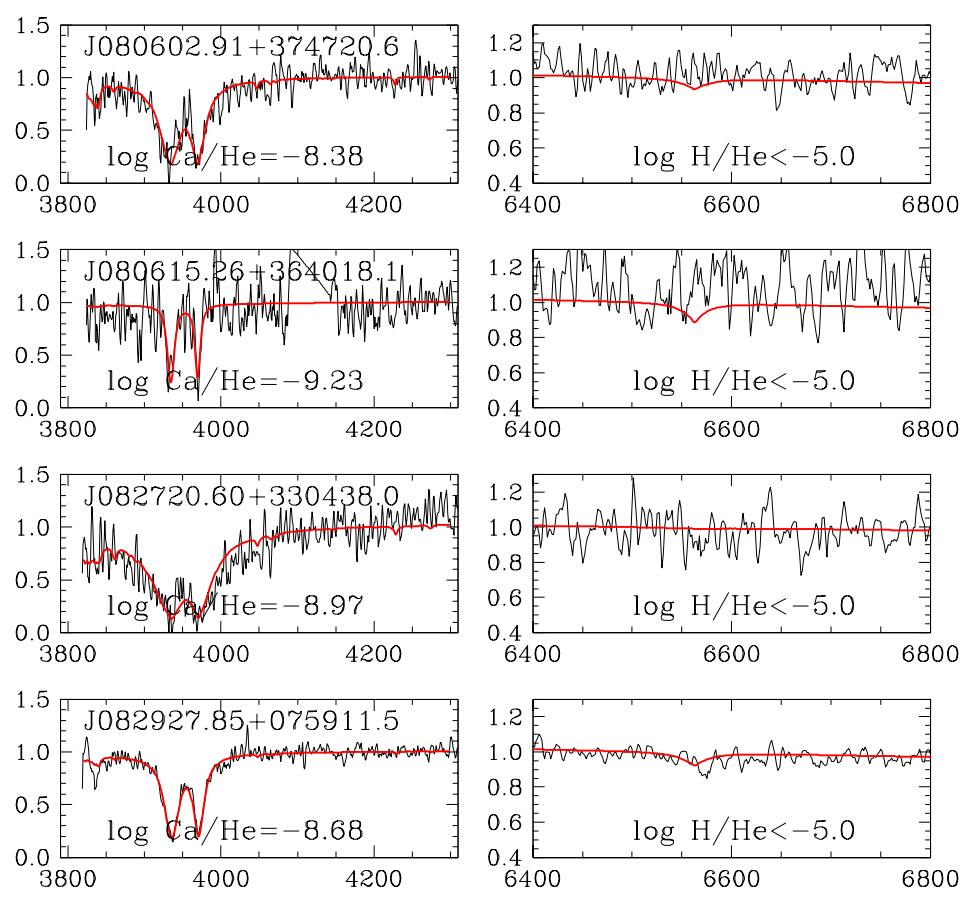

Wavelength $(\AA)$

Figure 9 

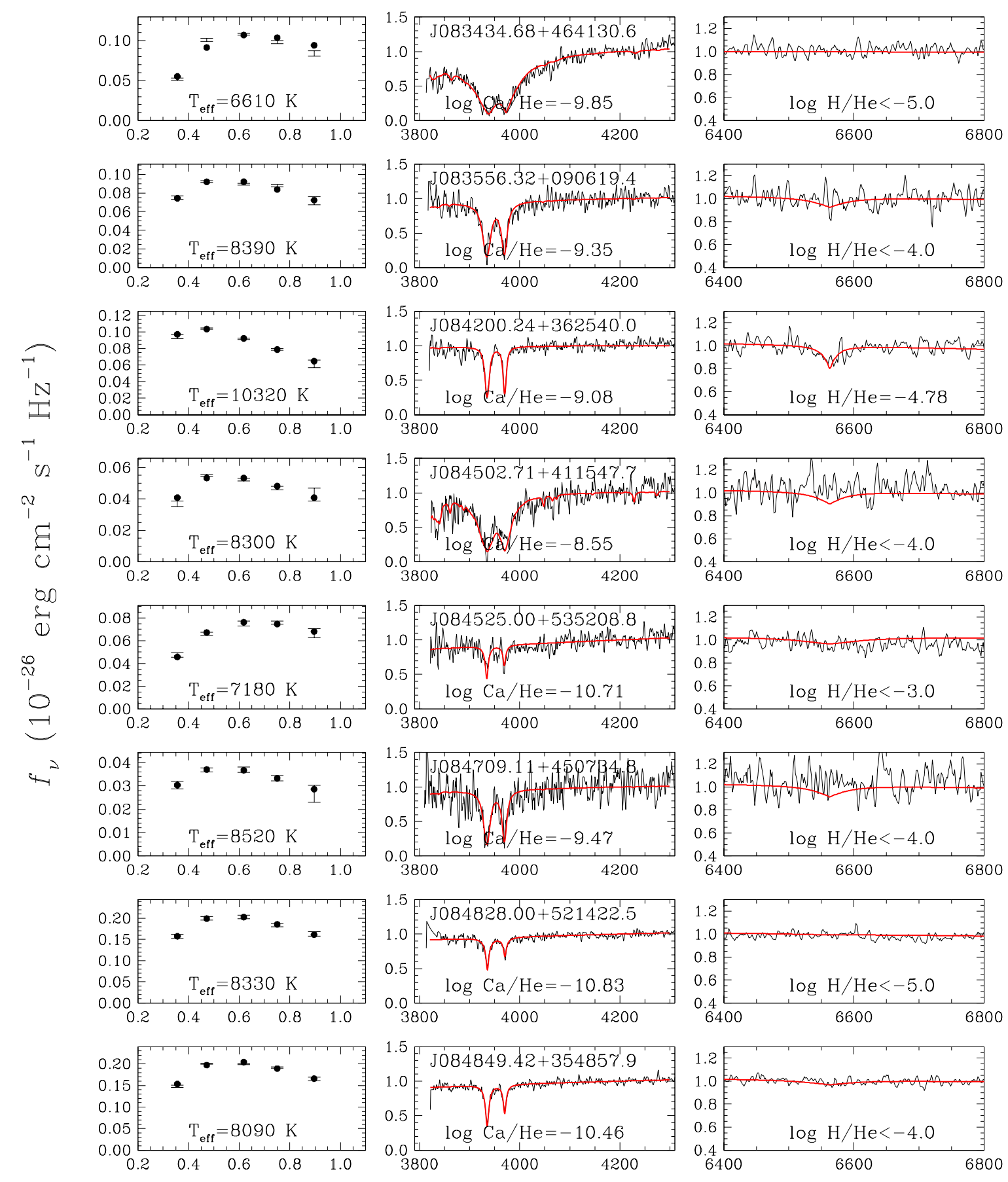

Wavelength ( $\mu \mathrm{m})$
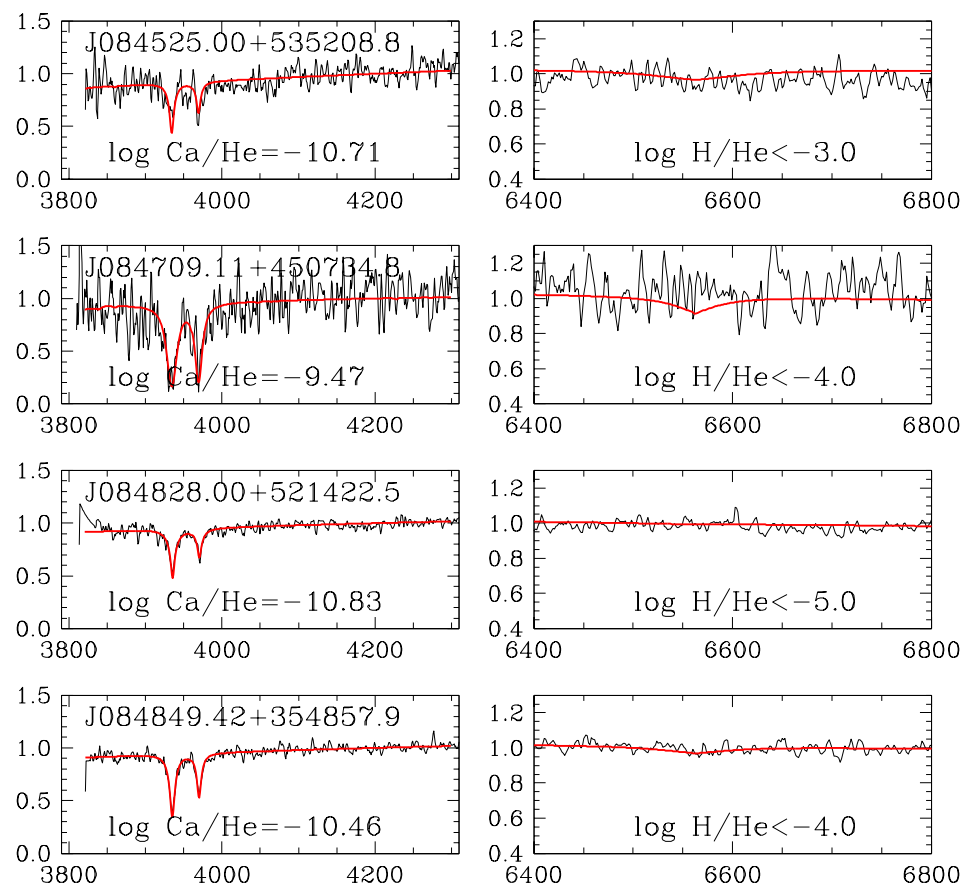

Wavelength $(\AA)$

Figure 10 

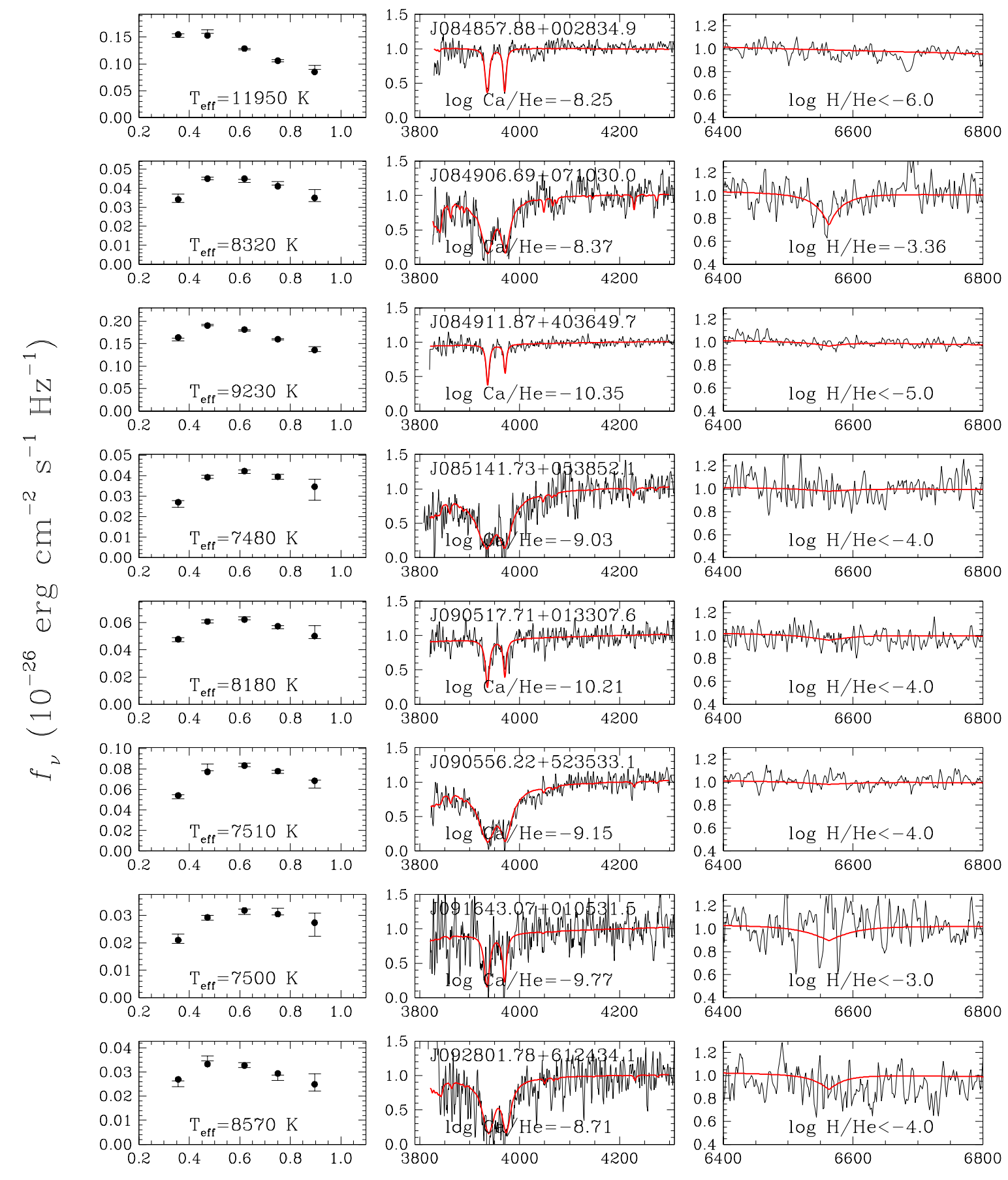

Wavelength ( $\mu \mathrm{m})$
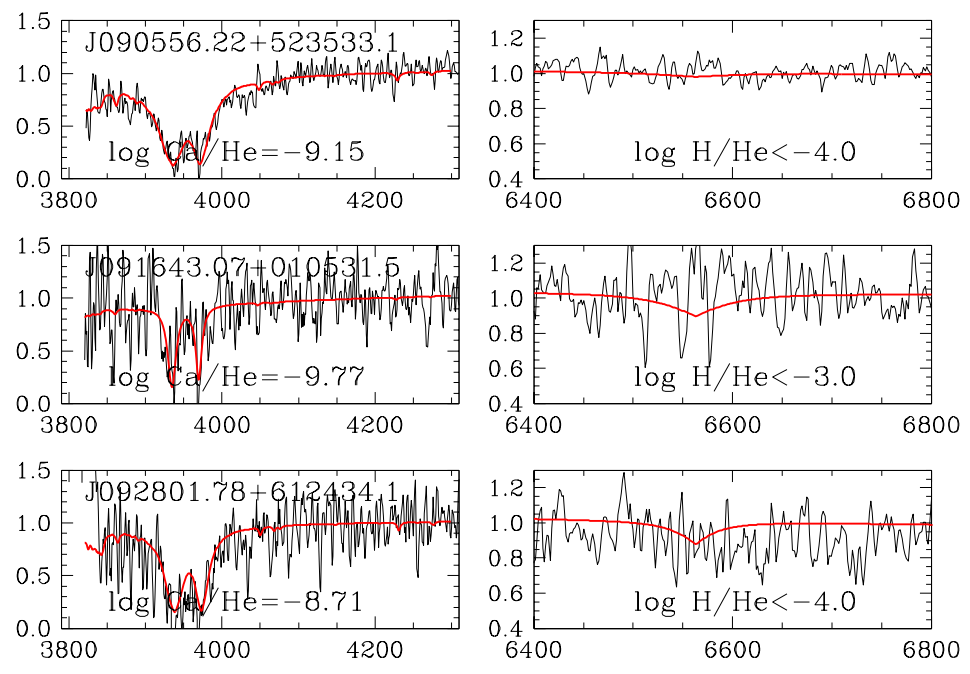

Wavelength $(\AA)$

Figure 11 

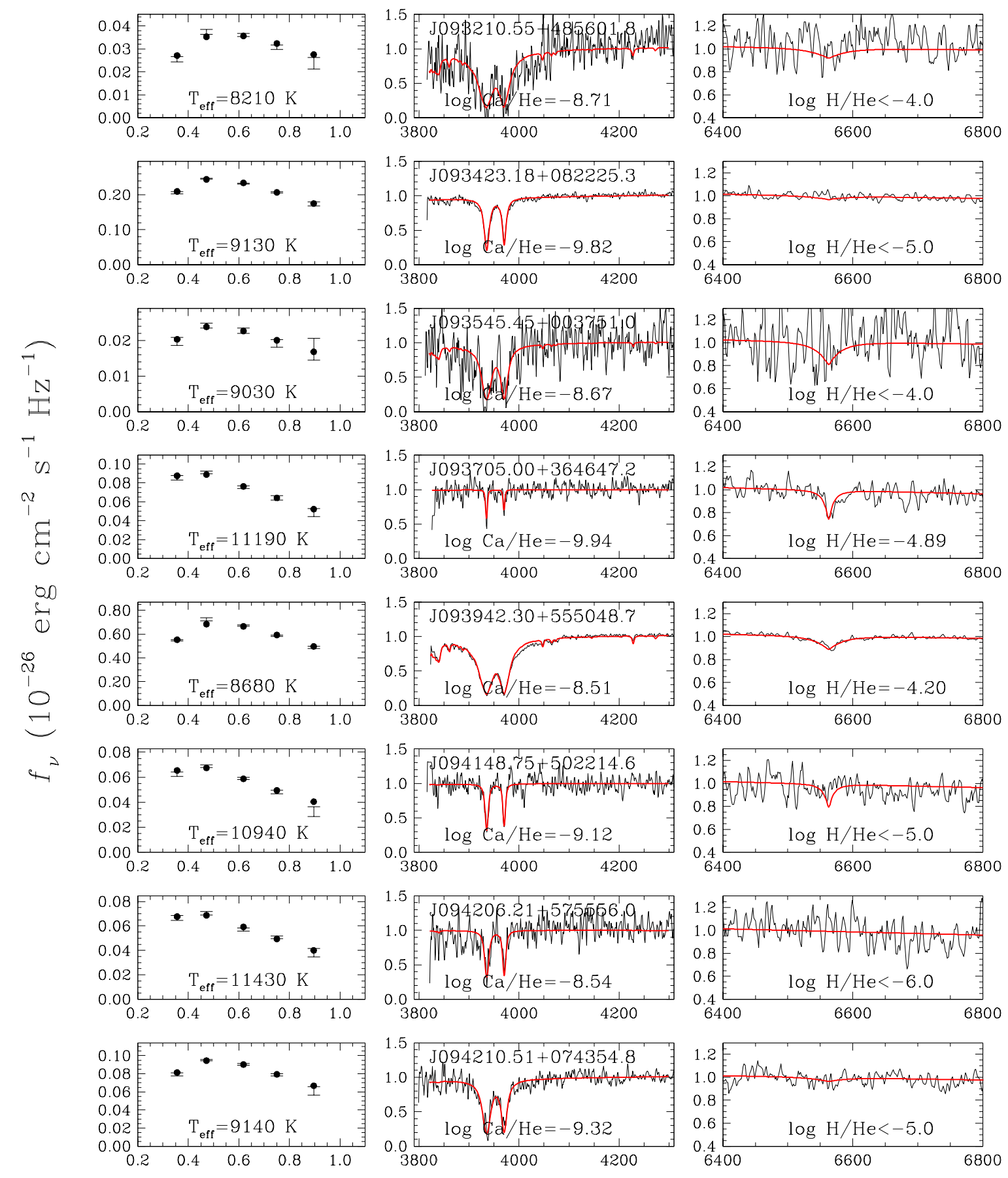

Wavelength ( $\mu \mathrm{m})$
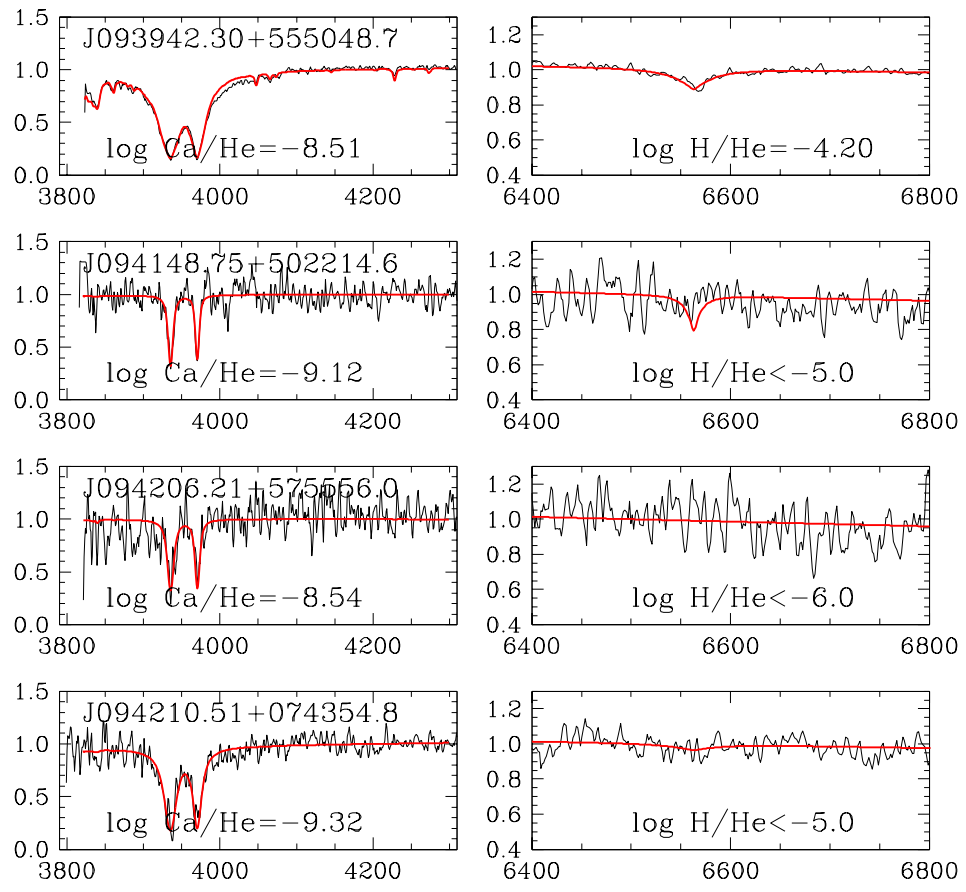

Wavelength $(\AA)$

Figure 12 

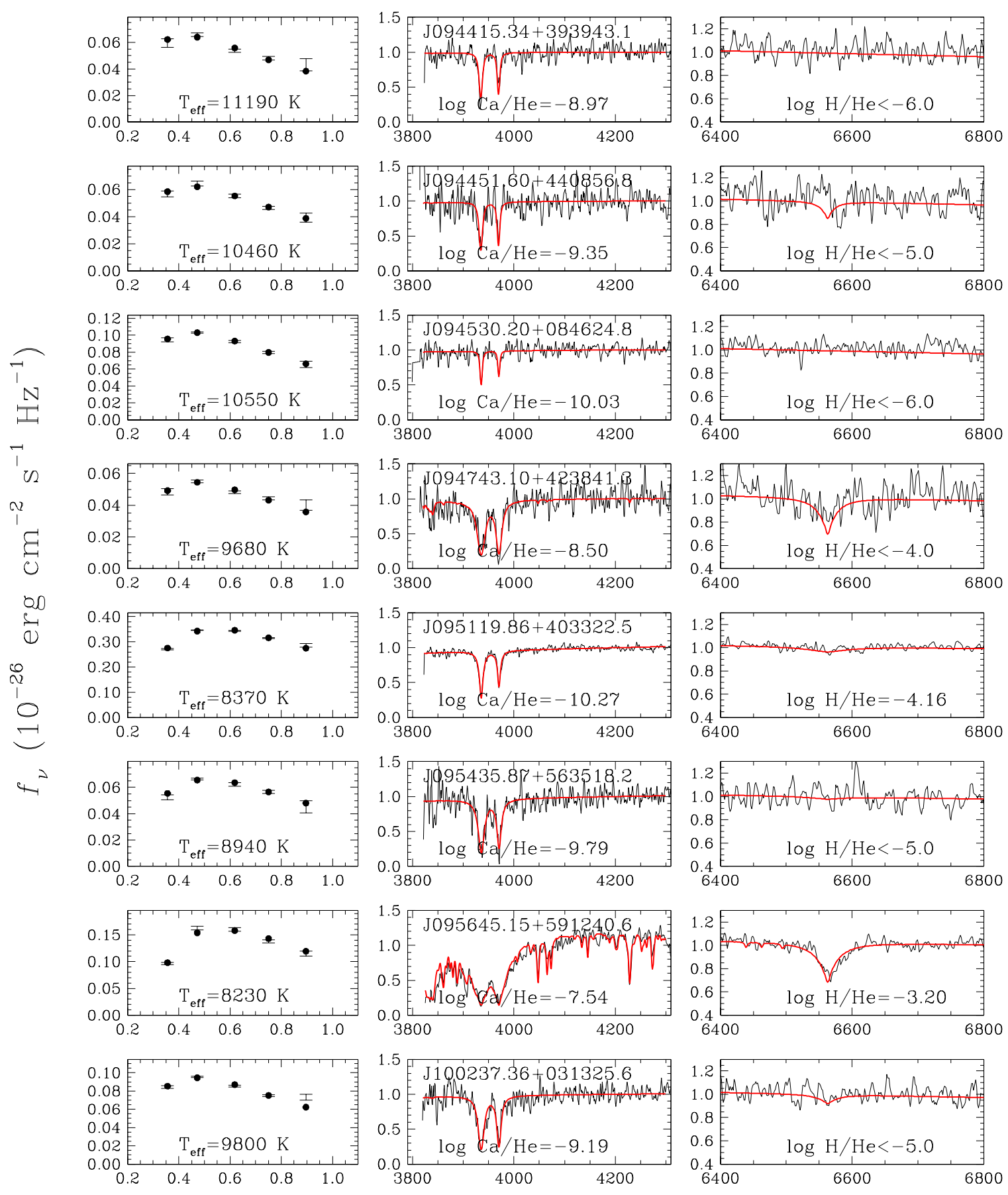

Wavelength $(\mu \mathrm{m})$

Wavelength $(\AA)$

Figure 13 

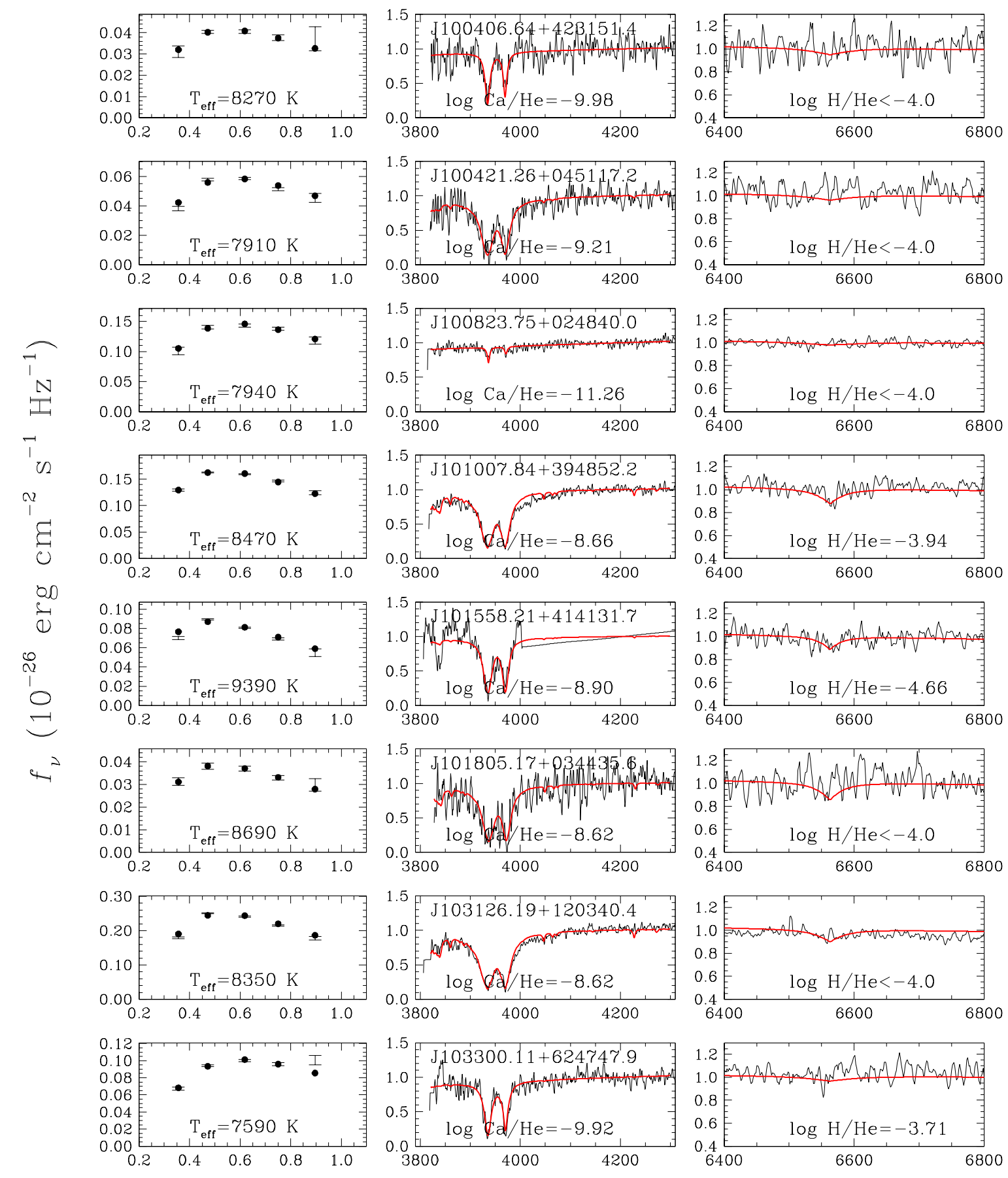

Wavelength ( $\mu \mathrm{m})$
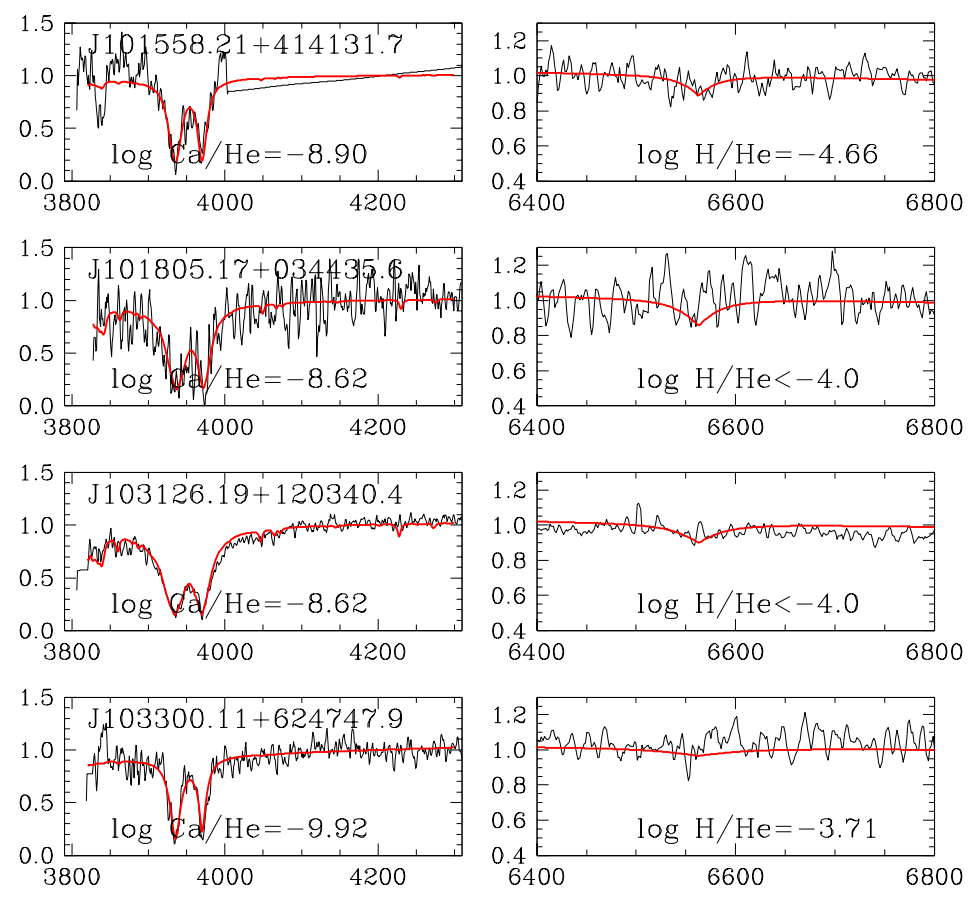

Wavelength $(\AA)$

Figure 14 

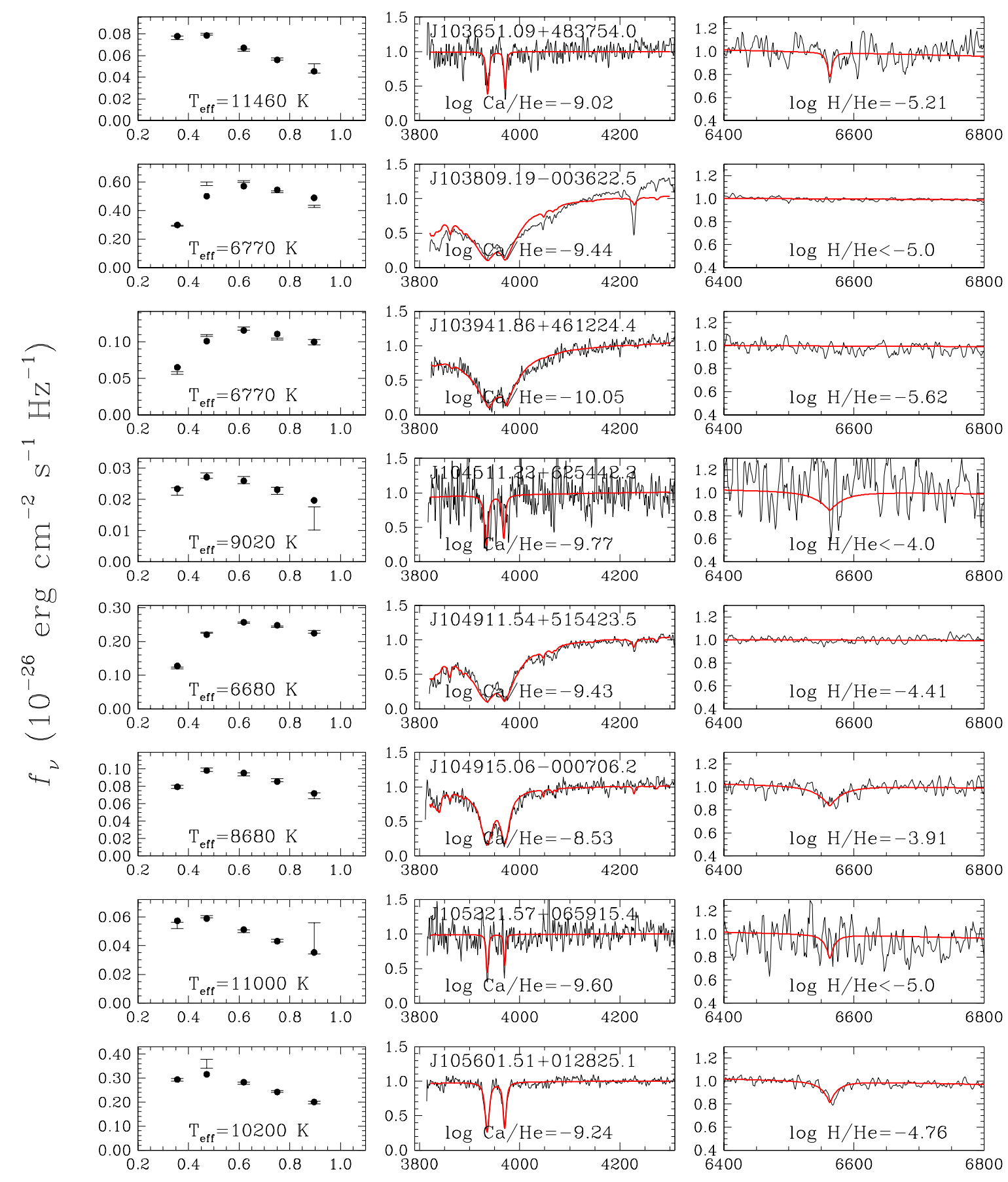

Wavelength ( $\mu \mathrm{m})$
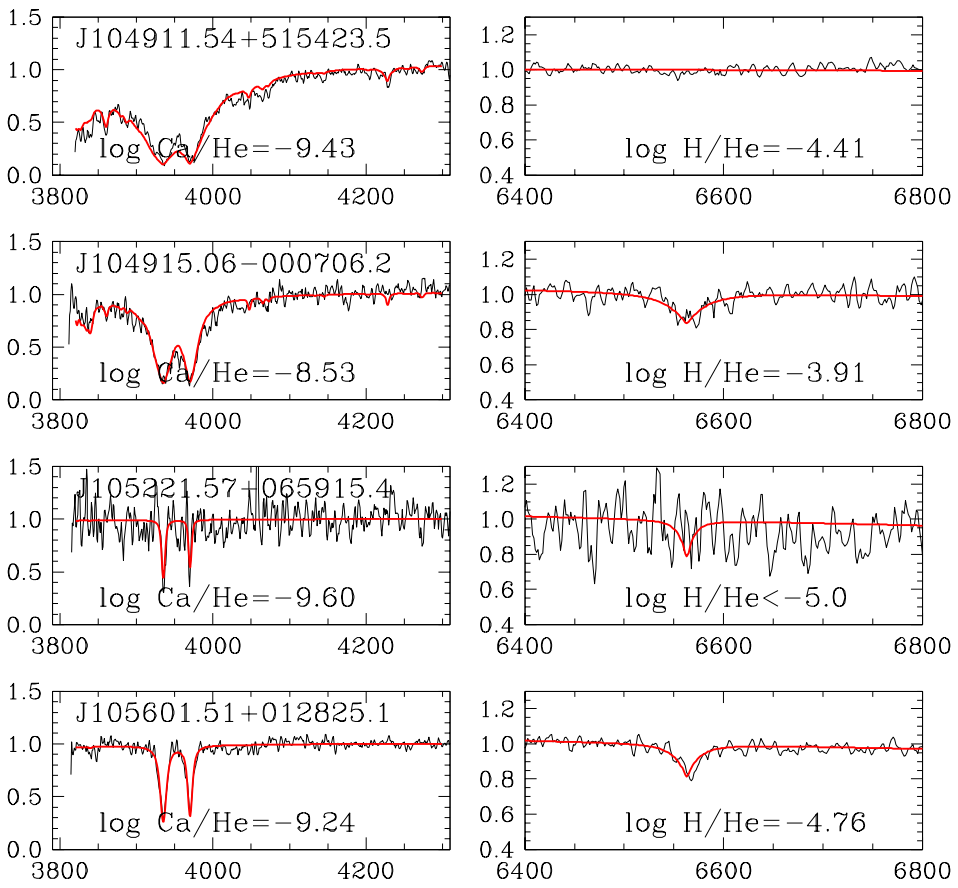

Wavelength $(\AA)$ 

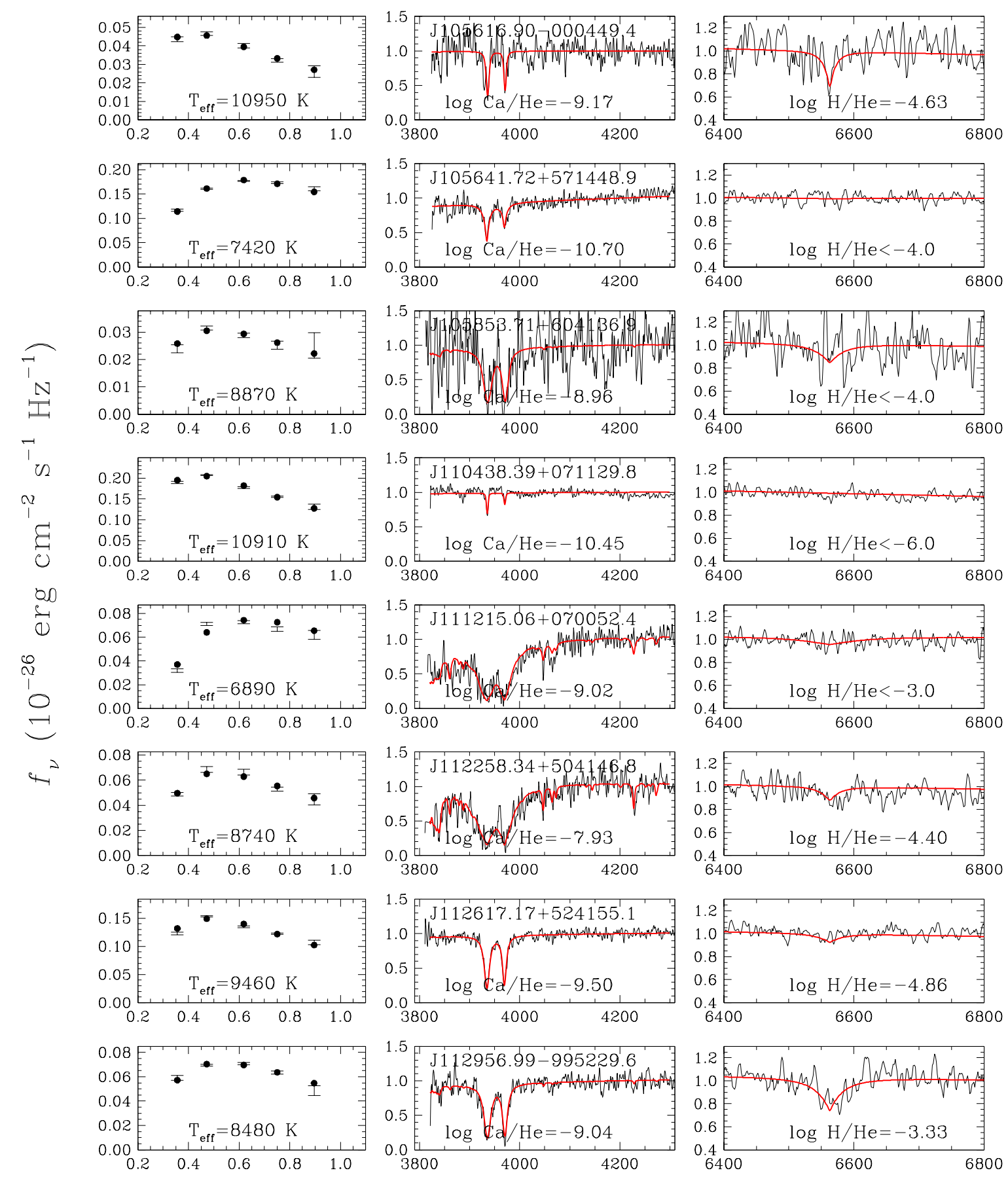

Wavelength ( $\mu \mathrm{m})$
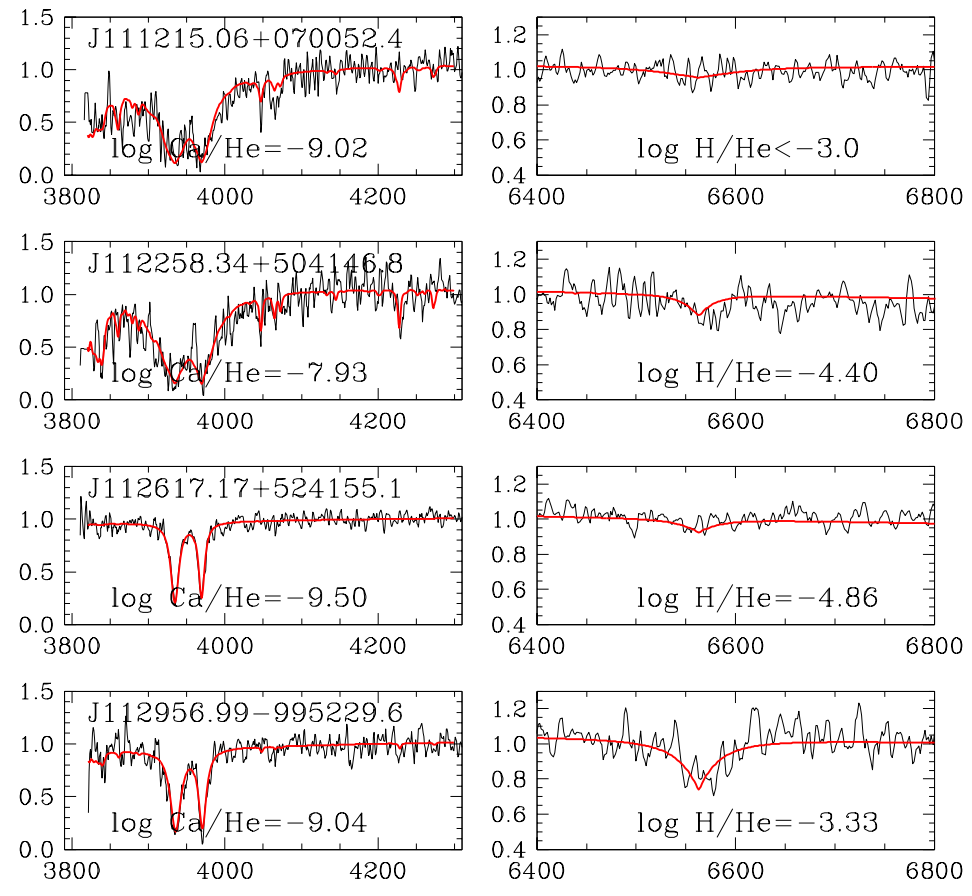

Wavelength $(\AA)$

Figure 16 

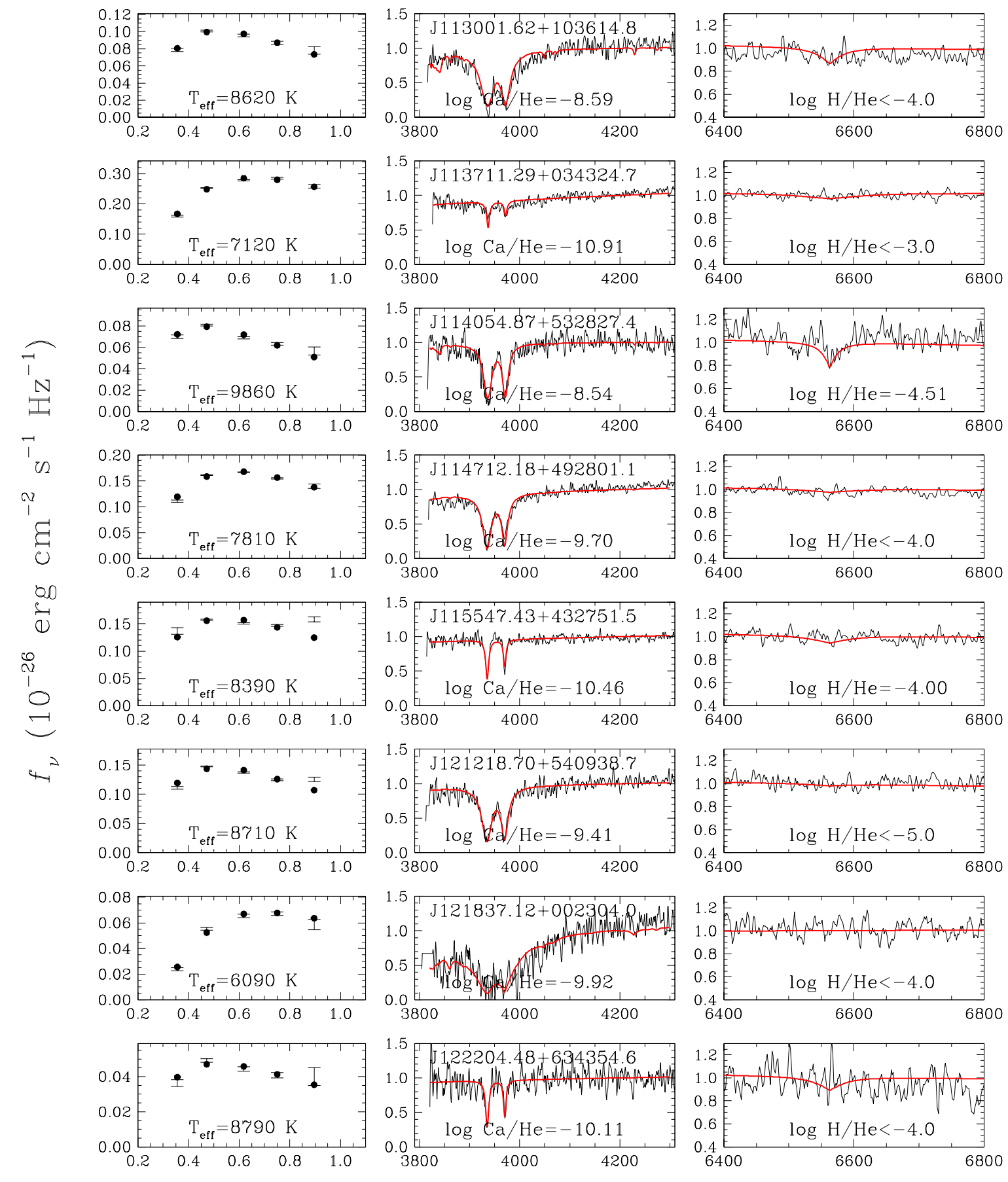

Wavelength ( $\mu \mathrm{m})$
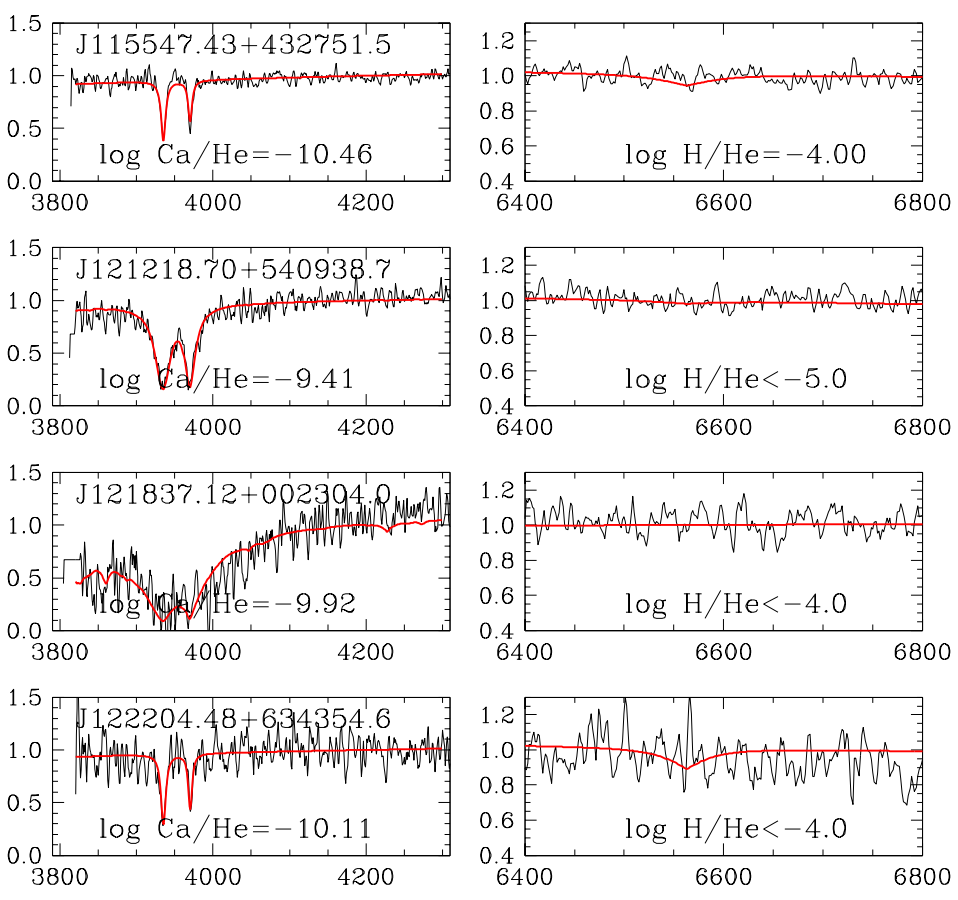

Wavelength $(\AA)$

Figure 17 

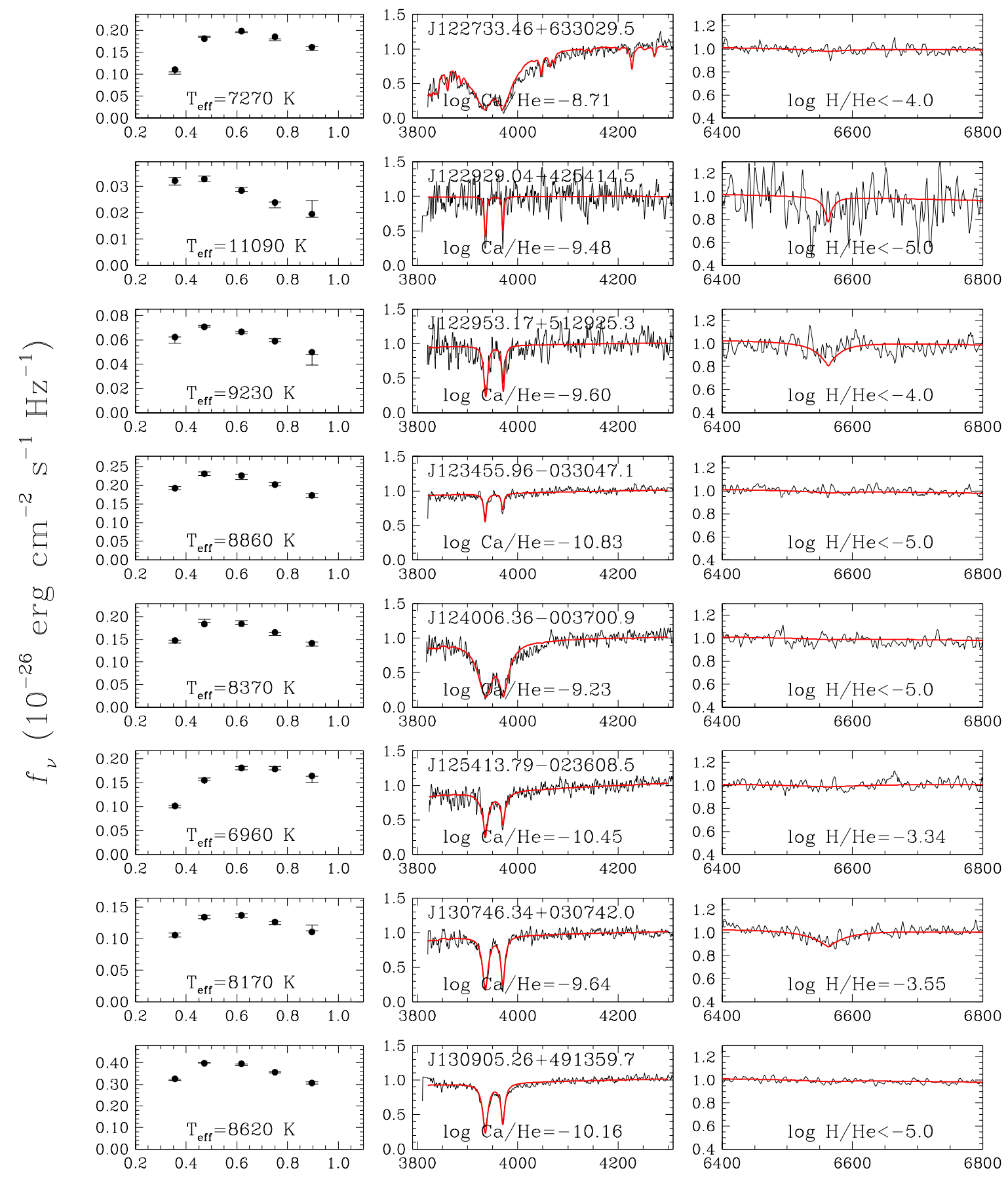

Wavelength $(\mu \mathrm{m})$
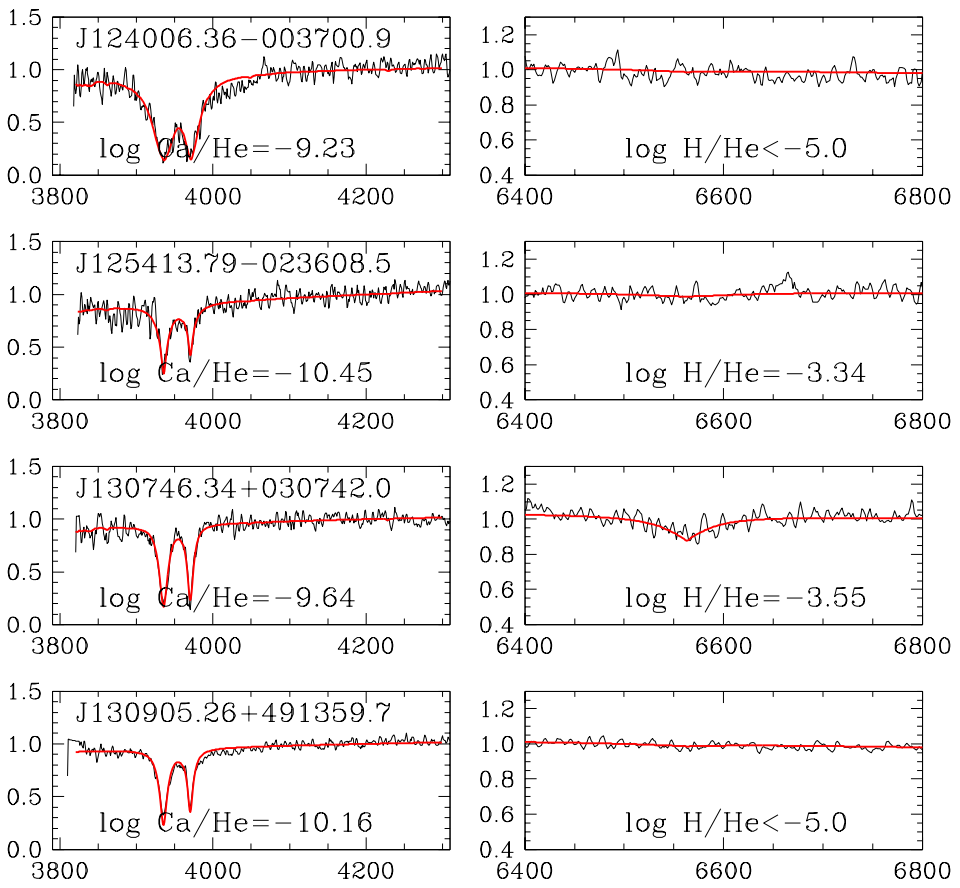

Wavelength $(\AA)$

Figure 18 

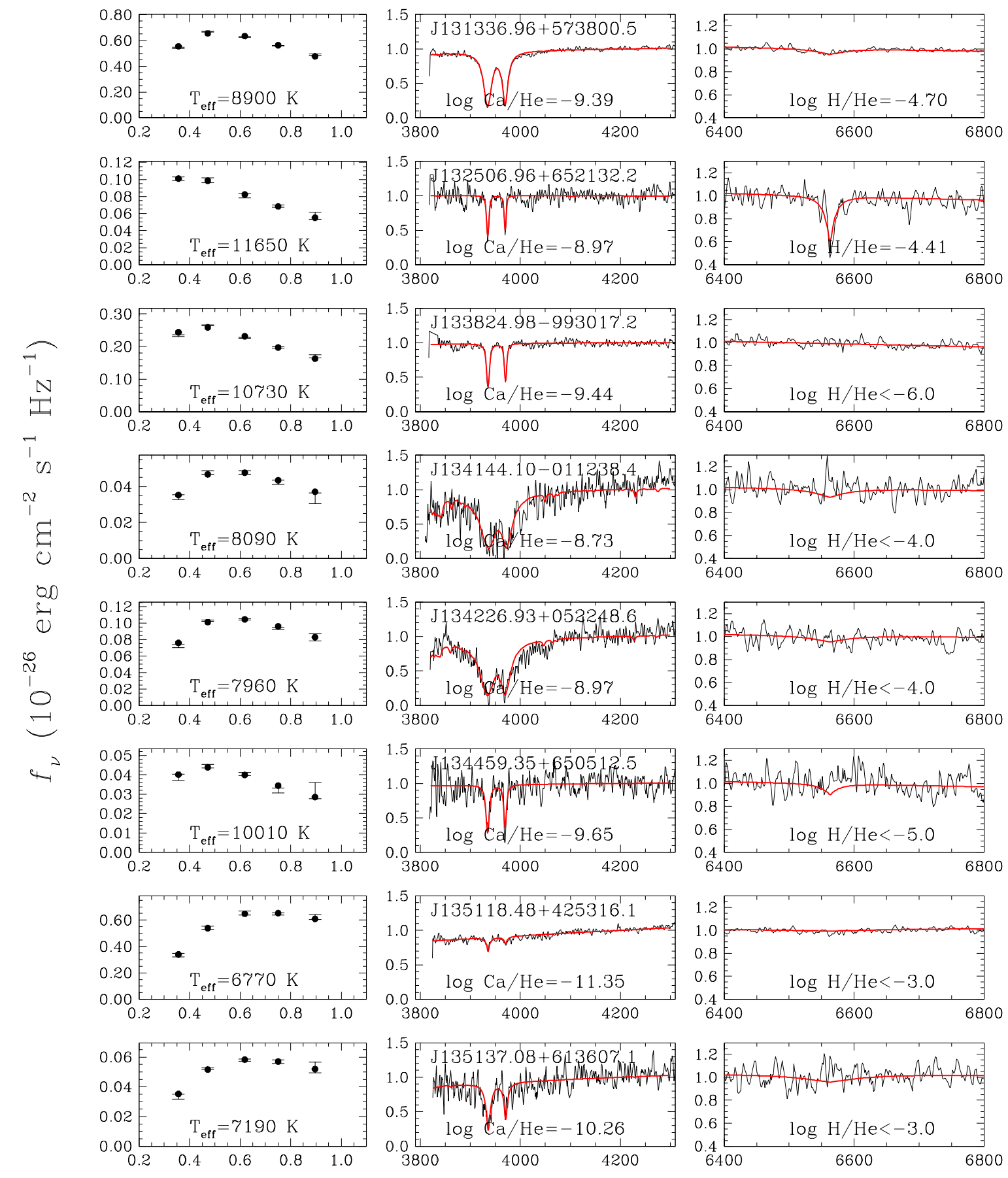

Wavelength ( $\mu \mathrm{m})$
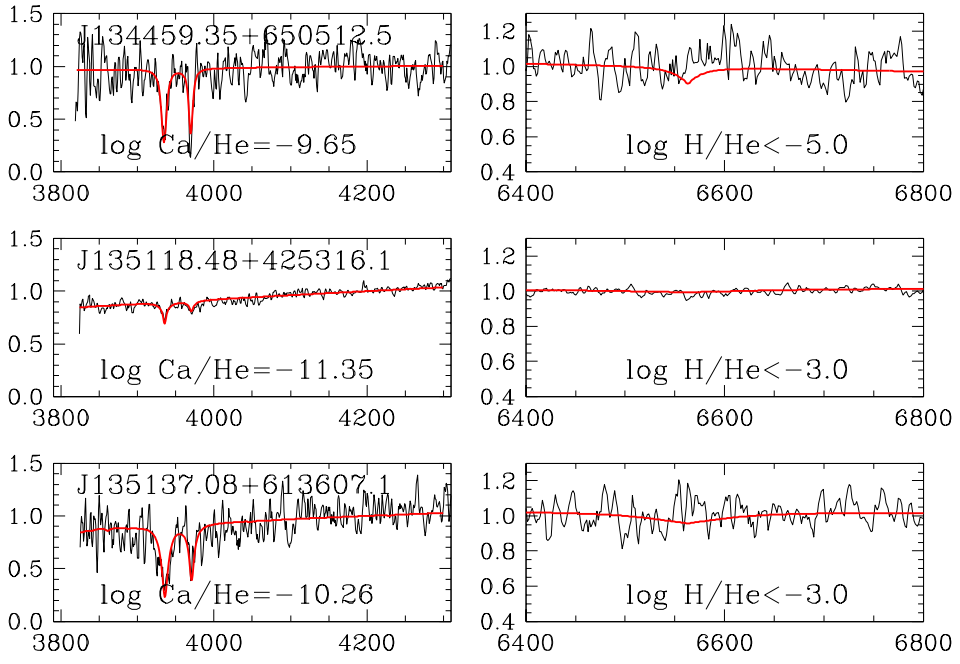

Wavelength $(\AA)$

Figure 19 

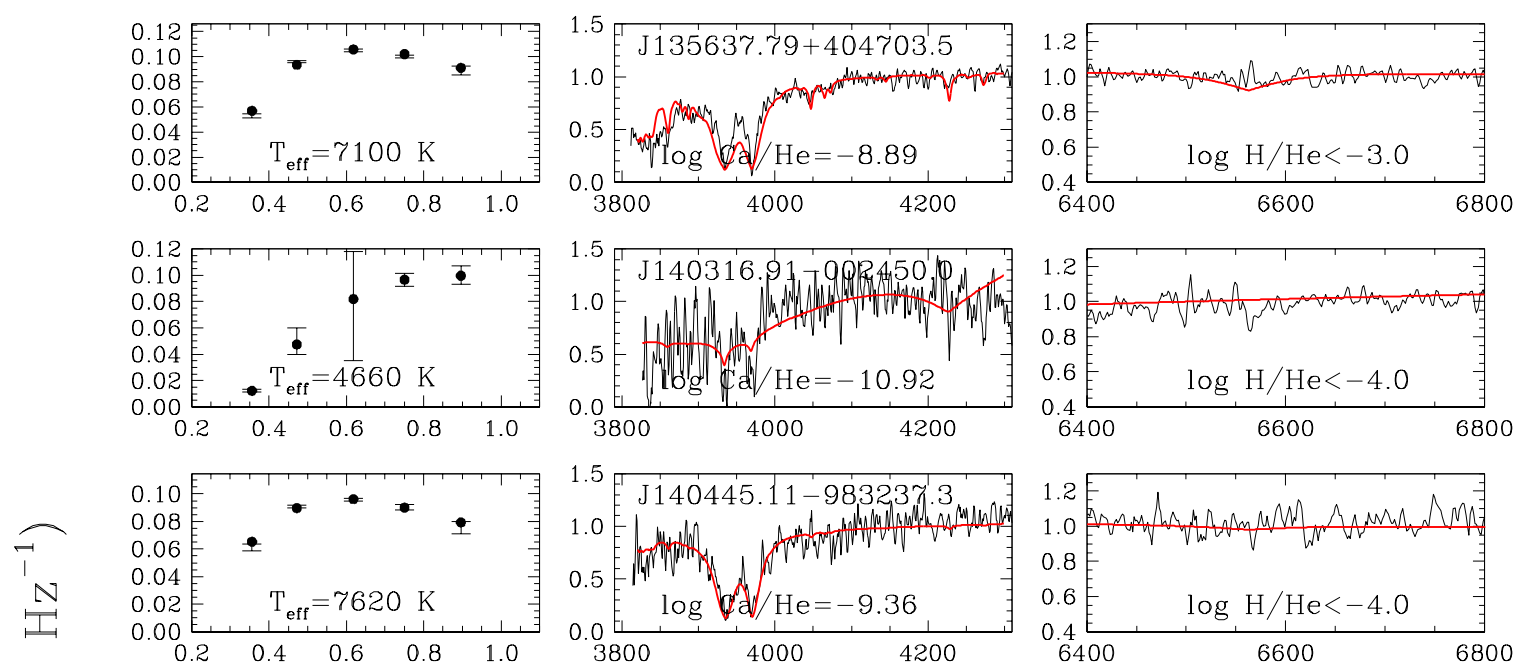

广
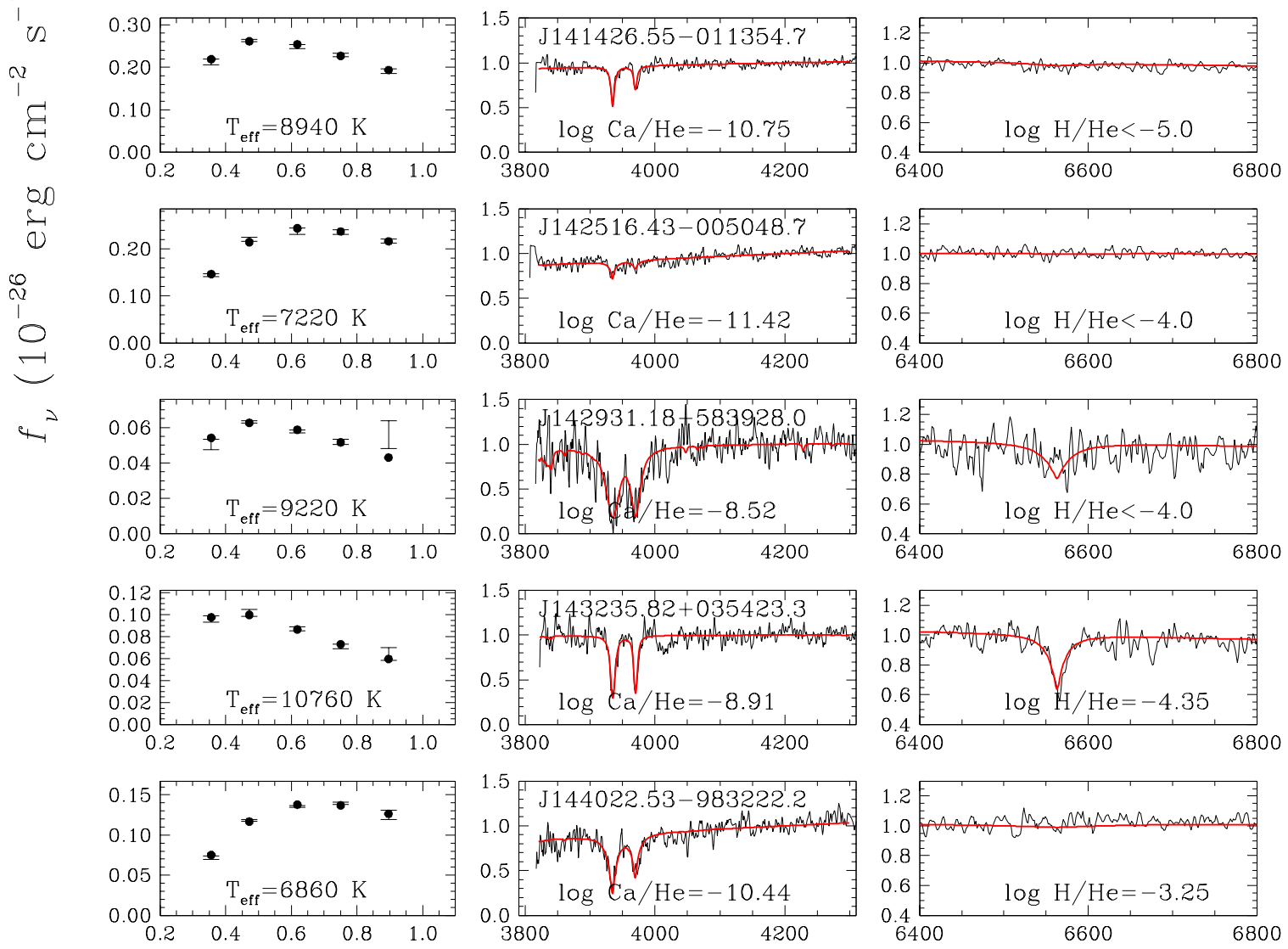

Wavelength $(\mu \mathrm{m})$

Wavelength $(\AA)$ 

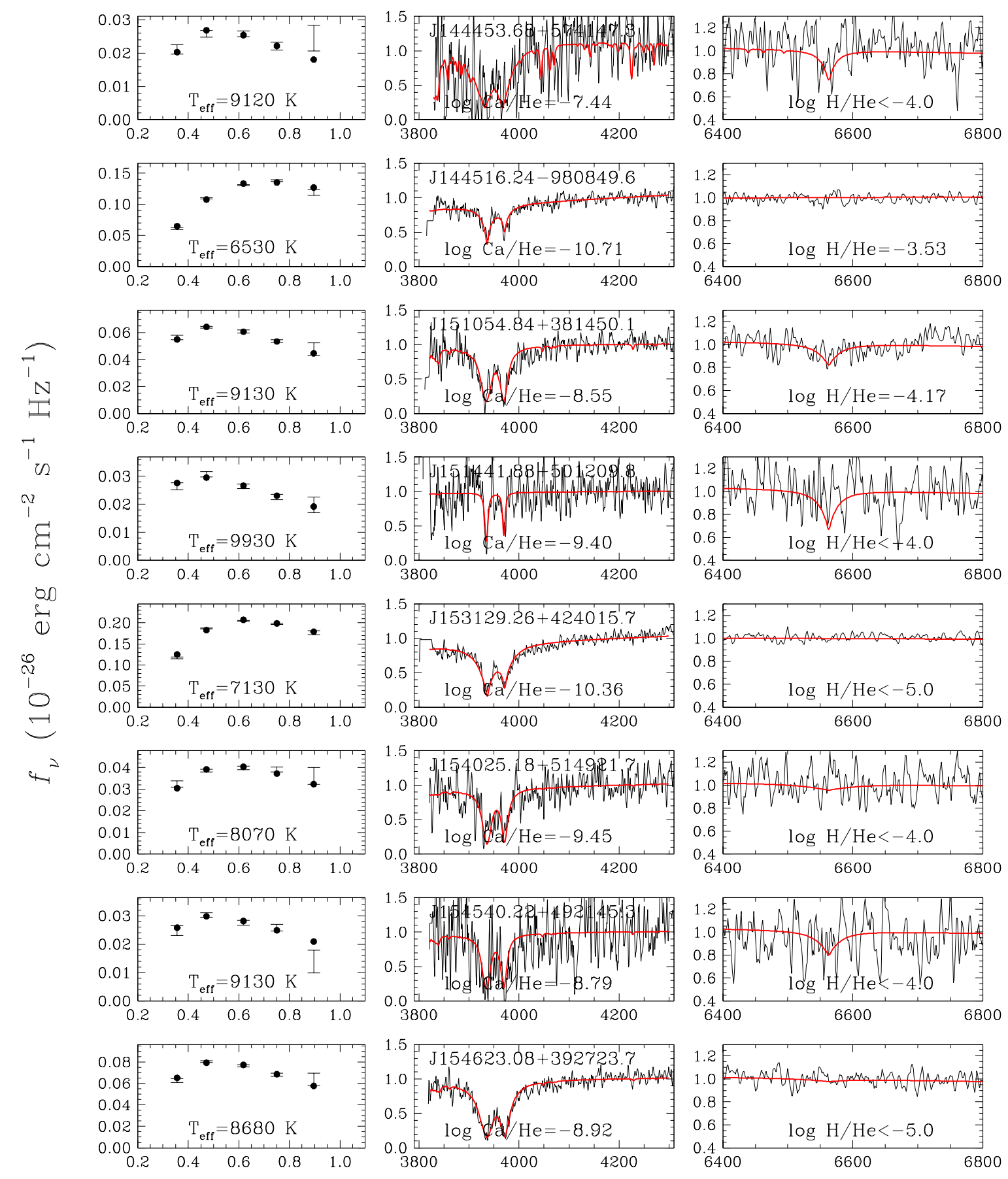

Wavelength ( $\mu \mathrm{m})$
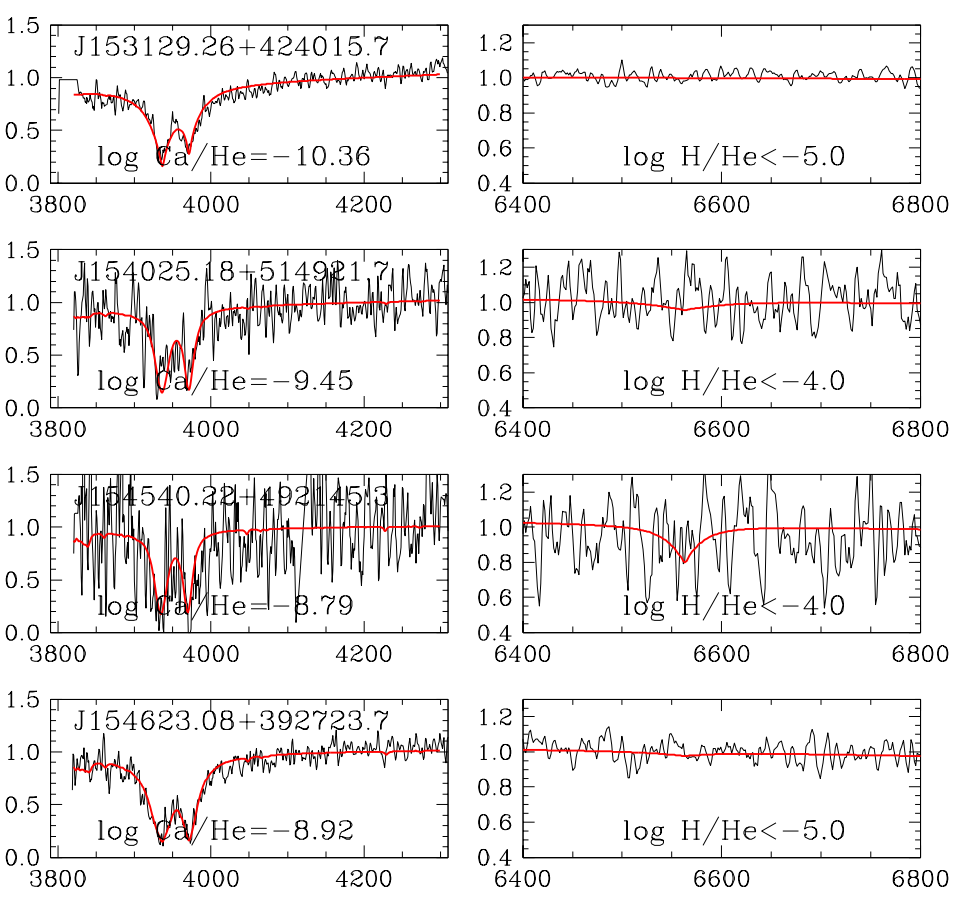

Wavelength $(\AA)$

Figure 21 

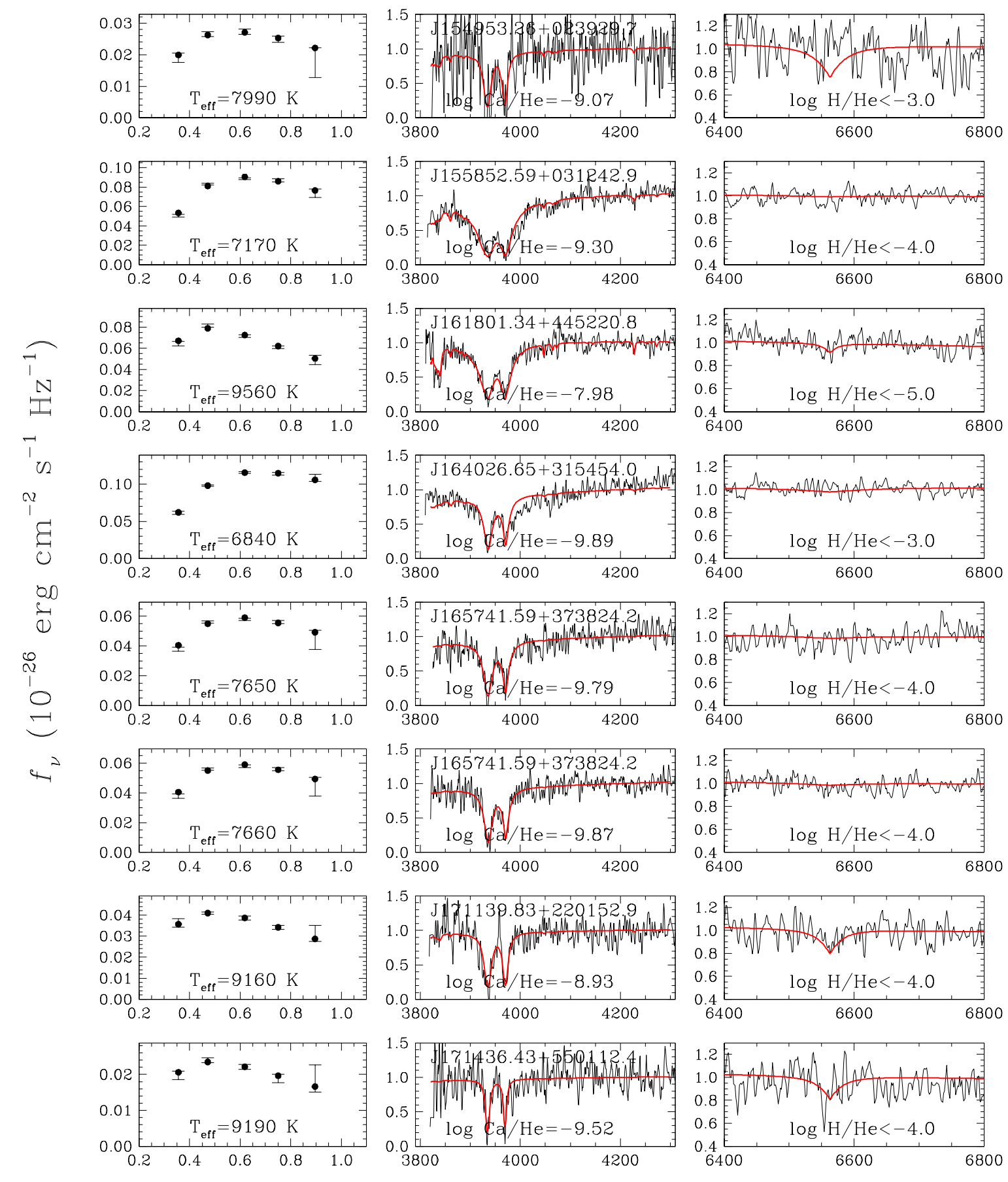

Wavelength ( $\mu \mathrm{m})$
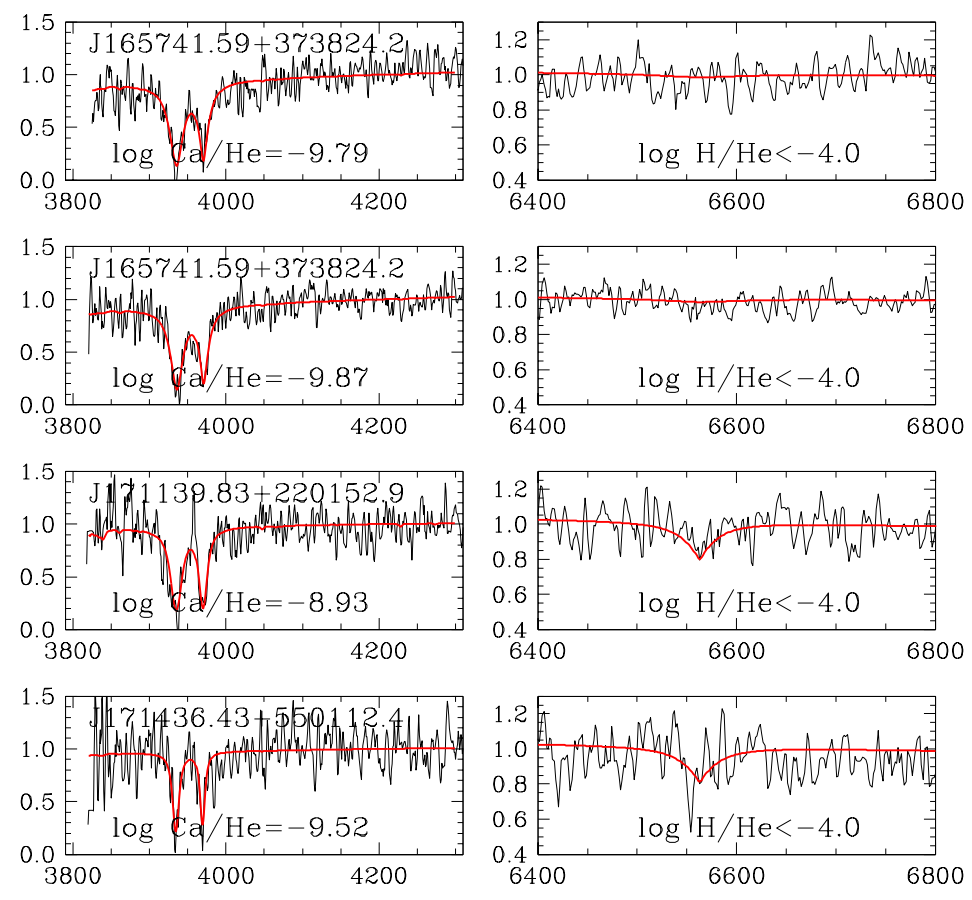

Wavelength $(\AA)$

Figure 22 

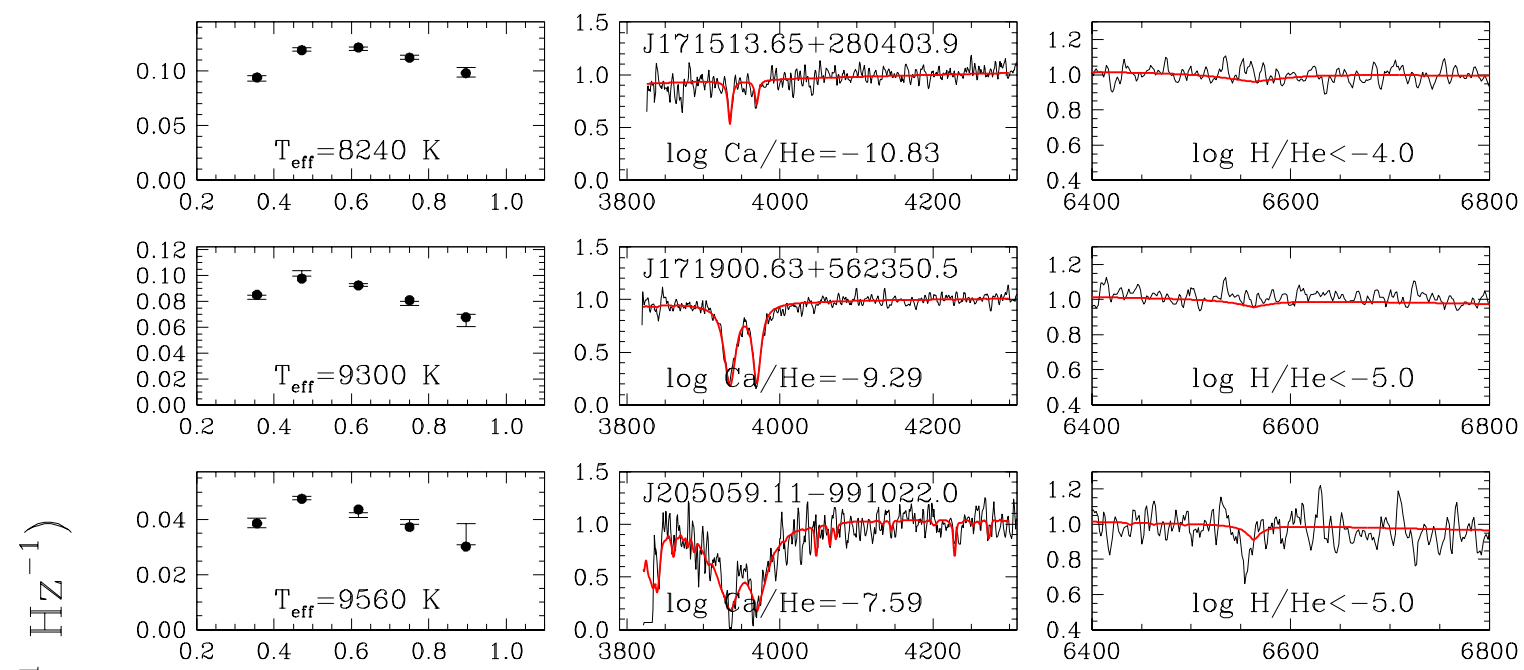

广
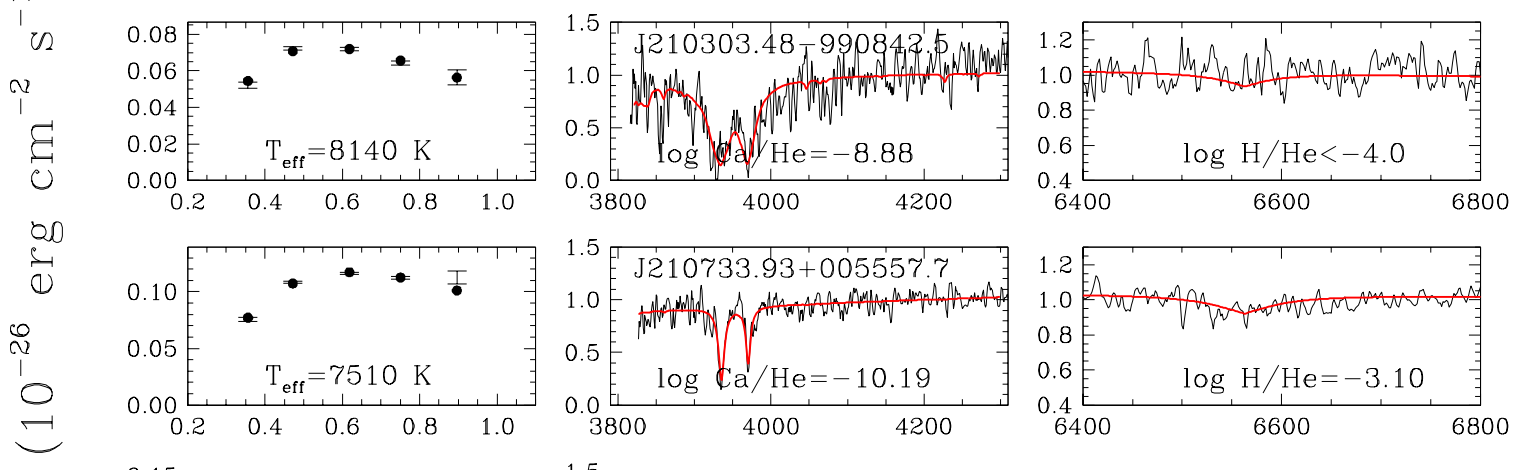

$4^{2}$
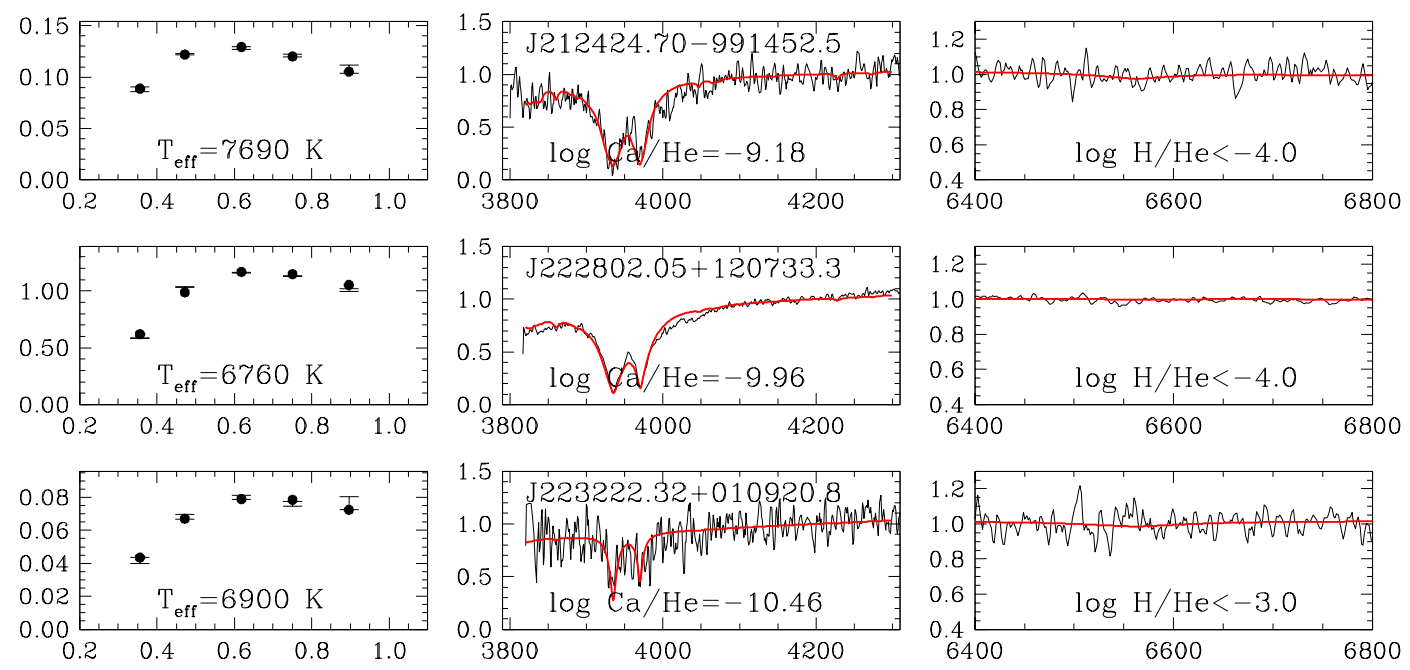

Wavelength $(\mu \mathrm{m})$

Wavelength $(\AA)$

Figure 23 

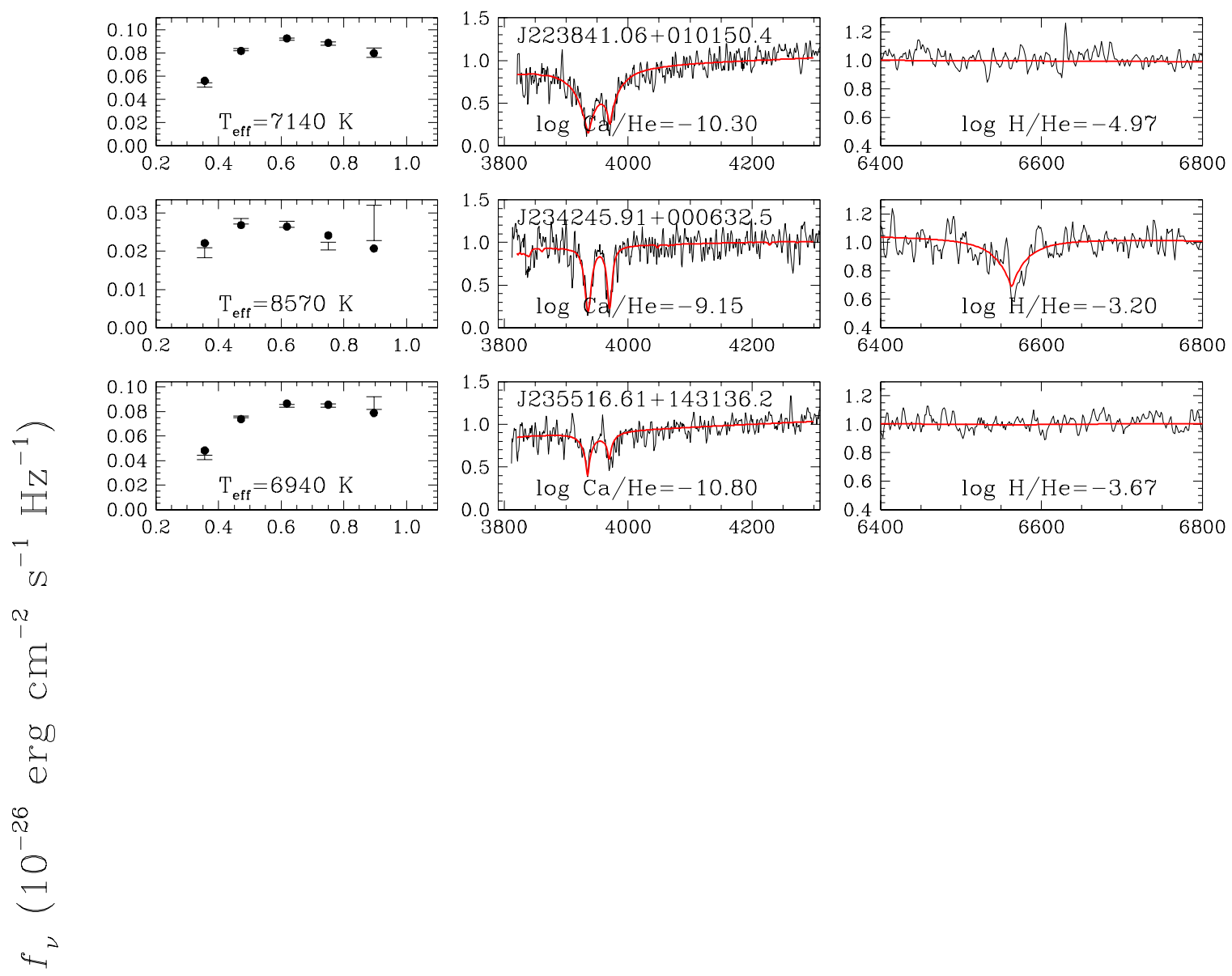

U

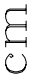

id

$\stackrel{\infty}{\sim}$

E

$4^{2}$

Wavelength $(\mu \mathrm{m})$

Wavelength $(\AA)$

Figure 24 


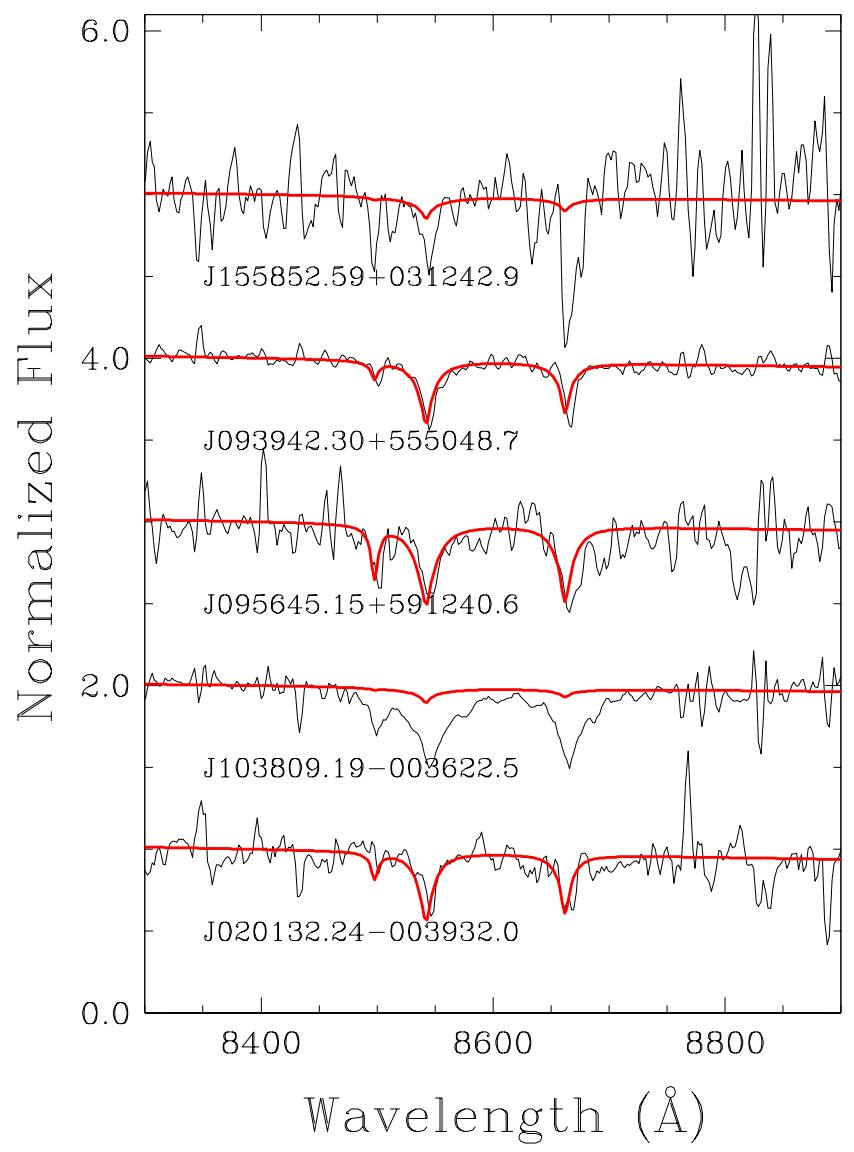

Figure 25 


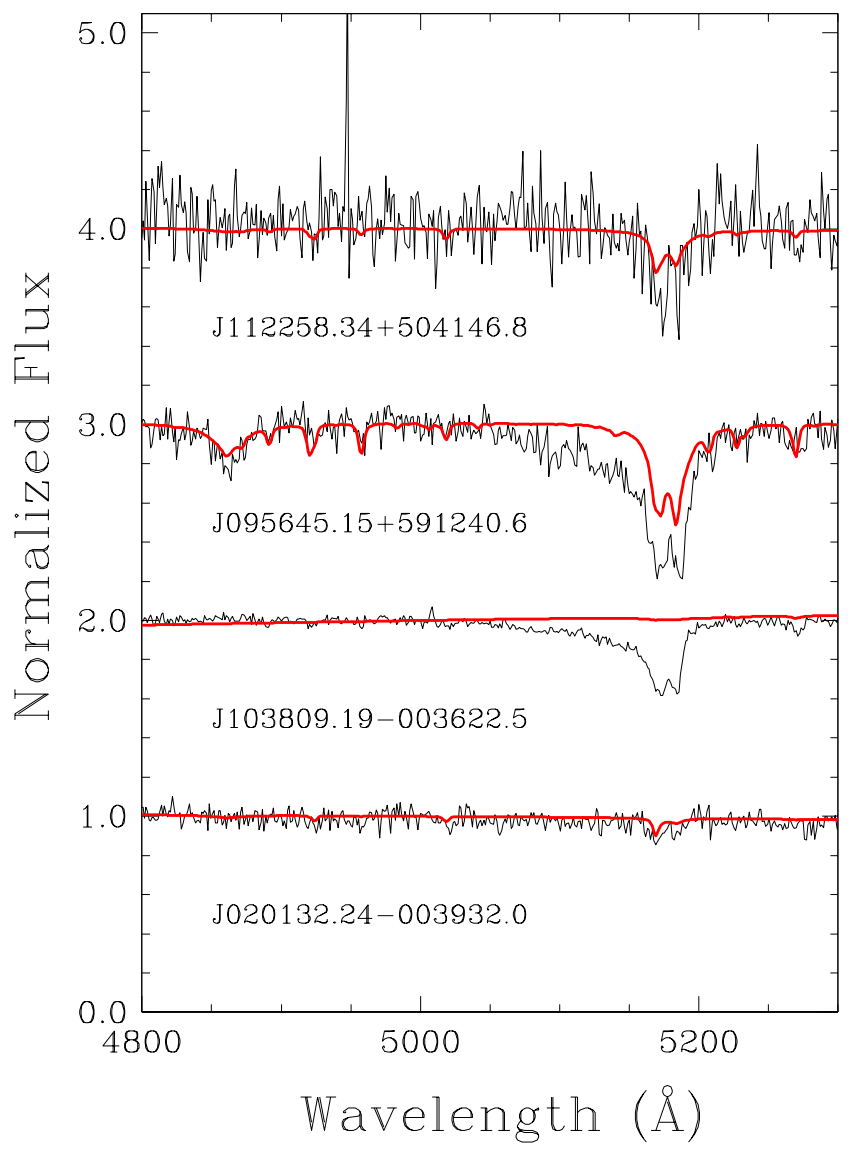

Figure 26 


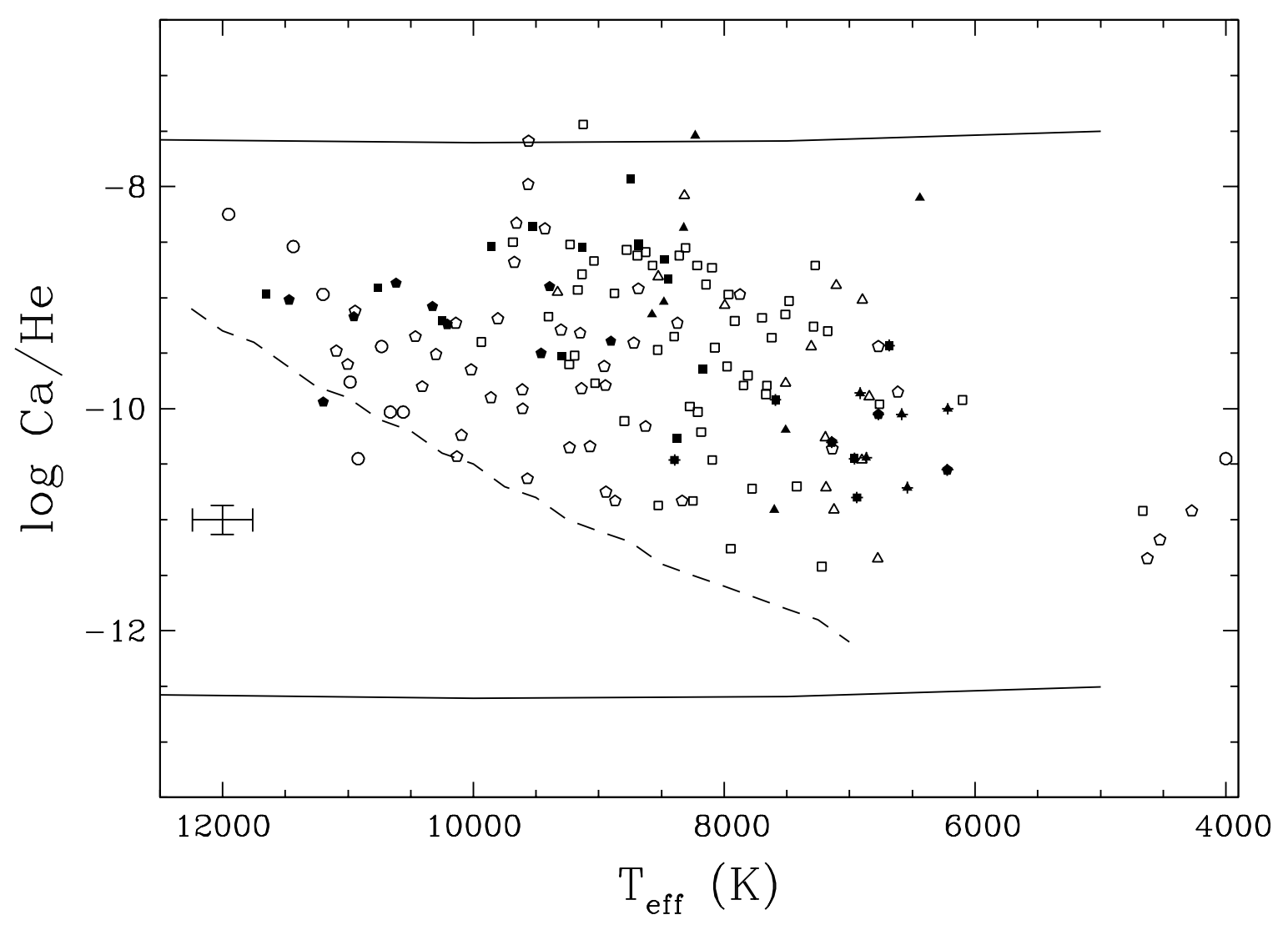

Figure 27 


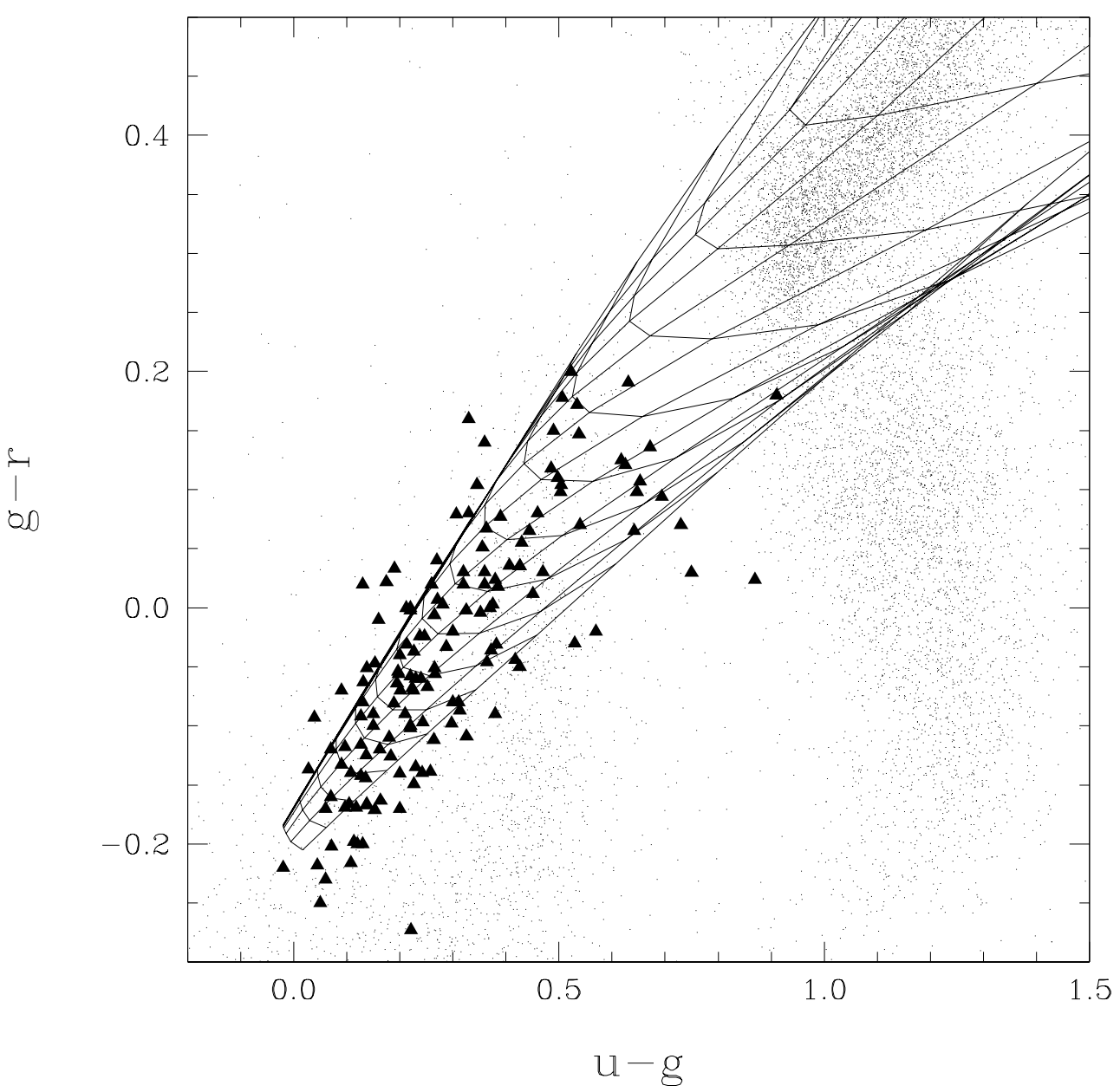

Figure 28 


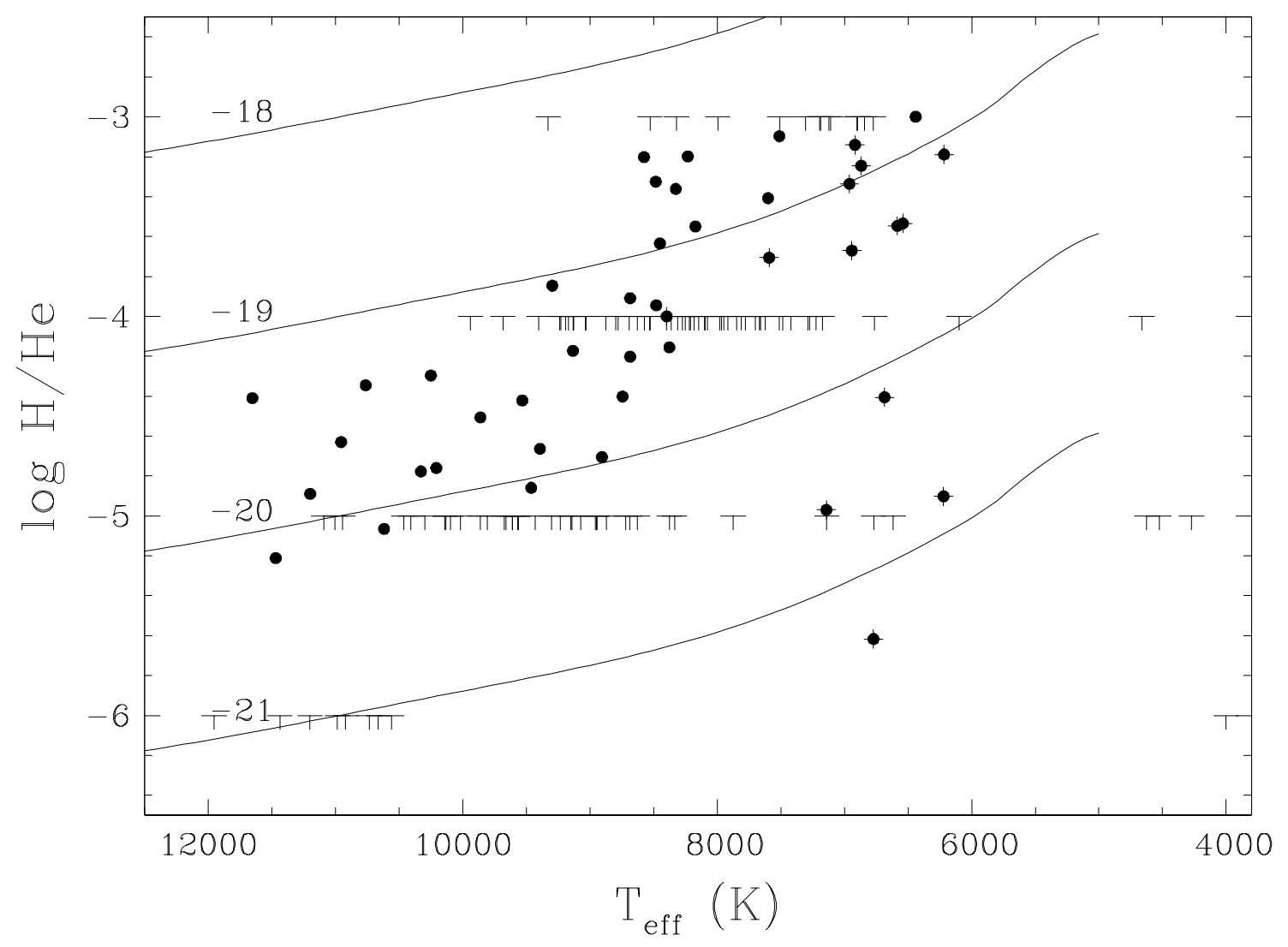

Figure 29 


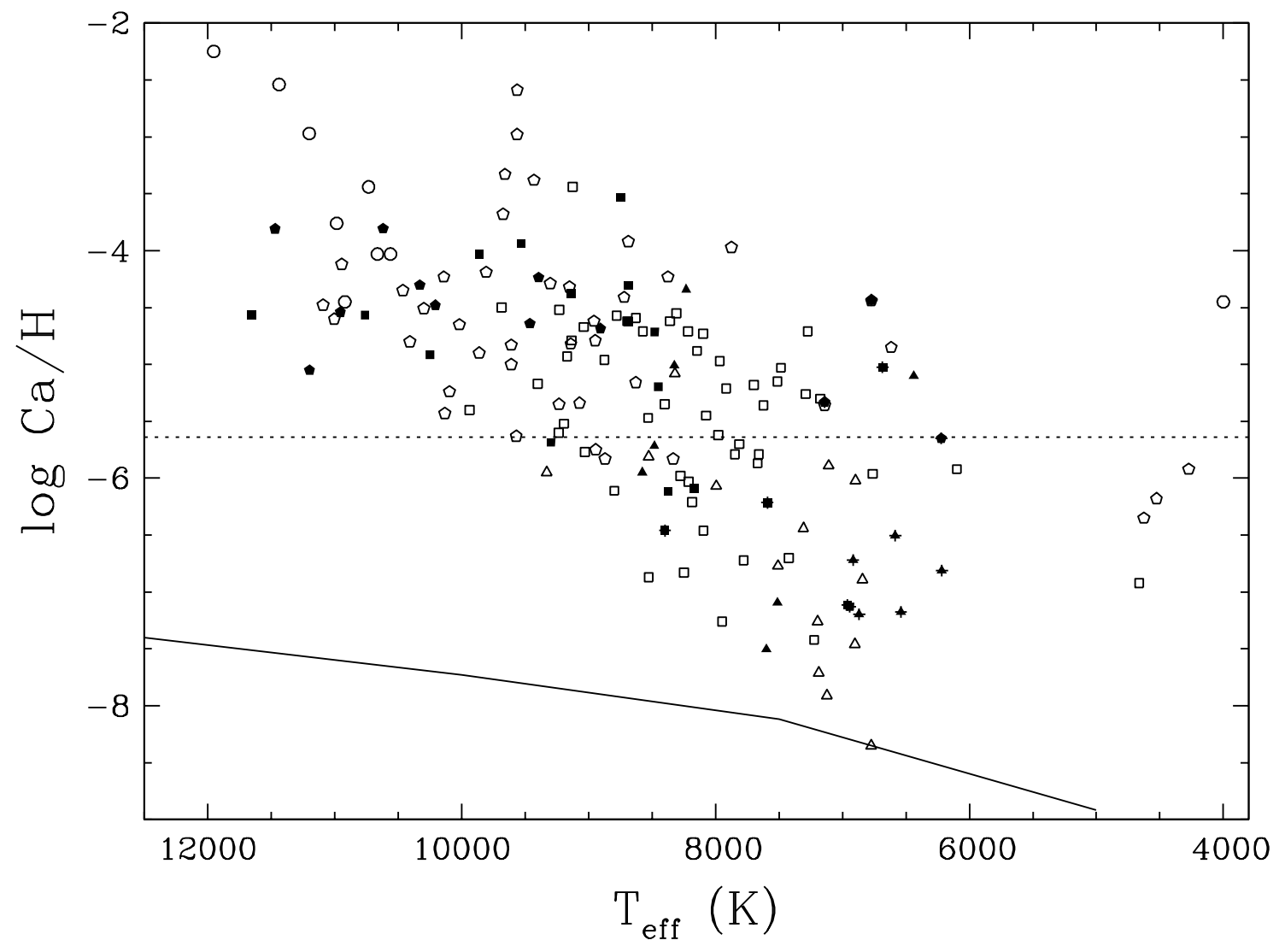

Figure 30 


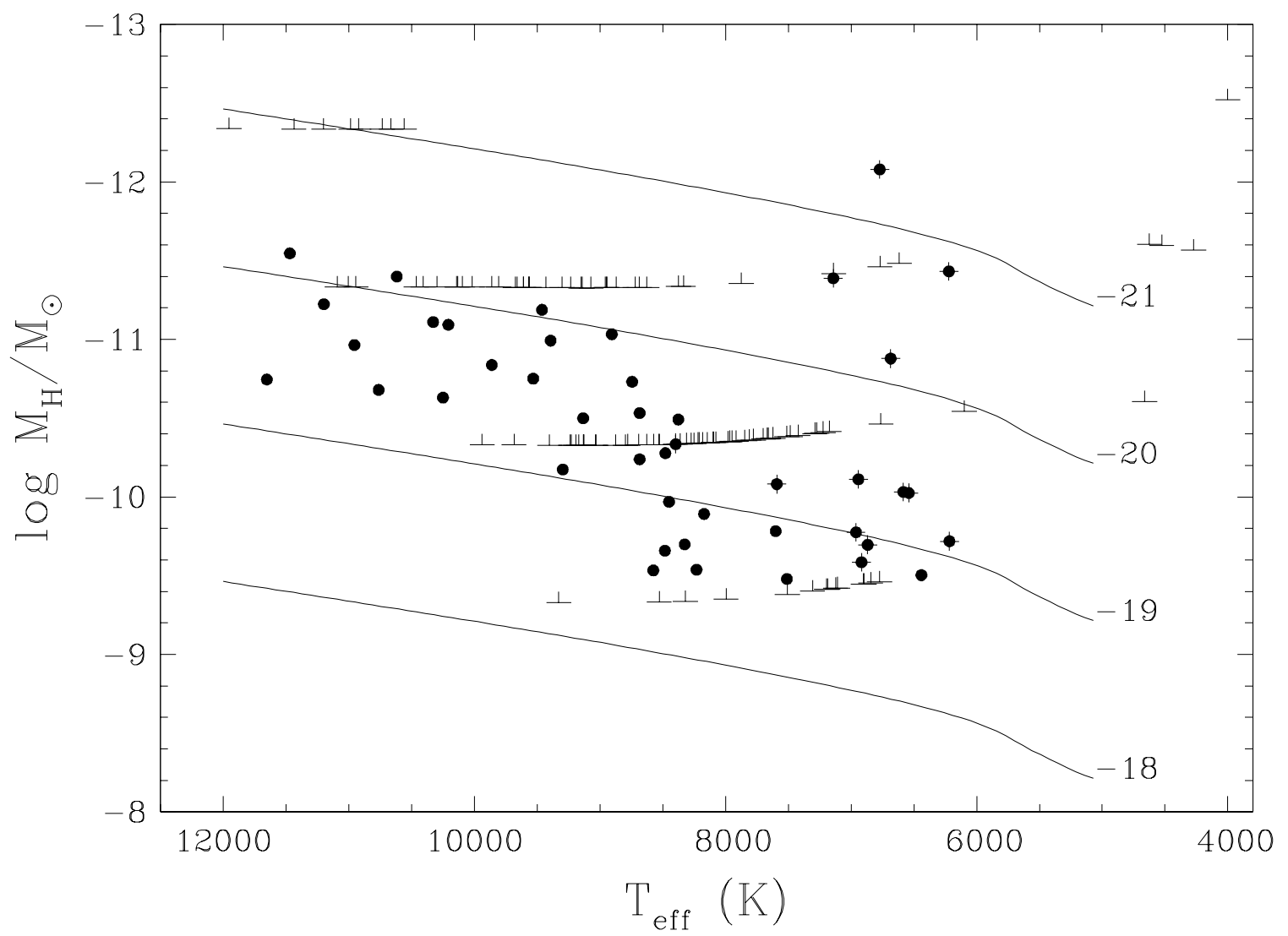

Figure 31 


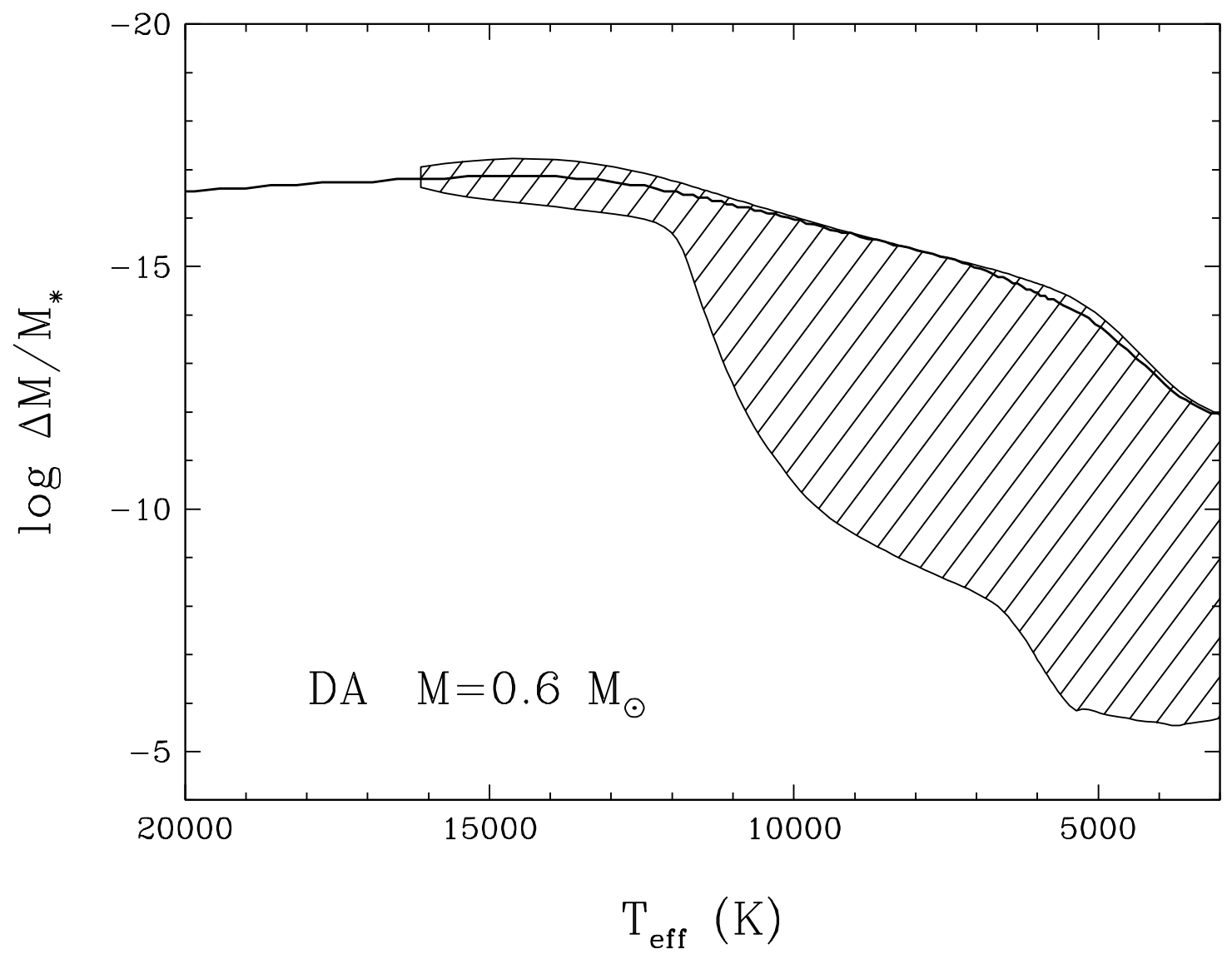

Figure 32 